This document is downloaded from DR-NTU (https://dr.ntu.edu.sg) Nanyang Technological University, Singapore.

Preparation of plasmonic gold nanoparticles and their bio-photonics applications

Gong, Tianxun

2015

https://hdl.handle.net/10356/65565

https://doi.org/10.32657/10356/65565 


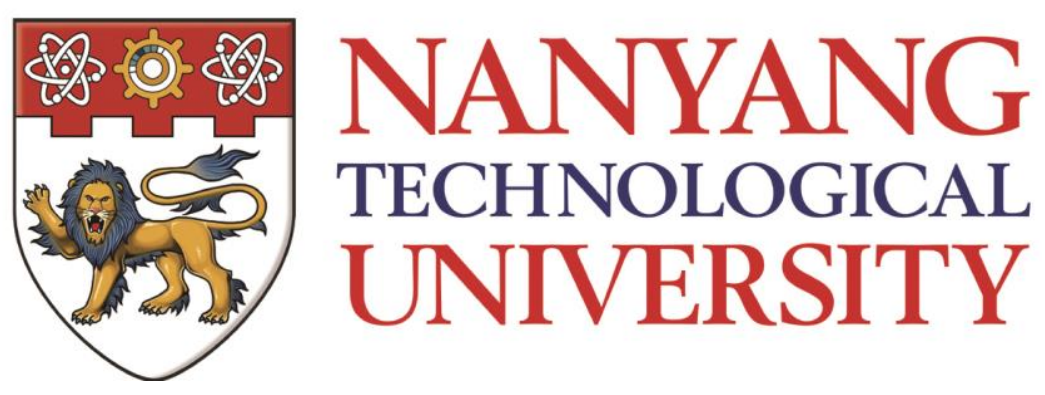

PREPARATION OF PLASMONIC GOLD

NANOPARTICLES AND THEIR BIO-PHOTONICS

APPLICATIONS

TIANXUN GONG

SCHOOL OF ELECTRICAL AND ELECTRONIC ENGINEERING 2015 



\section{PREPARATION OF PLASMONIC GOLD NANOPARTICLES AND THEIR BIO-PHOTONICS APPLICATIONS}

\section{TIANXUN GONG}

School of Electrical and Electronic Engineering

A thesis submitted to the Nanyang Technological University in partial fulfilment of the requirement for the degree of

Doctor of Philosophy 



\section{Acknowledgement}

It would not have been possible for me to complete my Ph.D. study without the guidance, encouragement, support and understanding from many people. I would like to take this opportunity to express my sincere appreciation to all of them.

Firstly and foremost, I would like to express my deepest appreciation to my supervisor Prof. Yong Ken-Tye for his inspiring guidance, great patience and unfailing encouragement. I have benefited a lot from his enthusiasm, and insightful views both on research and life.

I also like to thank Dr. Malini Olivo, head of Bio-optical Imaging Group, SBIC, A*STAR for offering me the opportunity to work with her team on these innovative multidiscipline research projects. I am also grateful to the people in her group, Dr. Dinish U.S., Dr. Kien Voon Kong, Dr. Perumal Jayakumar, Dr. Ghayathri Balasundaram, Dr. Ho Jun Hui, Dr. Amalina Binte Ebrahim Attia, Dr. Wong Chi Lok and Mr. Douglas Goh, for their valuable comments and constructive suggestions during my research.

I owe my gratitude to Prof. Shum Ping and Prof. Luan Feng for their inspiration guidance and support during my early days of research. It has been a great pleasure and honor to work with their innovative research groups. My thanks also go to my friends and previous colleagues in Network Technology 
Research Centre, Wang Guanghui, Hu Juanjuan, Yu Xia, Ouyang Chunmei, Jiang Meng, Liu Huanhuan, Wu Kan, Wu Xuan, Zu Peng, Zhou Junqiang, Huang Ying, Poh Khoon Yong, Chong Lui Tai, Xiong Fei, Cui Ying, Xiao Zhe, Jiang Kai, Karolina Milenko, Zhang Yixin, Yu Xiaojun, Wang Dawei, Li Yihui and all other members. I had a fruitful time with them.

I would also like to express my gratitude to Prof. Georges Humbert from Xlim France, Dr. Hu Rui, Zeng Shuwen, Wang Yucheng, Zhang Butian, Song Peiyi, Tng Jian Hang from NTU Photonic Lab I, for their valuable discussions and suggestions.

I am particularly in debt to my family especially my parents. It is their lasting love, encouraging and support all these years that saw me through tough times and achieved so far in my life.

Finally, I would like to extend my thanks to all the people who have helped me these years. 


\section{Table of Contents}

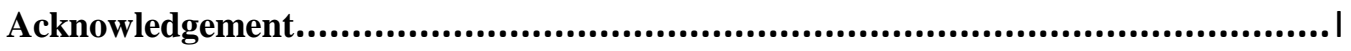

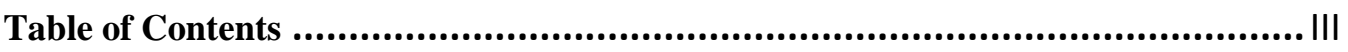

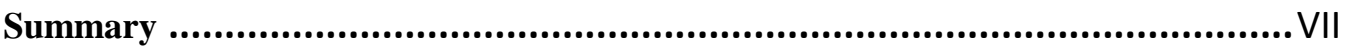

List of Abbreviations ............................................................................... XII

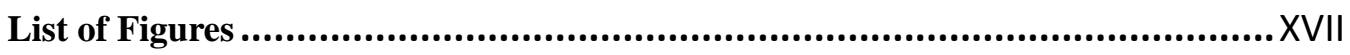

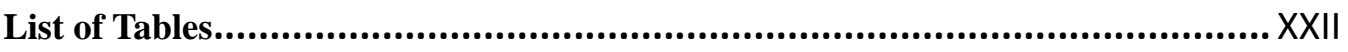

Chapter 1 Introduction ...................................................................... 1

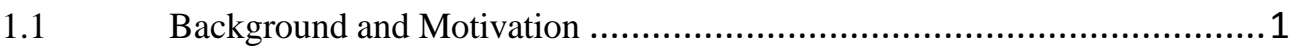

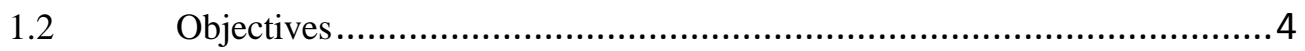

$1.3 \quad$ Major Contributions of The Thesis .............................................. 4

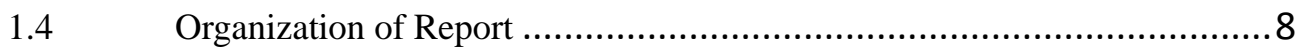

Chapter $2 \quad$ Literature Review.............................................................. 11

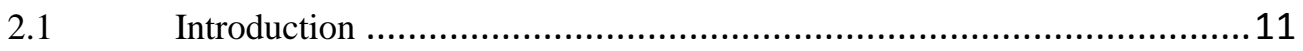

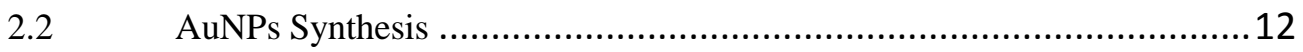

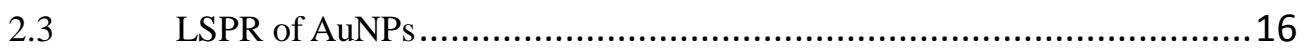

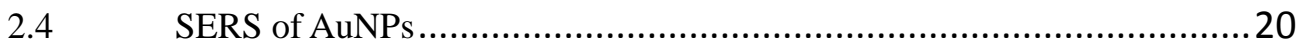

2.4.1 Substrate-Based SERS …......................................................22

2.4.2 Fiber-Based SERS ...................................................................24

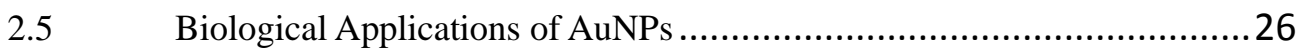

Chapter 3 In Vitro Toxicity Studies of AuNRs formulations Coated with

Bio-functional Thiol-PEG Molecules and Pluronic Block Copolymers................... 31

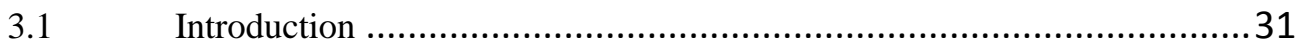

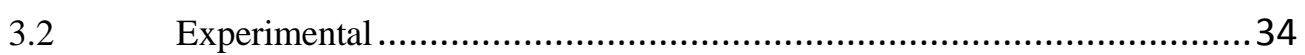

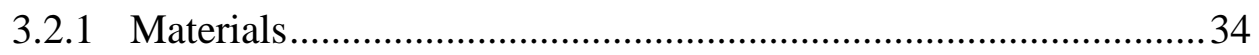

3.2.2 AuNRs synthesis and characterization.........................................34

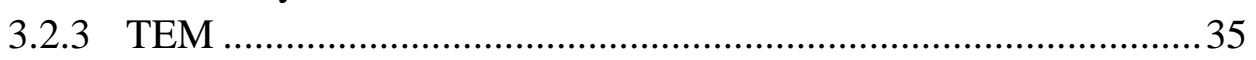

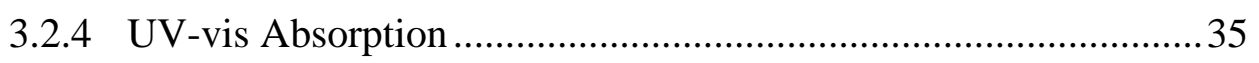

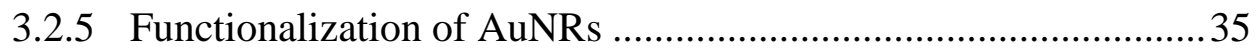

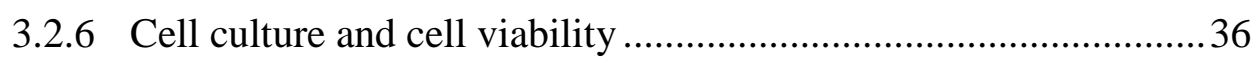

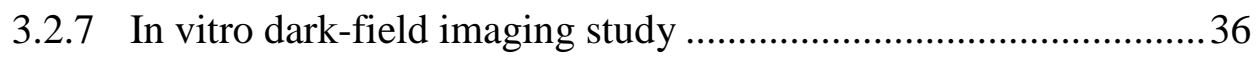

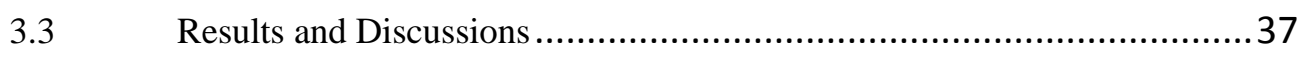

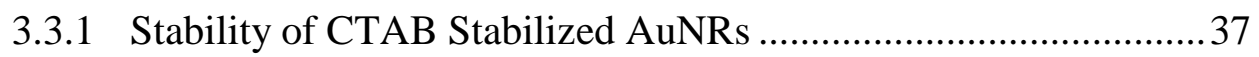

3.3.2 PEG-SH or Pluronic Stabilized AuNRs .........................................39

3.3.3 Cell Viability ......................................................................... 41

3.3.4 Mean Hydrodynamic Diameters of AuNRs ..................................44

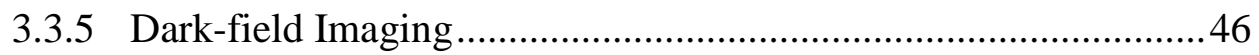

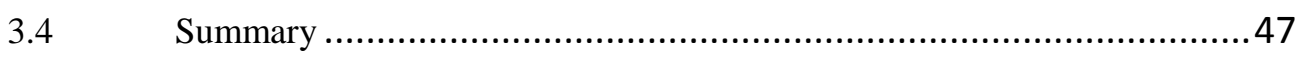

Chapter 4 Biocompatible Probes Based on Pluronic Triblock Copolymer

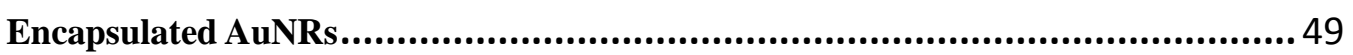

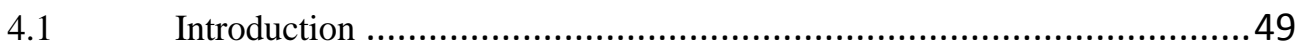




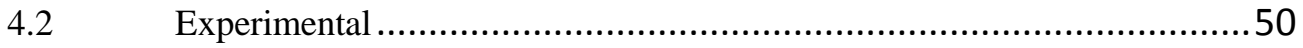

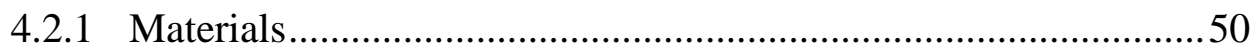

4.2.2 Gold Nanoparticle Seed Synthesis ............................................51

4.2.3 Encapsulation of AuNRs with Pluronic ......................................51

4.2.4 Dark-field Imaging ............................................................52

4.2.5 Differential Interference Contrast Microscopy …..........................52

4.2.6 Other Experiments ....................................................................52

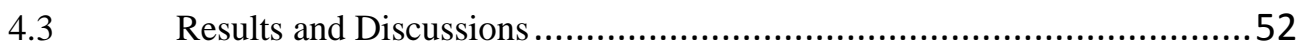

4.3.1 AuNRs Synthesis and Encapsulation .........................................52

4.3.2 Cell viability and DIC microscopy ..........................................56

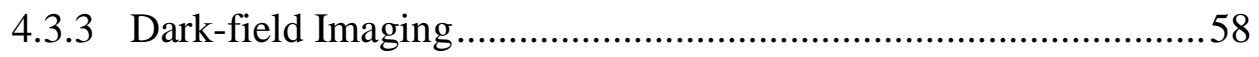

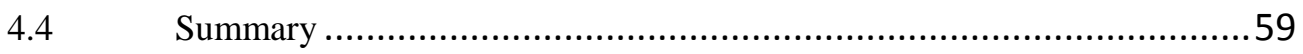

Chapter 5 Bioconjugated AuNSs and AuNRs as Label-free Plasmon Scattering

Probes for Ultrasensitive Multiplex Dark-field Imaging of Cancer Cells ................ 61

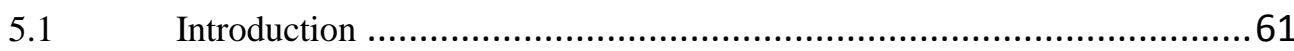

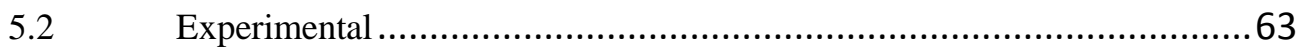

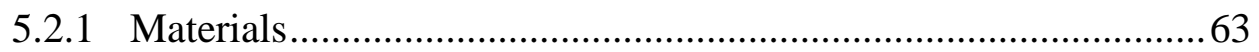

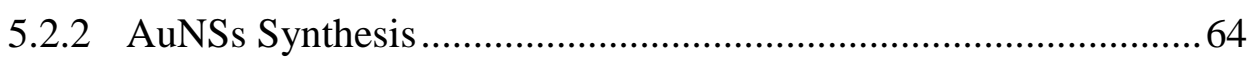

5.2.3 PEG functionalization of AuNRs and AuNSs .............................64

5.2.4 Anti-EGFR Antibody Conjugation of AuNRs and AuNSs...........64

5.2.5 Dynamic Light Scattering and $\zeta$-potential ................................6...65

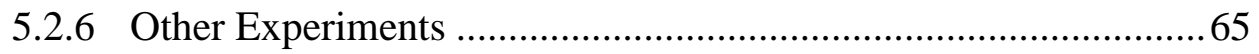

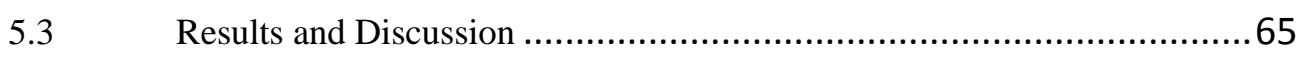

5.3.1 PEGylation of AuNRs and AuNSs .........................................65

5.3.2 Colloidal Stability of PEGylated AuNSs and AuNRs ..................68

5.3.3 Cell viability of PEGylated AuNSs and AuNRs ..........................69

5.3.4 Conjugated AuNRs and AuNSs for Dark-field Multiplex Imaging of Cancer Cells and Its Potential Applications ............................. 71

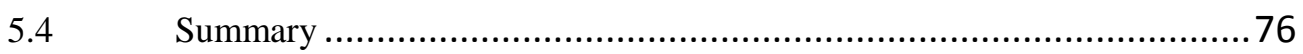

Chapter 6 Optimized Sandwiched Surface Plasmon Resonance Enhanced

Bio-sensor for Multiplex Biomarker Detection............................................... 77

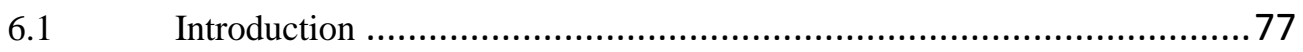

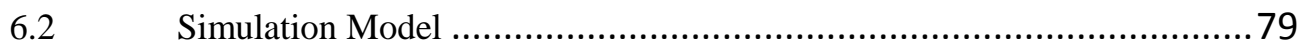

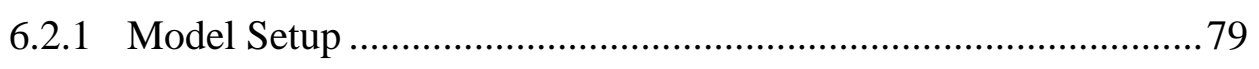

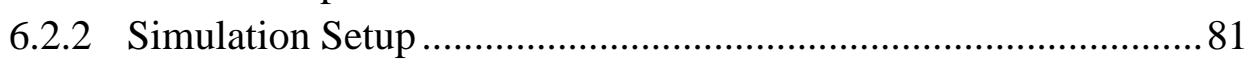

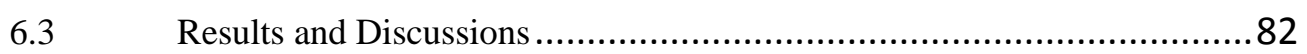

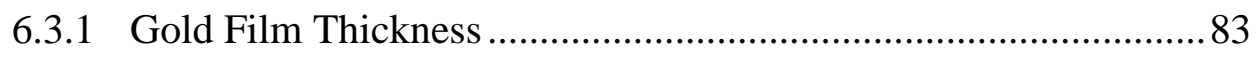

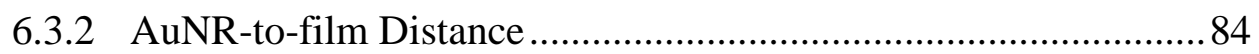

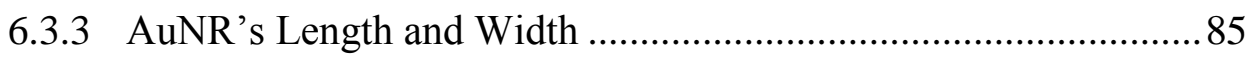

6.3.4 Bio-sensor Concept for Multiplex IgG Proteins Detection ...........86

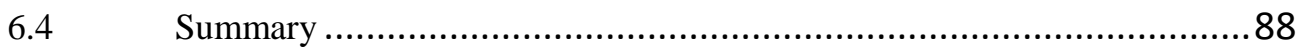

Chapter 7 Highly Sensitive SERS Detection and Quantification of Sialic Acid on

Single Cell Using Photonic-Crystal Fiber with AuNPs...................................... 89

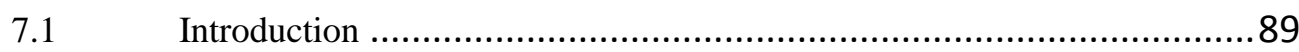




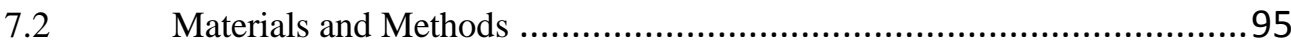

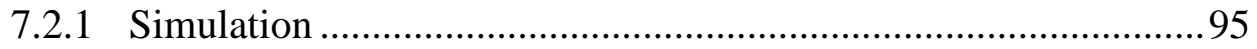

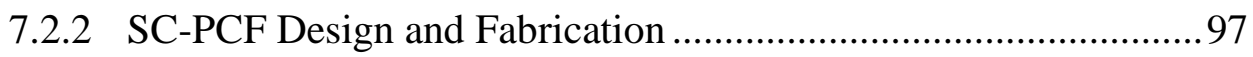

7.2.3 Fiber Preparation for SERS Detection .......................................97

7.2.4 HeLa Cell Culture and Varying Sialic Acid Levels ......................98

7.2.5 SERS Sample Preparation ......................................................... 99

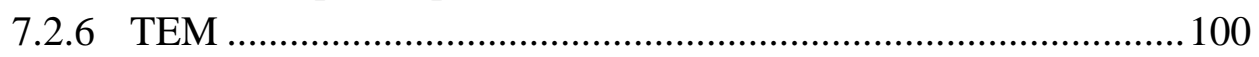

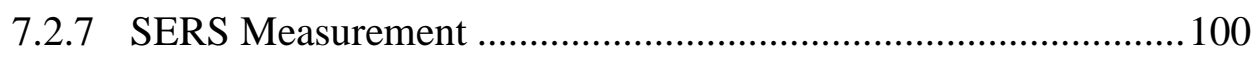

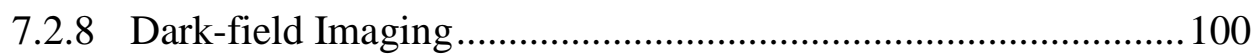

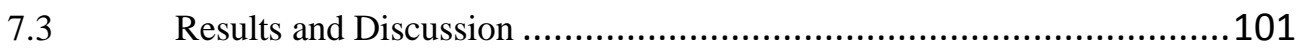

7.3.1 Size Dependence of AuNP on SERS enhancement of DBA ...... 101

7.3.2 SERS Enhancement Comparison Between SC-PCF and Conventional HC-PCF ........................................................... 103

7.3.3 Correlation of DBA Concentration with SERS Intensity ........... 104

7.4 Detection and Quantification of Sialic Acid on Single Cell ....................106

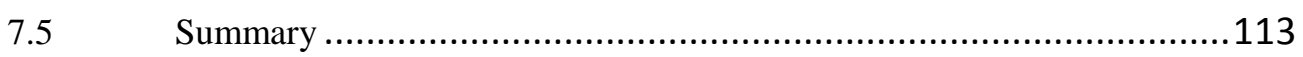

Chapter 8 Sensitive SERS Multiplexed Detection of Matrix Metalloproteinase 2

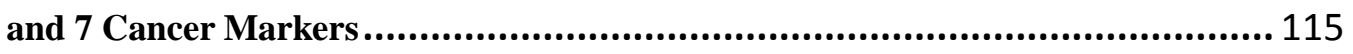

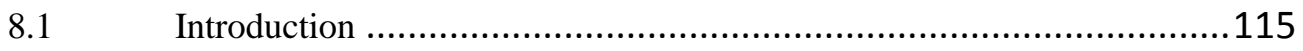

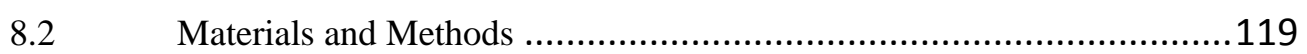

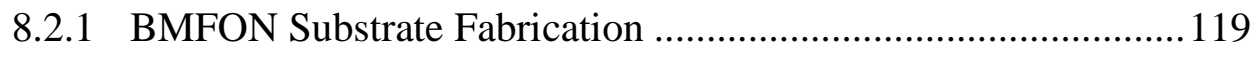

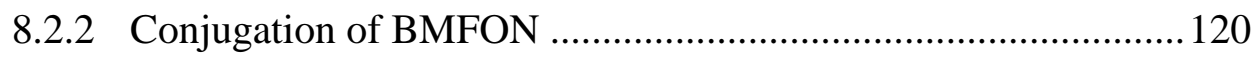

8.2.3 AuNPs Preparation and Characterization.................................... 121

8.2.4 Detection of Individual MMP Enzyme ...................................... 122

8.2.5 SEM Sample Preparation ....................................................... 123

8.2.6 Multiplexed Detection of MMP-2 and MMP-7 enzymes ........... 123

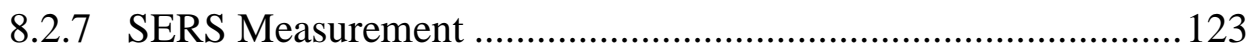

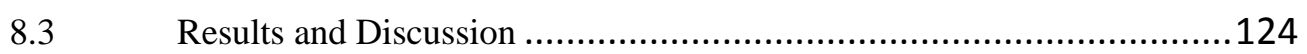

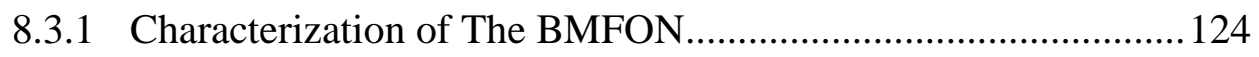

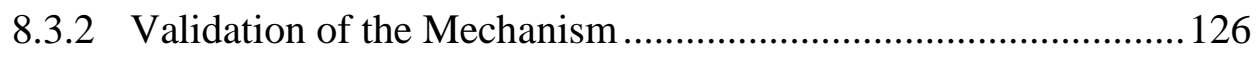

8.3.3 Multiplexed Detection of MMP-2 and MMP-7 Enzymes........... 129

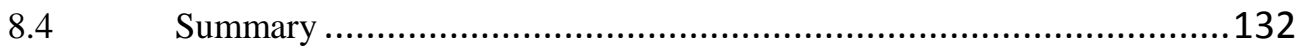

Chapter 9 Conclusion and Future Works ............................................ 135

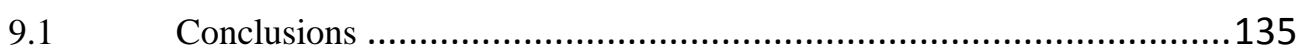

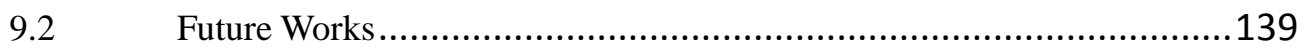

9.2.1 Tunable AuNPs Enhanced SERS Platform................................. 139

List of Publications ........................................................................... 143

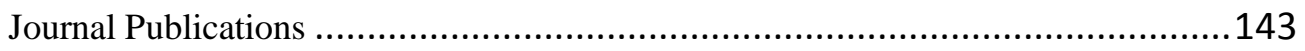

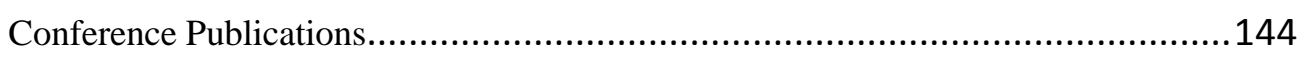

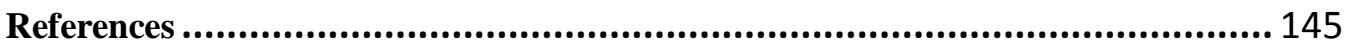




\section{Summary}

Metallic nanoparticles (e.g., gold nanospheres (AuNSs) and gold nanorods (AuNRs)) display the unique feature of localized surface plasmon resonance (LSPR). This feature occurs when the incident photon frequency is resonant with the collective oscillation of the conduction-band electrons of the nanoparticles, giving them remarkable optical absorption and scattering properties. LSPR is highly sensitive to the size and shape of gold nanoparticles (AuNPs) and the external medium. These peculiar features make them useful for bio-imaging and bio-sensing applications. In this thesis, we investigated the optical properties and biocompatibility of AuNPs formulations with different shapes, sizes, and functionalization. We further used AuNPs to develop surface plasmon resonance (SPR)/surface enhanced Raman spectroscopy (SERS) based platforms for bio-imaging and bio-sensing applications. The successful development of such platforms will help doctors and surgeons to better screen and identify cancer and other diseases at an early stage.

To prepare and characterize AuNPs, we investigated the cytotoxicity, colloidal stability, and optical properties of AuNPs before and after functionalizing them with thiolated polyethylene glycol (PEG-SH) and Pluronic triblock copolymer (PEO-PPO-PEO) molecules. The morphology of the functionalized AuNPs was characterized using UV-vis absorption 
spectroscopy, transmission electron microscopy (TEM), and dynamic light scattering (DLS). Solution phase synthesis of AuNRs is the preferred approach for obtaining different sizes and aspect ratios of rod nanoparticles. The use of PEG-SH and PEO-PPO-PEO as capping agents to reduce the cytotoxicity of AuNRs formulations while maintaining their optical, colloidal, and structural properties was investigated. It was found that encapsulating AuNRs with PEG-SH or PEO-PPO-PEO molecules guaranteed the stability and biocompatibility of the nano-formulation. However, the excessive use of these molecules during the passivation process led to a reduction in the overall cell viability. The use of functionalized AuNRs as scattering probes for dark-field imaging of cancer cells demonstrated their good biocompatibility. This study offers a unique solution for the future development of safe scattering color probes for clinical applications such as the long-term imaging of cells and tissues.

For SPR-based bio-imaging and bio-sensing, low toxicity biocompatible block copolymer coated AuNRs were synthesized for cancer cell imaging. In this work, two types of Pluronic triblock copolymers were used for the passivation of AuNRs surface to reduce their cytotoxicity and improve their colloidal and optical stability for biological applications. These plasmonic AuNPs formulations were carefully characterized using UV-vis absorption spectra analysis, TEM, and cell viability studies. Subsequently, the synthesized AuNPs formulations were adopted for cancer-cell imaging using differential 
interference contrast microscopy and dark-field imaging. In addition to Pluronic, the application of PEG-SH functionalized AuNPs as plasmon scattering probes was also investigated for dark-field multiplex imaging of live cancer cells. By carefully engineering the particles' surface, anti-epidermal growth factor receptor (anti-EGFR) antibody conjugated AuNSs and AuNRs dispersions were successfully prepared. The receptor-mediated delivery of antibody conjugated AuNSs and AuNRs into the EGFR receptor-positive oral squamous carcinoma cell (OSCC) line using the dark-field microscopy technique was demonstrated. The results showed that the developed AuNSs and AuNRs formulations could provide up to seven different types of plasmon scattering probes based on their flexible tunability range, with a spectral separation range of $100 \mathrm{~nm}$ within the absorption band.

Lastly, finite element method simulations were used to optimize and understand the configuration of an AuNRs-enhanced SPR sensor and examine the developed system for multiplex antigens detection applications. The near-field coupling between the sensing film and AuNRs was analyzed. By systematically tailoring the simulation parameters of gold film thickness, AuNRs-to-film distance, and AuNRs dimensions on the SPR substrate, it was found that AuNRs with width smaller than $40 \mathrm{~nm}$ caused a significant LSPR wavelength shift of the sensor. This discovery led us to design a new SPR sensing platform for biomolecules detection. The simulation approach was used to demonstrate the concept of functionalized gold film and AuNRs 
conjugated with anti-Immunoglobulin $\mathrm{G}$ antibodies for multiplex detection of various IgG proteins. The SPR sensors adopted AuNRs with three aspect ratios to detect different types of IgG proteins based on different SPR peaks.

For the application of AuNPs to surface enhanced Raman spectroscopy (SERS) bio-sensing, both optical fiber-based and nano-substrate-based platforms were developed. For the fiber-based platform, an ultrasensitive sensing platform which was developed to detect the mean sialic acid level on the surface of a single cell with a sensitivity as low as 2 femto moles. This platform adopted the use of an interference-free Raman tag, 4-(dihydroxyborophenyl) acetylene (DBA), which selectively binds to sialic acid on the cell membrane. By loading the side channel of a photonic crystal fiber with a mixture of AuNPs and DBA-tagged HeLa cells, and subsequently propagating laser light through the central solid core, a strong SERS signal was obtained. Based on this technique, accurate detection and quantification of the sialic acid concentration on single cell was achieved, surpassing previously reported methods that require over $10^{5}$ cells. Moreover, this platform can be developed into a potential clinical diagnostic tool to analyze sialic acid related diseases such as tumor malignancy and metastasis in real-time. For the substrate-based platform, a platform was developed for the sensitive multiplexed detection of matrix metalloproteinases (MMP) (MMP-2 and MMP-7) with a low detection limit and high specificity based on the enzymatic reactions by which a peptide can only be cleaved by its 
corresponding enzyme. The platform comprised two components, a specialized SERS-based bimetallic film over nanosphere (BMFON) substrates and AuNPs. The two components were functionalized such that binding between the two would occur through biotin-avidin-biotin complexation. The binding was hindered by MMP peptide chains that were conjugated onto the surfaces of the substrate and AuNPs, and the bonds could only be removed by cleaving the peptide chains with corresponding enzymes. Because AuNP binding sites become free after peptides are cleaved, the number of binding sites for AuNPs on the substrate increase. By tagging the AuNPs, concentrations of MMP-specific enzymes can be quantified through examining the intensities of signature SERS peaks of the tags. This cleave-and-bind mechanism was first validated by the individual detection and quantification of MMP-2 and MMP-7. The platform was demonstrated to sensitively detect concentrations of specific enzymes ranging from $1 \mathrm{ng} / \mathrm{mL}$ to $40 \mu \mathrm{g} / \mathrm{mL}$. Finally, the multiplexed detection of MMP-2 and MMP-7 was demonstrated. This work can be further developed as a clinical diagnostic tool to detect other MMP proteinases in bio-fluid samples. 


\section{List of Abbreviations}

A

ATP

4-Aminothiophenol

AuNPs

Gold Nanoparticles

AuNRs

Gold Nanorods

AuNSs

Gold Nanospheres

B

BMFON

Bimetallic-Film-Over-Nanosphere

BSA

Bovine Serum Albumin

C

CTAB

Cetylmethylammonium Bromide

D

DBA

4-(Dihydroxyborophenyl) Acetylene

DIC

Differential Interference Contrast

DLS

Dynamic Light Scattering

DMEM

Dulbecco's Modified Eagles's Medium 
DMSO Dimethyl Sulfoxide

$\mathbf{E}$

ECM Extracellular Matrix

EDC Ethyl(dimethylaminopropyl) Carbodiimide

EDTA Ethylenediaminetetraacetic Acid

EGFR Epidermal Growth Factor Receptor

ELISA Enzyme-Linked Immunosorbent Assay

$\mathbf{F}$

FBS

Fetal Bovine Serum

FEM

Finite Element Method

FONs Film Over Nanosphere

FRET Fluorescence Resonance Energy Transfer

$\mathbf{H}$

HC-PCF Hollow Core Photonic Crystal Fiber

I

IgG Immunoglobulin G 
$\mathbf{L}$

LPR

Longitudinal LSPR

LSPR

Localized Surface Plasmon Resonance

M

MMP

Matrix Metalloproteinase

MPA

3-Mercapto-Propionic Acid

$\mathbf{N}$

NHS

N-Hydroxysuccinimide

NT

2-Naphthalenethiol

$\mathbf{O}$

OSCC

Oral Squamous Carcinoma Cells

$\mathbf{P}$

PAA

Polyacrylic Acid

PAH

Poly(allylamine) Hydrochloride

PBA

Phenyl-Boronic Acid

PBS

Phosphate Buffer Sulphate 
PEG-SH

PEI

PEO

PEO-PPO-PEO

PML

PPO

$\mathbf{R}$

RF

$\mathbf{S}$

SC-PCF

SDS

SERS

SPR

$\mathbf{T}$

TEM

TPR
Thiolated Polyethylene Glycol

Polyethylenimine

Poly (ethylene oxide)

Pluronic Triblock Copolymer

Perfectly Matched Layer

Poly (propylene oxide)

Radio Frequency

Side Channel Photonic Crystal Fiber

Dodium Dodecyl Sulfate

Surface Enhanced Raman Spectroscopy

Surafce Plasmon Resonance

Transmission Electron Microscopy

Transverse LSPR 


\section{List of Figures}

FIGURE 2.1 TEM IMAGES OF DIFFERENT SIZES OF AUNPS. (A) $5.5 \pm 0.6$, (B) $8.0 \pm 0.8$, (C) $17 \pm$

$$
\text { 2.5, AND (D) } 37 \pm 5 \mathrm{NM}[17] \text {. }
$$

FIGURE 2.2 TEM IMAGES OF AUNRS WITH AN LSPR ABSORPTION PEAK AT (A) 700, (B) 760, (C) 790, (D) 880, (E) 1130, AND (F) 1250 NM. THE SCALE BAR IS 50 NM [18].

FIGURE 2.3 SPR SENSORS TYPICALLY UTILIZE ABSORPTION WAVELENGTH DEPENDENT UPON THE REFRACTIVE INDEX (RI) OF THE SAMPLE. WHEN THE SAMPLE RI CHANGES, THE MODE SHIFTS SPECTRALLY. THE RI SENSITIVITY IS DETERMINED BY MEASURING THE SPECTRAL SHIFT OF THE RESONANT MODE FOR KNOWN CHANGES IN SAMPLE RI [31].

FIGURE 2.4 UV-VIS SPECTRA OF DIFFERENT-SIZED AUNPS [17].

FIGURE 2.5 LSPR ABSORPTION SPECTRA OF AUNRS CAN BE TUNED BY USING SINGLE(LABELS 1) AND TWO-COMPONENT (LABELS 2) SURFACTANT MIXTURES DURING SYNTHESIS [18].

FIGURE 2.6 SANDWICHED SERS SUBSTRATE FOR ATP DETECTION [50].

FigURE 2.7 (A) SEM TAPERED OPTICAL FIBER. (B) TEM OF AUNPS. (C) SEM OF NANOPROBE MODIFIED WITH AUNPS. (D) DISTRIBUTION OF AUNPS ON THE NANOPROBE SURFACE [54].

FIGURE 2.8 (A) SEM OF THE CROSS SECTION OF HC-PCF AND (B) SCHEMATIC OF THE BINDING OF SERS NANOTAGS TO IMMOBILIZED BIOMARKERS INSIDE THE CORE OF HC-PCF FOR MULTIPLEX DETECTION [58]

FIGURE 2.9 IN VIVO CANCER TARGETING AND SERS DETECTION USING SCFV ANTIBODY CONJUGATED AUNPS THAT RECOGNIZE THE TUMOR BIOMARKER EGFR [77].

FIGURE 3.1 (A) NORMALIZED UV-VIS ABSORPTION SPECTRA OF AS-SYNTHESIZED AUNRS AND AUNRS AFTER DIFFERENT ROUNDS OF WASHING BY CENTRIFUGATION. (B) THE LONGITUDINAL SPR ABSORPTION WAVELENGTH FOR DIFFERENT WASHING ROUNDS. 38

FiguRE 3.2 TEM IMAGES OF (A) AS-SYNTHESIZED AUNRS, (B) AUNRS AFTER 4 TIME CENTRIFUGATION WASH, (C) PEGYLATED AUNRs AND (D) PEO-PPO-PEO ENCAPSULATED AUNRS. 40

FIGURE 3.3 RELATIVE CELL VIABILITY OF OSCC CELLS TREATED WITH AS-SYNTHESIZED AUNRS AND AUNRS AFTER DIFFERENT ROUNDS OF WASHING TREATMENTS FOR 24 HOURS POST-TREATMENT..

FIGURE 3.4 CELL VIABILITY OF AUNRS ENCAPSULATED WITH DIFFERENT CONCENTRATIONS OF PEG-SH OR PLURONIC TRIBLOCK COPOLYMER. THE CONCENTRATION OF PEG-SH AND PLURONIC ADOPTED FOR ENCAPSULATION SPANS FROM 10 NM TO 1 MM.

FIGURE 3.5 MEAN HYDRODYNAMIC DIAMETERS OF AS-SYNTHESIZED AUNRS (UNWASHED) AND AUNRS AFTER DIFFERENT ROUNDS OF CENTRIFUGATION WASH. HYDRODYNAMIC 
DIAMETER INFERS THE DIAMETER OF THE OUTERMOST ENCAPSULATION LAYER ON A PARTICLE.

FIGURE 3.6 MEAN HYDRODYNAMIC DIAMETER OF AUNRS ENCAPSULATED WITH DIFFERENT CONCENTRATIONS OF PEG-SH OR PLURONIC.

FIGURE 3.7 DARK-FIELD IMAGES OF OSCC CELLS WITH AUNRS ENCAPSULATED WITH (A) 10 NM, (B) $1 \mu \mathrm{M}$, (C) 1 MM OF PEG-SH AND (D) 10 NM, (E) $100 \mu \mathrm{M}$ (F) 1 MM OF PLURONIC F127.

FIGURE 4.1 (A) CTAB (BLACK SQUIGGLES) AS A STRUCTURE INDUCING AGENT DURING AUNRS FORMATION AS MORE GOLD IONS (YELLOW BLOCKS) ARE INTRODUCED. (B) THE PPO BLOCK OF PLURONIC BINDS WITH HYDROPHOBIC TAIL OF CTAB BY HYDROPHOBIC INTERACTIONS AND THUS ENCAPSULATES AUNRS.

Figure 4.2 (A) UV-VIS SPECTRA OF CTAB-COATED AUNRS (BARE NR), PLURONIC F-108 ENCAPSULATED AUNRS (F108-NR) AND PLURONIC F-127 ENCAPSULATED AUNRS (F127-NR). THE UV-VIS SPECTRA WERE TAKEN FROM AUNRS SUSPENDED IN HPLC WATER. TEM IMAGES OF (B) BARE AUNRS, (C) F108-AUNRS AND (D) F127-AUNRS. (CONCENTRATION OF PLURONIC: 20MG/ML).

FIGURE 4.3 BLUESHIFT VARIATION TREND WITH RESPECT TO THE LONGITUDINAL SPR PEAK OF THE ABSORPTION SPECTRA OF CTAB-COATED AUNRS (CTAB-GNR), F-108-COATED AUNRs (F108-GNR) AND F-127-COATED AUNRS (F127-GNR) STORED AT ROOM TEMPERATURE OVER 10 DAYS (THE CONCENTRATION OF PLURONIC IS 20MG/ML)...........55

FIGURE 4.4 (A) CELL VIABILITY OF BARE AUNRS (NR) (AFTER CENTRIFUGATION) AND AUNSS (NS), (B) CELL VIABILITY OF PLURONIC F-127 AND F-108 ENCAPSULATED AUNRS WITH DIFFERENT PLURONIC CONCENTRATIONS.

FIGURE 4.5 DIC MICROSCOPY IMAGES OF A) OSCC CELLS TREATED WITH CTAB-COATED AUNRS ARE SHOWN TO BE NON-VIABLE (FLATTENED STRUCTURE), B) OSCC CELLS TREATED WITH AUNRS ENCAPSULATED WITH F127 (30MG/ML) (ROUNDED STRUCTURE), SHOWS CTAB-COATED AUNRS ARE HIGHLY TOXIC TO THE CELLS WHILE PLURONIC-ENCAPSULATED AUNRS SHOW HIGHLY BIOCAPATIBILITY. (BAR = 100UM) ......57

FIGURE 4.6 DARK-FIELD IMAGES OF OSCC CELLS (A) WITHOUT AUNRS, (B) UPTAKING OF PLURONIC F-108 ENCAPSULATED AUNRS, AND (C) UPTAKING OF PLURONIC F-127 ENCAPSULATED AUNRS. HYPERSPECTRAL ABSORPTION SPECTRA OF (D) F-127 ENCAPSULATED AUNRS (POINTED OUT BY ARROWS IN FIGURE 4.6(B)) AND (E) F-108 ENCAPSULATED AUNRS (POINTED OUT BY ARROWS IN FIGURE 4.6(C)).

FIGURE 5.1 (A) THE NORMALIZED UV-VIS SPECTRA AND TEM IMAGES OF (B) AUNSS (GNS) WITH SPR AT 537 NM (C) AUNRs (GNR) WITH LONGITUDINAL SPR AT 626 NM AND (D) AUNRS (GNR) WITH LONGITUDINAL SPR AT 754 NM. (SCALE BAR=50 NM)

FIGURE 5.2 AUNPS (AUNRS AND AUNSS) FUNCTIONALIZATION PROCESS BY USING: (A) PEG-SH AND (B) HS-PEG-COOH, AND BIOCONJUGATION PROCESS BY USING (C) EDC, (D) NHS AND ANTI-EGFR ANTIBODY. 
FIGURE 5.4 TIME-DENPENDENT PROFILE OF THE EFFECTIVE DIAMETER OF THE PEGYLATED AUNSS (GNS) AND AUNRS (GNR) IN PBS BUFFER SOLUTION.

FIGURE 5.5 CELL VIABILITY OF BARE AUNSS, BARE AUNRS (AFTER WASHING), PEGYLATED AUNSS AND PEGYLATED AUNRS

FIGURE 5.6 DARK-FIELD IMAGES OF OSCC CELL LINE INCUBATED WITH (A) AUNSS WITH SPR PEAK AT 537 NM, (B) AUNRS WITH LONGITUDINAL SPR PEAK AT 626 NM, (C) AUNRS WITH LONGITUDINAL SPR PEAK AT 754 NM AND (D) A MIXTURE OF THREE TYPES OF AUNPS. THE HYPER-SPECTRA TAKEN FROM DARK-FIELD FROM (A), (B), (C) AND (D) WERE SHOWN IN (E), (F), (G) AND (H) RESPECTIVELY.

FIGURE 5.7 DARK-FIELD IMAGES OF (A) BARE OSCC CELL LINE AND (B) SKOV3 CELL LINE INCUBATED WITH ANTI-EGFR ANTIBODY CONJUGATED AUNRS.

FIGURE 6.1 (A) SCHEMATIC OF A SANDWITCHED SPR-ENHANCED BIO-SENSOR MODEL.(B) SANDWICHTED STRUCTURE FORMED BY AUNR, BIOMARKER AND GOLD SENSING FILM... 80

FIGURE 6.2 (A) SIMULATED ELECTRICAL FIELD DISTRIBUTION FOR AUNR OVER A 40 NM THICK GOLD FILM. AUNR HAS A WIDTH OF 20 NM AND LENGTH OF 70 NM, AND THE DISTANCE BETWEEN AUNR AND GOLD FILM IS 5 NM. (B) ENLARGEMENT OF THE ENHANCED ELECTRICAL FIELD AREA IN (A).....

FIGURE 6.3 THE SPR PEAK E-FIELD MODULUS FOR GOLD FILM THICKNESS VARIES FROM 10 NM TO 120 NM FOR DIFFERENT AUNRS SIZES.

FIGURE 6.4 THE VARIATION OF THE SPR E-FIELD MODULUS SPECTRA WITHOUT AUNR AND WITH AUNR BY VARING AUNR-TO-FILM DISTANCE

FIGURE 6.5 SPR PEAK WAVELENGTH FOR DIFFERENT AUNR WIDTH AND LENGTH ON GOLD FILM.85

FIGURE 6.6 SPR ABSORPTION SPECTRA FOR AUNRS WITH 20NM WIDTH AND ASPECTRATIO FROM 2 TO 4 ON 40NM GOLD FILM WITH 20NM DISTANCE.

Figure 7.1 ILLUSTRATION OF THE DETECTION METHOD. (A) CHEMICAL STRUCTURE OF SIALIC ACID ON CELL SURFACE. (B) THE CHEMICAL STRUCTURE OF DBA. (C) DBA-BONDED SIALIC ACID ON THE MEMBRANE OF A CELL AT THE C-8, 9 DIOL OF SIALIC ACID. (D) RAMAN SPECTRUM DETECTED FROM DBA TAGGED CELL IN SC-PCF, THE 2,000 CM-1 PEAK ARISES FROM ALKYNE GROUP FROM DBA. (E) SCHEMATIC OF SC-PCF AS A SERS PLATFORM FOR DETECTION OF SIALIC ACID ON A SINGLE CELL. (F) CHEMICAL STRUCTURE OF DBA, SIALIC ACID AND DBA BONDED SIALIC ACID.

FiguRE 7.2 SC-PCF BASED SERS DETECTION SETUP. (A) PHOTOGRAPH OF A SYRINGE CONNECTED SC-PCF FIBER. THE CONCENTRATION OF CELLS IN EACH SAMPLE WAS FIXED AT 20,000 CELLS/ML AND THEY WERE UNIFORMLY DISPERSED WHEN PUMPED INTO THE FIBER. THIS ENSURED THAT OUR DETECTION OF SIALIC ACID WAS RESTRICTED TO THE SINGLE CELL LEVEL. (B) THE FIBER WITH THE LIQUID SAMPLE PUMPED IN WAS MOUNTED ON THE MICROSCOPE STAGE USING AN SMA CONNECTOR AND LASER LIGHT WAS COUPLED INTO THE FREE-END OF THE FIBER THROUGH THE OBJECTIVE LENS

FIGURE 7.3 RAMAN ENHANCEMENT OF AUNP WITH DIFFERENT DIAMETERS. (A) RAMAN SPECTRA OF $100 \mu \mathrm{M}$ DBA WITH DIFFERENT AUNP DIAMETERS TESTED ON GLASS SLIDES XIX 
AND $100 \mu$ M DBA WITH 60 NM AUNP TESTED IN SC-PCF. (B) TEM IMAGE OF 60 NM AUNP. (C) CALCULATED |E|/|E0| OF 60 NM AUNP UNDER 633 NM LIGHT EXCITATION. (D) RAMAN INTENSITY OF $100 \mu$ M DBA WITH DIFFERENT AUNP DIAMETERS (BLACK BAR PLOT) AND THE CALCULATED RAMAN ENHANCEMENT FACTOR FOR AUNP OF DIFFERENT DIAMETERS (RED LINE)

FIGURE 7.4 COMPARISON BETWEEN RAMAN SIGNAL INTENSITY IN SC-PCF AND HC-PCF. MiCROSCOPE IMAGE OF A CROSS SECTION OF (A) SC-PCF AND (B) HC-PCF. (C) THE CALCULATED E-FIELD DISTRIBUTION OF SC-PCF WHEN THE SIDE CHANNEL IS FILLED WITH WATER (WAVELENGTH AT 633 NM). (D) ENLARGED IMAGE OF E-FIELD DISTRIBUTION IN FIBER CORE. (E) THE RAMAN SPECTRA OF $1 \mu \mathrm{M}$ NT IN 60 NM AUNP SOLUTION TESTED USING SC-PCF AND HC-PCF

FiguRE 7.5 (A) RAMAN SPECTRA OF $100 \mu$ M DBA WITH 60 NM AUNP (RED LINE) AND HELA CELL (BLUE LINE) IN SC-PCF. (B) RAMAN SPECTRA OF DIFFERENT CONCENTRATIONS OF DBA WITH 60 NM AUNP IN SC-PCF. 2,000 CM-1 PEAK INTENSITIES FOR DIFFERENT CONCENTRATIONS OF DBA WERE MEASURED FOR CALIBRATION.

FIGURE 7.6 DARK-FIELD IMAGE OF SINGLE HELA CELL SURROUNDED BY 60NM AUNP PUMPED OUT FROM SYRINGE-CONNECTED SC-PCF OF FOUR STUDIED CASES: (A) A HELA CELL SUCCESSIVELY TREATED WITH PREDNISOLONE AND DBA (HEREIN REFERS TO (+)SIALIC ACID)). (B) A HELA CELL SUCCESSIVELY TREATED WITH PBA AND DBA (HEREIN REFERS TO (-)SiAliC ACID)). (C) A NORMAL HELA CELL TREATED WITH DBA AS POSITIVE CONTROL (HEREIN REFERS TO SIALIC ACID) AND (D) A NORMAL HELA CELL WITHOUT DBA TAG AS NEGATIVE CONTROL (HEREIN REFERS TO CELL ONLY).

FIGURE 7.7 SERS DETECTION AND QUANTIFICATION OF SINGLE HELA CELL. (A) RAMAN SPECTRA OF (+)SIALIC ACID SAMPLE, (-)SIALIC ACID SAMPLE, POSITIVE CONTROL (SIALIC ACID), NEGATIVE CONTROL (CELL ONLY) AND RAMAN SIGNAL FROM THE FIBER WHEN NO CELL WAS LOADED. (B) 2000 CM-1 PEAK INTENSITY OF (+)SIALIC ACID AND SIALIC ACID SAMPLES. (C) CALCULATED NUMBER OF SIALIC ACID MOLECULES (RED SQUARE FOR SIALIC ACID AND (+)SIALIC ACID SAMPLES) FROM PLOTTED TREND LINE (BLACK LINE) OF THE CALIBRATION POINTS (BLACK DOTS) (THE X AXIS IS IN LOG SCALE).111

FIGURE 8.1 SCHEMATIC ILLUSTRATION OF TAGGED AUNPS BIDING ON BMFON AFTER MMP-2/MMP-7 ENZYME CLEAVING OF PEPTIDES ON BMFON AND AUNPS 119

FIGURE 8.2 BMFON CHARACTERIZATION. SEM IMAGING OF (A) CLEAN BMFON SUBSTRATE (B) PEPTIDE SHIELDED BIOTIN-AVIDIN CONJUGATED BMFON AND (C) AUNPS BOND BMFON AFTER ENZYME CLEAVING. (D)(E)(F) ENLARGEMENT OF IMAGES FROM THE AREA (AREA WITHIN WHITE SQUARES) IN (A)(B)(C), RESPECTIVELY.(G) SERS MAPPING OF PEPTIDE CONJUGATED BMFON. (H) SERS MAPPING OF PEPTIDE CONJUGATED BMFON AFTER ENZYME CLEAVAGE. (E) RAMAN SPECTRA OF MMP PEPTIDE BOUND BMFON SUBSTRATE BEFORE AND AFTER ENZYME CLEAVING.

FIGURE 8.3 DETECTION OF INDIVIDUAL MMP ENZYMES. CHEMICAL STRUCTURE AND RAMAN SPECTRA OF (A) ATP AND (B) NT. SERS SPECTRA OF (C) BIOTIN-PEG-SH BOUND BMFON AND (D) NEUTRAVIDIN-BIOTIN-PEG-SH BOUND BMFON. (E) SERS SPECTRA OF ATP TAGGED AUNPS BOUND MMP-7 CONJUGATED BMFON AFTER INCUBATED WITH 
DIFFERENT CONCENTRATIONS OF MMP-7 ENZYME. (F) SERS SPECTRA OF NT TAGGED AUNPS BOUND MMP-2 CONJUGATED BMFON AFTER INCUBATION WITH DIFFERENT CONCENTRATIONS OF MMP-2 ENZYME. (G) INTENSITY CURVE OF THE ATP RAMAN PEAK AT 1584 CM-1 (X-AXIS IS LOG10 SCALED). (H) INTENSITY CURVE OF THE NT RAMAN PEAK AT 1377 CM-1 (X-AXIS IS LOG10 SCALED)

FIGURE 8.4 MULTIPLEXED DETECTION OF MMP-2 AND MMP-7 ENZYMES. RAMAN SPECTRUM OF MMP-2 AND MMP-7 SHIELDED BMFON SUBSTRATE SAMPLES IN THE PRESENCE OF (A) CONTROL SOLUTION CONTAIN NO ENZYME (B) MMP-2 ENZYME (C) MMP-7 ENZYME (D) BOTH MMP-2 AND MMP-7 ENZYME. 


\section{List of Tables}

TABLE 4.1 TABLE OF TRANSVERSE LSPR(TPR) AND LONGITUDINAL LSPR(LPR) WAVELENGTH OF AUNRS SYNTHESIZED WITH VARIED AGNO3 AND SEED VOLUMES.

ENVIRONMENTAL CONDITION FOR EACH SYNTHESIS WAS KEPT CONSISTENT.

TABLE 8.1 TABLE OF PEAK ASSIGNMENT OF RAMAN SPECTRA 


\section{Chapter 1 Introduction}

\subsection{Background and Motivation}

AuNPs have drawn abundant attention over the past several years due to their unique properties. First of all, AuNPs are typically tens of nanometers in diameter, and thus much smaller than cells. Therefore, they can be conjugated with antibodies and bind to cell surfaces or be taken up by cells.

Second, AuNPs (especially AuNSs and AuNRs) can be easily synthesized based on proposed protocols. Generally, AuNSs are synthesized by reducing the $\mathrm{Au}^{3+}$ ions in gold chloride solution into neutral $\mathrm{Au}$ atoms through the addition of reducing agents. In contrast, AuNRs are grown from gold seeds and subsequently reduced from gold salt with a weak reducing agent (ascorbic acid) in a micellar medium. During this process, cetylmethylammonium bromide (CTAB) acts as a structure-directing agent that linearly "zips" up gold ions to form the rod shape. Synthesized AuNSs and AuNRs formed by these methods can be stable for months.

Furthermore, AuNPs have very strong LSPR, which gives them powerful scattering and absorption properties. What is more noteworthy is that their LSPR wavelength is very sensitive to the shape and size of the particles; thus, the LSPR wavelengths of AuNPs, especially AuNRs, have good tunability. All of these 
properties provide AuNPs with a great potential for biological applications, especially bio-sensing and bio-imaging.

AuNRs are synthesized with the help of CTAB as a structure-inducing agent. However, it has been suggested that CTAB forms a tightly bound cationic bilayer on AuNRs with the cationic trimethylammonium head group exposed to the aqueous media. CTAB exposure cytotoxicity to cells and this toxicity issue of AuNRs hinders their further applications in vitro and in vivo. There is a strong demand for the development of plasmon scattering probes for multiplex dark-field imaging of live cancer cells, but only a handful of manuscripts have reported the use of AuNPs as probes. Furthermore, it has been reported that AuNPs can be used to enhance the sensitivity of SPR bio-sensors due to their strong LSPR effects. However, the detailed effects of AuNRs on SPR bio-sensors have not been fully studied.

Raman spectroscopy is one of the few important analytical tools that not only provides qualitative and quantitative information of the "finger print" spectra of small molecules, but also overcome various limitations experienced with other techniques. For example, in infrared spectroscopy, as most molecules are soluble in water, getting their spectral information is challenging due to absorption from water, and for more sensitive method such as mass spectroscopy and nuclear magnetic resonance, the cost can be much higher and the usage are much more difficult. Unfortunately, Raman signals are commonly very weak due to the 
inherently small Raman scattering cross sections of many molecules of interest. AuNPs have been used to enhance weak Raman signals, and thus achieve surface enhanced Raman spectroscopy (SERS). SERS produces very large enhancements in the effective Raman cross section of analytes when they are located in strong electromagnetic fields ("hotspots") generated upon excitation of LSPR by gold nanoparticles/nanostructures. This SERS enhancement strongly depends on the size and shape of the nanoparticles and the cluster structures they form. AuNPs serving as SERS-active substrate usually exhibit an average enhancement factor of $10^{3}-10^{6}$, but the enhancement factor for some localized points can exceed $10^{8}[1]$ when the Raman molecules are located exactly in the "hotspots". However, for complex surfaces on which multiple collective LSPRs from non-uniform nanostructures (aperiodic nanostructure patterns caused by fabrication limit or randomly disperse of colloid solution) on fabricated SERS substrates and colloid-based solutions are present, it is not easy to achieve high and reproducible SERS enhancement. Furthermore, due to the complex nature of the properties of bio-samples, developing a SERS-based sensing platform that is able to specifically capture and detect bio-samples such as proteins and antibodies remains a challenge.

As a result, many fields can benefit from the further optimization of AuNP surface chemistry and optical properties for bio-sensing and bio-imaging applications. 


\subsection{Objectives}

The objectives of this study were:

(i) To optimize the synthesis of biocompatible plasmonic AuNPs formulations with different shapes, sizes, and surface coatings;

(ii) To develop AuNPs-based label free plasmon scattering probes for dark-field imaging of cancer cells;

(iii) To engineer and study AuNRs-based SPR nano-biosensors for SPR bio-sensing of proteins;

(iv) To develop ultrasensitive SERS-based biosensors using AuNPs combined with optical fibers or SERS substrate.

\subsection{Major Contributions of The Thesis}

The major contributions of this thesis are listed as follows.

1. A systematic review of the literature on the preparation of AuNPs for bio-imaging and bio-sensing applications was conducted, including a detailed discussion of the synthesis methods. The history and evolution of the approaches for preparing AuNRs, AuNSs, and other types of AuNPs were introduced. The optical properties of AuNPs and the latest progress in bio-applications based on AuNPs were also reviewed and discussed, including:

(i) localized surface plasmon resonance (LSPR) properties of AuNPs; (ii) 
SERS properties and AuNPs applications in substrate- and fiber-based SERS;

(iii) Biological applications of AuNPs.

2. The cytotoxicity and optical properties of AuNRs functionalized with thiolated polyethylene glycol (PEG-SH) or Pluronic triblock copolymer (PEO-PPO-PEO) molecules were investigated. The morphology of these functionalized AuNRs was then characterized by UV-vis absorption spectroscopy, transmission electron microscopy (TEM), and dynamic light scattering (DLS). It was found that the synthesized AuNRs surfaces were terminated with CTAB molecules. Such a formulation causes adverse toxicity to cells, whereas the complete removal of CTAB molecules from AuNRs surface renders the AuNRs structurally unstable and causes particles to aggregate. PEG-SH and PEO-PPO-PEO were individually investigated as capping agents to reduce the cytotoxicity of AuNRs formulations while maintaining the optical, colloidal, and structural properties of the AuNRs. The results showed that encapsulating AuNRs with the PEG-SH or PEO-PPO-PEO molecules guaranteed the stability and biocompatibility of the nano-formulation. The use of the functionalized AuNRs as scattering probes for dark-field imaging of cancer cells demonstrated their good biocompatibility. These discoveries offer a unique solution for the development of safe scattering color probes for clinical applications such as the long-term imaging of cells and tissues. 
3. The following detailed studies of the use of AuNPs as scattering probes for dark-field imaging of cancer cells were conducted: (i) Prepared AuNRs were encapsulated with Pluronic F-108 and F-127 to reduce their cytotoxicity and improve their colloidal and optical stability. The uptake of Pluronic-encapsulated AuNRs by oral squamous-cell carcinoma (OSCC) cells was investigated using dark-field imaging. The results suggested that a large quantity of Pluronic-functionalized AuNRs were internalized through non-specific uptake. A local absorption spectra analysis was performed on cancer cells and the measured spectra confirmed that the observed scattered color spots originated from the Pluronic-encapsulated AuNRs. (ii) The use of PEG-SH encapsulated AuNRs as plasmon scattering probes for the targeted dark-field multiplex imaging of live cancer cells was proposed. By carefully engineering the surfaces, aqueous dispersions of anti-epidermal growth factor receptor (EGFR) antibody-conjugated AuNSs and AuNRs were prepared. The receptor-mediated delivery of antibody-conjugated AuNPs into the EGFR receptor-positive OSCC cell line was demonstrated using the dark-field microscopy technique. The results suggested that the AuNSs and AuNRs formulations could provide up to seven types of plasmon scattering probes based on their tunability range with a $100 \mathrm{~nm}$ spectral separation of absorption bands. 
4. Finite element method (FEM) simulations were performed to optimize the configuration of the AuNRs-enhanced SPR sensor and investigate its potential for multiplex antigen detection. The near-field coupling between the sensing film and AuNRs was analyzed. By systematically studying the effect of gold film thickness, AuNR-to-film distance, and AuNR dimensions on SPR, it was found that a change in length of AuNRs less than $40 \mathrm{~nm}$ wide caused a significant SPR wavelength shift of the sensor, while the sensor was insensitive to the AuNR-to-film distance. AuNRs with width of $20 \mathrm{~nm}$ and aspect ratios ranging from 2 to 4 were adopted to demonstrate the concept of conjugating gold film and AuNRs with anti-Immunoglobulin G (anti-IgG) antibodies for the multiplex detection of various IgG proteins with more than $100 \mathrm{~nm}$ separation of their SPR wavelengths

5. An ultrasensitive SERS-based sensing platform was developed to detect the mean sialic acid level on the surface of single cell with sensitivity as low as 2 femtomoles. This platform adopted the use of an interference-free Raman tag, 4-(dihydroxyborophenyl) acetylene (DBA), which selectively binds to the sialic acid on the cell membrane. By loading the side channel of a photonic crystal fiber with a mixture of $60 \mathrm{~nm}$ AuNPs and DBA-tagged HeLa cells, and subsequently propagating laser light through the central solid core, a strong SERS signal was obtained. Based on this technique, accurate detection and 
quantification of the concentration of sialic acid on single cell was achieved, surpassing previously reported methods that required over $10^{5}$ cells.

6. A SERS-based platform for the sensitive multiplexed detection of matrix metalloproteinases (MMP) (MMP-2 and MMP-7) with a low detection limit and high specificity was developed. The detection employed enzymatic reactions by which a peptide can be cleaved only by its corresponding enzyme. The platform comprised two components, a specialized SERS-based bimetallic film over nanosphere (BMFON) substrate and AuNPs. The detection was based on the cleave-and-bind mechanism, which was validated by the individual detection and quantification of MMP-2 and MMP-7. The platform was demonstrated to sensitively detect concentrations of specific enzymes ranging from $1 \mathrm{ng} / \mathrm{mL}$ to $40 \mu \mathrm{g} / \mathrm{mL}$. The multiplexed detection of MMP-2 and MMP-7 was also demonstrated. The multiplexity of this platform provides a robust way to analyze diseases associated with MMP-2 and MMP-7 enzymes.

\subsection{Organization of Report}

This report is organized as follow.

Chapter 1 presents a brief introduction to the background and motivation for AuNP research. The major contributions of the thesis, objectives of the project, and structure of the report are then described. 
Chapter 2 provides a literature review on the history and evolution of AuNPs synthesis, introduces basic knowledge of the LSPR and SERS of AuNPs, and investigates their biological applications.

Chapter 3 investigates the cytotoxicity, colloidal stability, and optical properties of AuNRs before and after functionalization with PEG-SH and Pluronic triblock copolymer (PEO-PPO-PEO) molecules. The morphology of functionalized AuNRs was characterized using UV-vis absorption spectroscopy, transmission electron microscopy, and DLS.

Chapter 4 describes how the AuNRs were synthesized and why CTAB-based AuNRs are toxic to cells. The mechanism of using Pluronic triblock copolymers to encapsulate AuNRs to reduce cytotoxicity, thereby improving colloidal and optical stability, is also explained. The experimental procedures are then described. Biocompatible optical probes based on Pluronic-encapsulated AuNRs under differential interference contrast and dark-field imaging is studied.

Chapter 5 introduces the use of PEG-SH-encapsulated AuNPs as plasmon scattering probes for dark-field multiplex imaging of targeted live cancer cells. The experimental implementations for antibody conjugation and receptor-mediated delivery into the EGFR receptor-positive OSCC cell line of AuNSs and AuNRs are demonstrated. Dark-field imaging of cancer cells using multiplexing probes is then investigated. 
Chapter 6 describes the use of FEM simulations to optimize the configuration of the AuNRs-enhanced SPR sensor and investigates its application for multiplex antigens detection. The effects of gold film thickness, AuNRs-to-film distance, and AuNRs dimensions on SPR are studied. The concept of using different aspect ratios of antibody-conjugated AuNRs for multiplex IgG protein sensing is described.

Chapter 7 proposes an ultrasensitive SERS-based sensing platform for detecting the mean sialic acid level on the surface of single cell. The combination of a uniquely designed side channel photonic crystal fiber and $60 \mathrm{~nm}$ AuNPs resulted in strong SERS enhancement, which made single cell detection possible.

Chapter 8 introduces a SERS-based platform for the sensitive multiplexed detection of MMP-2 and MMP-7 with a low detection limit and high specificity. The multiplex nature of this platform provides a robust way to analyze diseases associated with MMP-2 and MMP-7 enzymes.

Chapter 9 provides the conclusions and suggestions for future research. 


\section{Chapter 2 Literature Review}

\subsection{Introduction}

Nanoparticles are particles between 1 and $100 \mathrm{~nm}$ in size. Plasmonic nanoparticles, especially AuNPs, have received great attention over the past few decades due to their unique tunable optical properties. AuNPs include AuNSs, AuNRs, and other gold nano-structures, which are typically tens of nanometers in size and comparable to some biomolecules such as proteins and antibodies. However, they are much smaller than human cells. These nanoparticles can be conjugated with biomolecules for targeted imaging and sensing, and thus are widely used in applications such as photo-thermal therapy [2-4], photodynamic therapy [5], bio-imaging [6], biosensors [7], fluorescence enhancement [8], SERS [9], and drug and gene delivery [10].

AuNP synthesis and functionalization techniques have been evolving. However, certain challenges must be overcome before they can be successfully used in clinical research. For example, the challenge of synthesizing AuNPs with high reproducibility and stability hinders their application in AuNPs-based bio-sensing assays and affects their long-term shelf stability. The cytotoxicity and specificity of AuNPs require improved surface functionalization techniques so that AuNPs can be used for 
bio-applications such as bio-imaging, photo-thermal therapy, and in vivo and in vitro drug and gene delivery.

In this chapter, we first review and discuss AuNP synthesis techniques. More specifically, we describe the synthesis of AuNSs and AuNRs. In addition, SPR and SERS of AuNPs and their applications are described. AuNSs and AuNRs are particularly attractive for SPR and SERS due to their flexible plamonic properties, which strongly depend on particle shape and size and the dielectric constant of the surrounding media. The final section briefly describes the biological applications of AuNPs.

\subsection{AuNPs Synthesis}

The first studied AuNPs systems were AuNSs and they were previous named gold colloid during olden days. Since ancient times, AuNPs have commonly been used for decorative purposes and were often embedded in glassware, causing the glass to have an intense ruby-like color. However, at that time, it was unclear how gold colloids contributed to this coloring effect. The understanding of the optical properties of gold colloids did not begin until the late 1850s [11], when Michael Faraday pointed out that the ruby-like coloring effect was due to the minute size of the gold particles. In 1898, Richard Adolf Zsigmondy synthesized the first gold colloid-based AuNSs in a dilute solution. Then, in 1908, Gustav Mie explained the visible light absorption by gold colloids using Maxwell's electromagnetic equations [12]. Since then, the 
AuNP-research community has grown tremendously, and the optical properties of AuNP can be easily tuned for specific applications ranging from solar energy to medicine.

Generally, AuNSs are synthesized by reducing $\mathrm{Au}^{3+}$ ions in gold chloride solution into neutral $\mathrm{Au}$ atoms through the addition of reducing agents. During the reaction, large quantities of $\mathrm{Au}$ atoms in the solution are reduced from $\mathrm{Au}^{3+}$. When the atoms become over-saturated in the solution, they begin to aggregate and form sub-nanometer gold "seeds". As the seeds start to form in the solution, the rest of the $\mathrm{Au}$ atoms continue to grow on the seeds. Thus, the seeds become larger over time and eventually form AuNSs. Vigorous stirring is required to form even size distributions. Many methods have been proposed to synthesize AuNSs. Turkevitch et al. demonstrated the preparation of AuNSs using citrate to reduce $\mathrm{Au}^{3+}$ ions to $\mathrm{Au}$ atoms in 1951 [13], and this method has since been widely adopted to make AuNPs. In 1973, Frens reported the synthesis of AuNSs of certain sizes (16-147 nm) by varying the ratio between the reducing and stabilizing agents to control formation (Figure 2.1) [14]. The creation of AuNSs with high colloidal stability was demonstrated by Giersig and Mulvaney in 1993. With this method, AuNSs are stabilized by thiols of different chain lengths through the $\mathrm{Au}-\mathrm{S}$ bond between the soft acid Au and the soft thiolate base [15]. According to hard and soft acids and bases (HSAB) theory, soft acid and soft base applie to species which are big, have low charge states and are strongly polarizable, and they are able 
to form strong covalent bond (e.g. Au-S bond). Soon after, the Shiffrin-Brust biphasic synthesis method was published, and this approach is used to prepare organic-phase dispersible AuNPs [16]. This process involves gold chloride $\left(\mathrm{AuCl}_{3}\right)$, thiol, tetraoctylammonium bromide $\left(\left[\mathrm{CH}_{3}\left(\mathrm{CH}_{2}\right)_{7}\right]_{4} \mathrm{~N}(\mathrm{Br})\right)$, and $\mathrm{NaBH}_{4}$ in water-toluene yielding thiolate-AuNSs. These AuNSs can be dispersed in common organic solvents without any aggregation or deformation. More importantly, they can be transferred to the aqueous phase and functionalized with different thiol functional groups for bio-conjugation purposes.
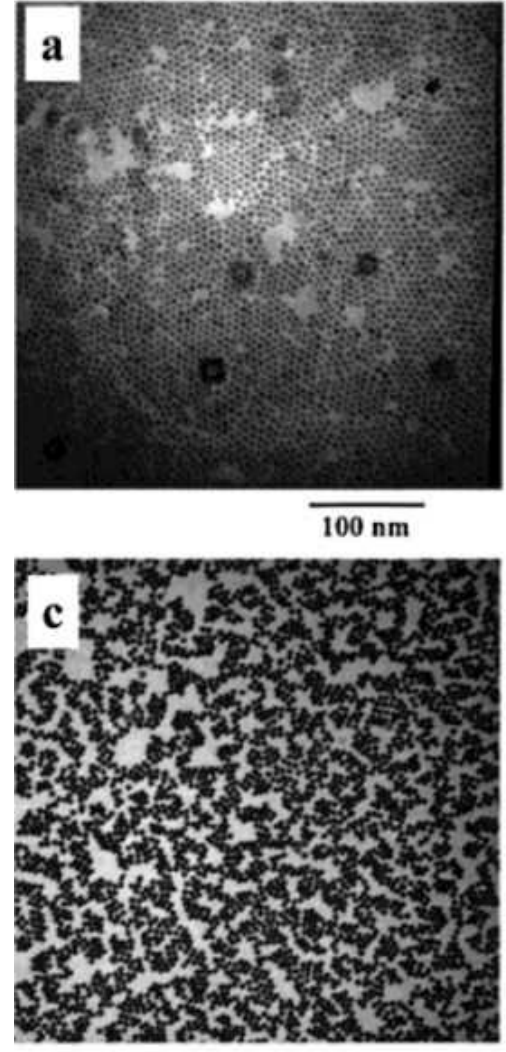

$\overline{100} \mathrm{~nm}$

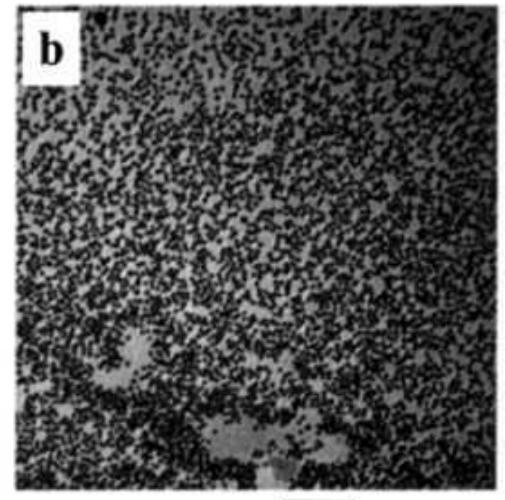

$\overline{100 \mathrm{~nm}}$

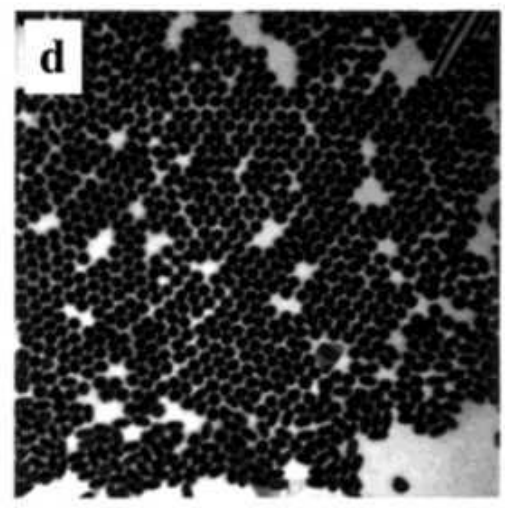

$1 \overline{00} \mathrm{~nm}$

Figure 2.1 TEM images of different sizes of AuNPs. (a) $5.5 \pm 0.6$, (b) $8.0 \pm$ 0.8, (c) $17 \pm 2.5$, and (d) $37 \pm 5 \mathrm{~nm}[17]$. 


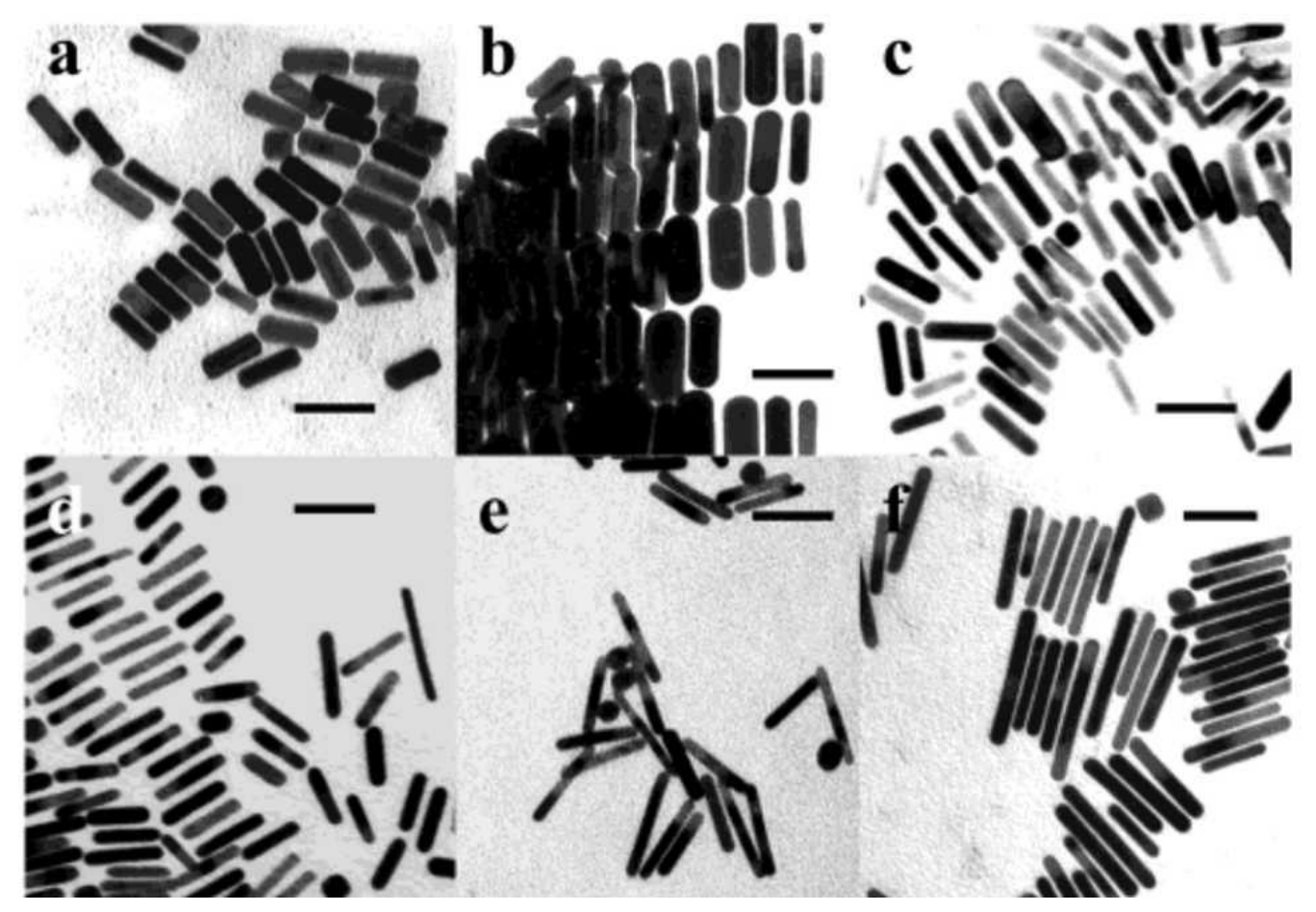

Figure 2.2 TEM images of AuNRs with an LSPR absorption peak at (a) 700, (b) 760, (c) 790, (d) 880, (e) 1130 , and (f) $1250 \mathrm{~nm}$. The scale bar is 50 $\mathrm{nm}[18]$.

Besides AuNSs, AuNPs with other sizes and shapes have been demonstrated by various research groups around the globe. For example, AuNRs have been intensively studied during the past few years due to their fascinating optical properties compared with AuNSs. Murphy's and El-Sayed's groups are among the pioneers in demonstrating the controlled synthesis of AuNRs with different aspect ratios (length of long axis over width of the rod) using the micelle templated seed mediated growth method [18-20]. With this method, AuNRs can be grown in the presence of gold seeds by using the reduction of gold salt with a weak reducing agent (ascorbic acid) in a micellar medium. During the growth of AuNRs, the head group of the cationic surfactant CTAB 
preferentially binds to certain specific crystallographic faces of gold particles, thus forming a bilayer on the particle surface. Subsequently, the CTAB bilayer acts as a structure-directing agent that linearly "zips" gold ions to aid the formation of rod-shaped AuNPs. This results in AuNRs to have cationic charge on their surface. In general, the aspect ratio of AuNRs can be tuned from 2 to 8 by carefully modifying the amount of silver nitrate added to the growth micellar solution (Figure 2.2). AuNRs with high aspect ratios (e.g., 18-25) can be synthesized by carefully controlling the $\mathrm{pH}$ of the reaction solution [21]. Other gold nanostructures such as gold nanostars [22], gold nanoshells [23], and gold nanocubes [24] have also been studied over the last few years. However, AuNSs and AuNRs remain the primary research interest of the AuNP-research community because they can be easily prepared with standard reported protocols and functionalized with biomolecules for nano-medical applications.

\subsection{LSPR of AuNPs}

In general, SPR refers to the collective oscillation of electrons in a solid or liquid stimulated by incident light. The resonance occurs when the frequency of light photons matches the natural frequency of surface electrons oscillating against the restoring force of positive nuclei [12]. When SPR is excited, light with certain wavelength will be absorbed by the metal surface, and the absorption wavelength is highly sensitive to the refractive index of the metal 
and the contacting medium. This phenomenon has been utilized to develop SPR sensors, which detect the sample by measure the absorption wavelength dependent upon the refractive index (RI) of the sample (as shown in Figure 2.3). When light interacts with particles much smaller than the incident wavelength, it leads to a plasmon that oscillates locally around the particle with a frequency known as localized SPR (LSPR). LSPR typically occurs when light is incident on noble metal nanoparticles, such as gold and silver nanoparticles, and the resonance results in the enhanced absorption and scattering of the electromagnetic field at the nanoparticle surface, which can be characterized by UV-vis spectroscopy [25]. Because this electromagnetic field enhancement mechanism requires coupling of the incident radiation to the metal surface, the LSPR wavelength is highly sensitive to the size, shape, material, and local dielectric properties of nanoparticles [26-29]. For example, for AuNSs with a diameter of $5 \mathrm{~nm}$ and an SPR wavelength of $\sim 520 \mathrm{~nm}$, the particular LSPR wavelength can be tuned to $530 \mathrm{~nm}$ when the diameter of the AuNSs is increased to $40 \mathrm{~nm}$, as shown in Figure 2.4 [17]. More interestingly, the plasmon resonance wavelength is different when nanoparticles change from spherical to non-spherical shape. For example, two plasmon resonance wavelengths can be observed for AuNRs, one corresponding to wave oscillations along the length of the AuNRs (longitudinal LSPR) and the other along the width of the AuNRs (transverse LSPR). The wavelengths of these two resonances are dependent on the width and length of the AuNRs. Thus, 
the resonance peak wavelength of AuNRs can be tailored from 550 to 1400 $\mathrm{nm}$ by using one type of surfactant or two type of surfactants for applications ranging from photo-thermal therapy to dark-field imaging, as shown in Figure 2.5 [30]. For example, chromophores are light-absorbing substances within tissue. Most organic molecules display strong absorption in the UV region, thus penetration in this region is very weak. In the red and near infrared (600 to $1200 \mathrm{~nm}$ ) regions, light is weakly absorbed and penetrates deeply into the tissue. Therefore, the LSPR wavelength of AuNRs can be tuned within this range for photo-thermal therapy.

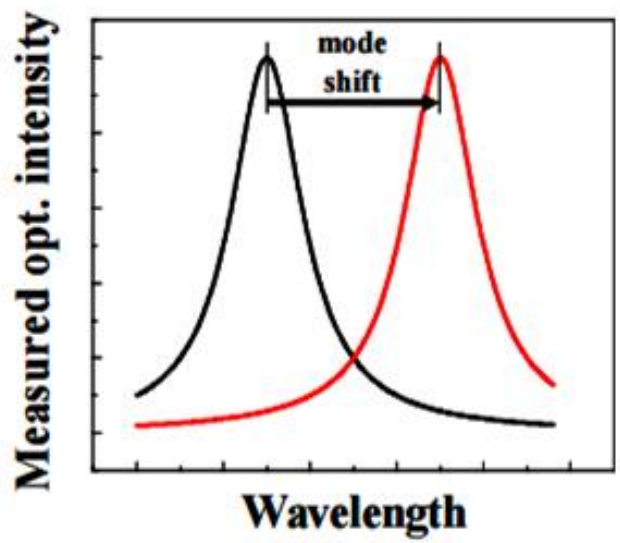

(A)

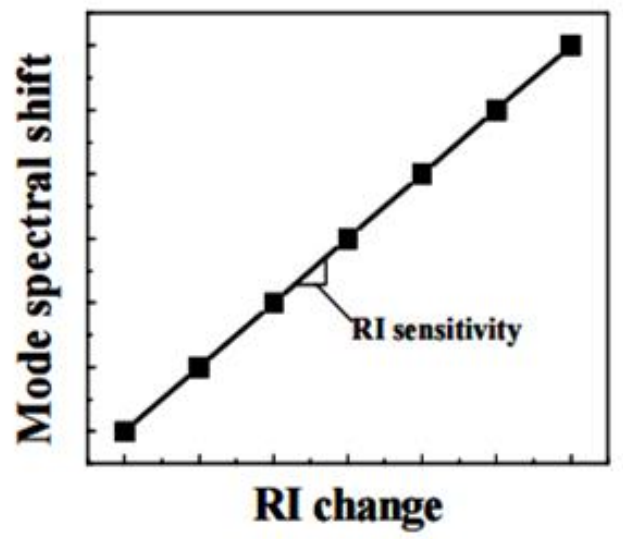

(B)

Figure 2.3 SPR sensors typically utilize absorption wavelength dependent upon the refractive index (RI) of the sample. When the sample RI changes, the mode shifts spectrally. The RI sensitivity is determined by measuring the spectral shift of the resonant mode for known changes in sample RI [31]. 


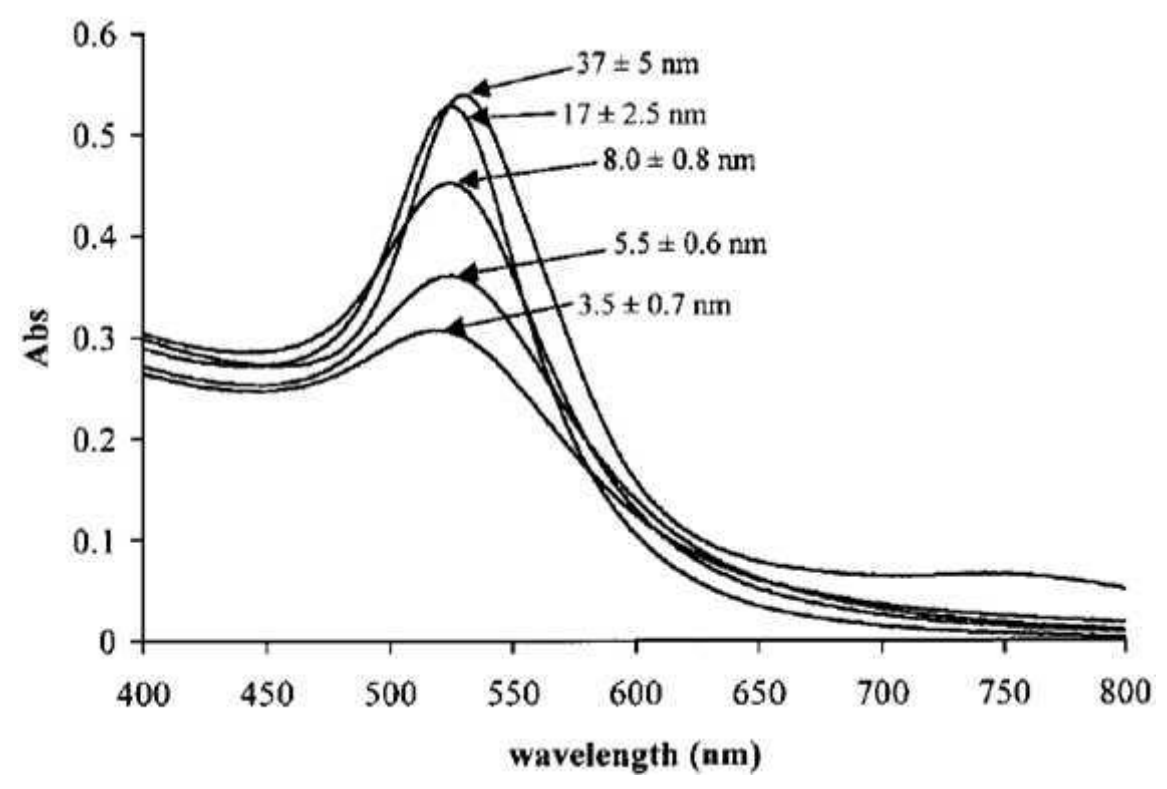

Figure 2.4 UV-vis spectra of different-sized AuNPs [17].

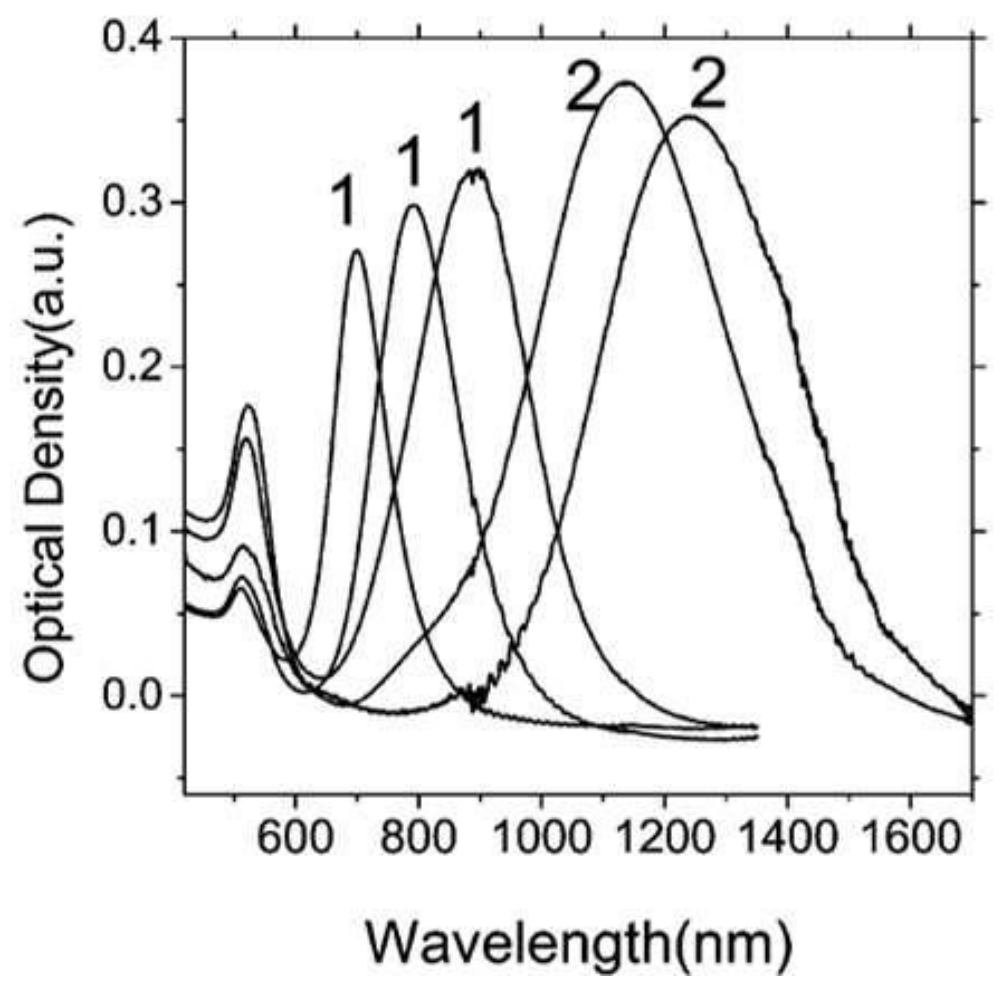

Figure 2.5 LSPR absorption spectra of AuNRs can be tuned by using single- (labels 1) and two-component (labels 2) surfactant mixtures during synthesis [18]. 


\subsection{SERS of AuNPs}

When incident light (electromagnetic waves) encounters molecules, the interaction between electromagnetic waves and molecules causes the electrons to orbit within the constituent molecules, oscillating periodically at the same frequency as the electric field of the incident wave. This oscillation of the electron cloud results in a periodic separation of charge within the molecules, which is called an induced dipole moment. The oscillating induced dipole moment is manifested as a source of electromagnetic radiation, thereby resulting in scattered light [32]. During scattering, most of light is scattered at the same frequency as the incident light, and this is referred to as Rayleigh scattering. Only a small fraction of light is scattered at different frequencies, this inelastic scattering is called Raman scattering. As the spectrum of the Raman-scattered light depends on the state of molecular constituents, Raman spectroscopy is widely used for the identification and analysis of specific biomolecules [33-35]. However, as the inelastic scattering of light from the molecule vibration is weak, the Raman signal is normally very weak and thus hampers its use for detecting low concentrations of biomolecules, for which a highly sensitive signal is required to examine the structure of the particular molecule. In 1974, Fleischmann, Hendra, and McQuillan discovered surprisingly strong and potential-dependent Raman signals from pyridine adsorbed on a silver electrode that had been electrochemically roughened in a potassium chloride aqueous electrolyte [36]. Jeanmaire and Van Duyne [37] 
and Albrecht and Creighton [38] later explained that the degree of the Raman signal enhancement was based on electromagnetic and chemical effects, respectively. Long-range electromagnetic enhancement is due to the plasmon resonance of incident light and the collective electron oscillations of the nanostructures, resulting in the strong enhancement of local electric fields at the particle surface. In contrast, short-range chemical enhancement is caused by the change in molecular polarization due to the charge-transfer interaction of the molecules with the metal surface. In 1978, Frederick W. King's group reported that the main cause of the Raman intensity enhancement is the electromagnetic effect [39], which contributes $10^{2}-10^{6}$ of the enhancement factor, while the chemical vibration only contributes $10^{1}-10^{2}$. This effect results in a total Raman signal enhancement proportional to the fourth power of the local electric field. Since then, SERS has received great attention because it can largely improve the detection limit at single-molecule level. AuNPs have been targeted for the development of this technique because the aggregates of nanoparticles are able to give rise to greater SERS enhancement. Several theories have suggested that the enhancement of the SERS signal is caused by the "hot spot" at the junction of two or more particles [40]. It has been observed that AuNRs provide higher SERS signals than those of AuNSs due to the higher electric field generated at the end of the rod [41]. Hence, AuNRs have been widely adopted for SERS detection. The shape of AuNRs can be adjusted with suitable aspect ratios so that their LSPR wavelength can 
correspond to commercial laser wavelengths in Raman systems (normally 633 $\mathrm{nm}$ and $785 \mathrm{~nm}$ ) to maximize the Raman enhancement [42]. Furthermore, AuNPs can be easily conjugated with Raman tags and biomolecules for SERS bio-applications such as targeting and imaging of cells [43, 44].

\subsubsection{Substrate-Based SERS}

Conventionally, SERS detection has been performed using a gold or silver nanostructured substrate, which allows the Raman signal to be enhanced by several orders of magnitude due to the strong LSPR from nanostructured surfaces [45]. Here, plasmonic nanoparticles were used to fabricate the substrate. A simple way to do this is to spray Au or Ag colloids on a surface, thus generating an extremely high SERS signal at some local "hot spot" [40]. However, it is difficult to achieve a reliable, stable, and uniform SERS signal spanning a wide dynamic range using this method. Van Duyne et al. proposed using nanosphere lithography [46], and Liu et al. used soft lithography [47] to fabricate nanoparticle-array substrate with high SERS activity and improved uniformity. García-Vidal's group theoretically investigated the relationship substrate nanostructure and SERS enhancement. They proposed that very localized plasmon modes, created by strong electromagnetic coupling between touching metallic objects, dominate the surface enhanced Raman scattering response [48]. Gaps between adjacent particles on substrate generate broad LSPR peaks, and thus the resonance covers both the excitation wavelength and the Raman peak, inducing strong SERS enhancement $\left(10^{5}-10^{6}\right)$. Their 
calculation of the enhancement factor of nanorods-array substrates indicated that a significant near-field interaction occurs between adjacent nanorods when the gap between the nanorods reaches half the value of their diameter. To obtain even greater SERS enhancement, Catherine J. Murphy proposed using a sandwiched architecture substrate [49], which incorporates AuNPs and substrate, between which the analytes are sandwiched. A $10^{9}$ enhancement factor was observed for the sandwich assays when LSPR-Surface plasmon polariton coupling occurred. A similar design was proposed by X. Hu et al. [50], who presented a universal metal-molecule-metal sandwich architecture created by the self-assembly of $\mathrm{Ag}$ and $\mathrm{Au}$ nanoparticles of various shapes interconnected with 4-aminothiophenol (ATP) molecules, as shown in Figure 2.6. For substrate-based SERS, as the enhancement is only effective when Raman tags are located very close to the substrate surface, the effective enhancement distance of the substrate is usually less than $100 \mathrm{~nm}$ [51-53], resulting in a short detection distance.

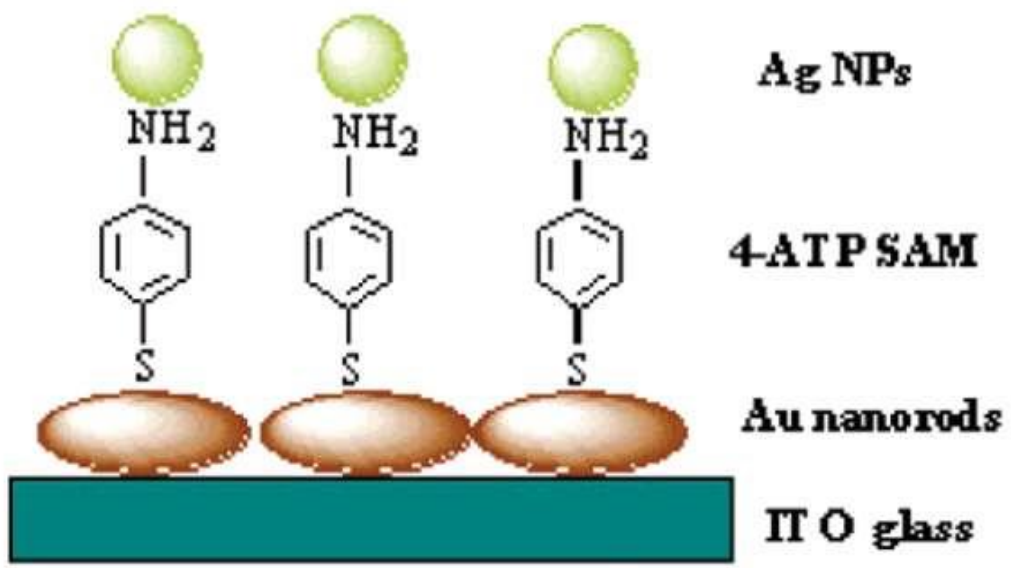

Figure 2.6 Sandwiched SERS substrate for ATP detection [50]. 


\subsubsection{Fiber-Based SERS}
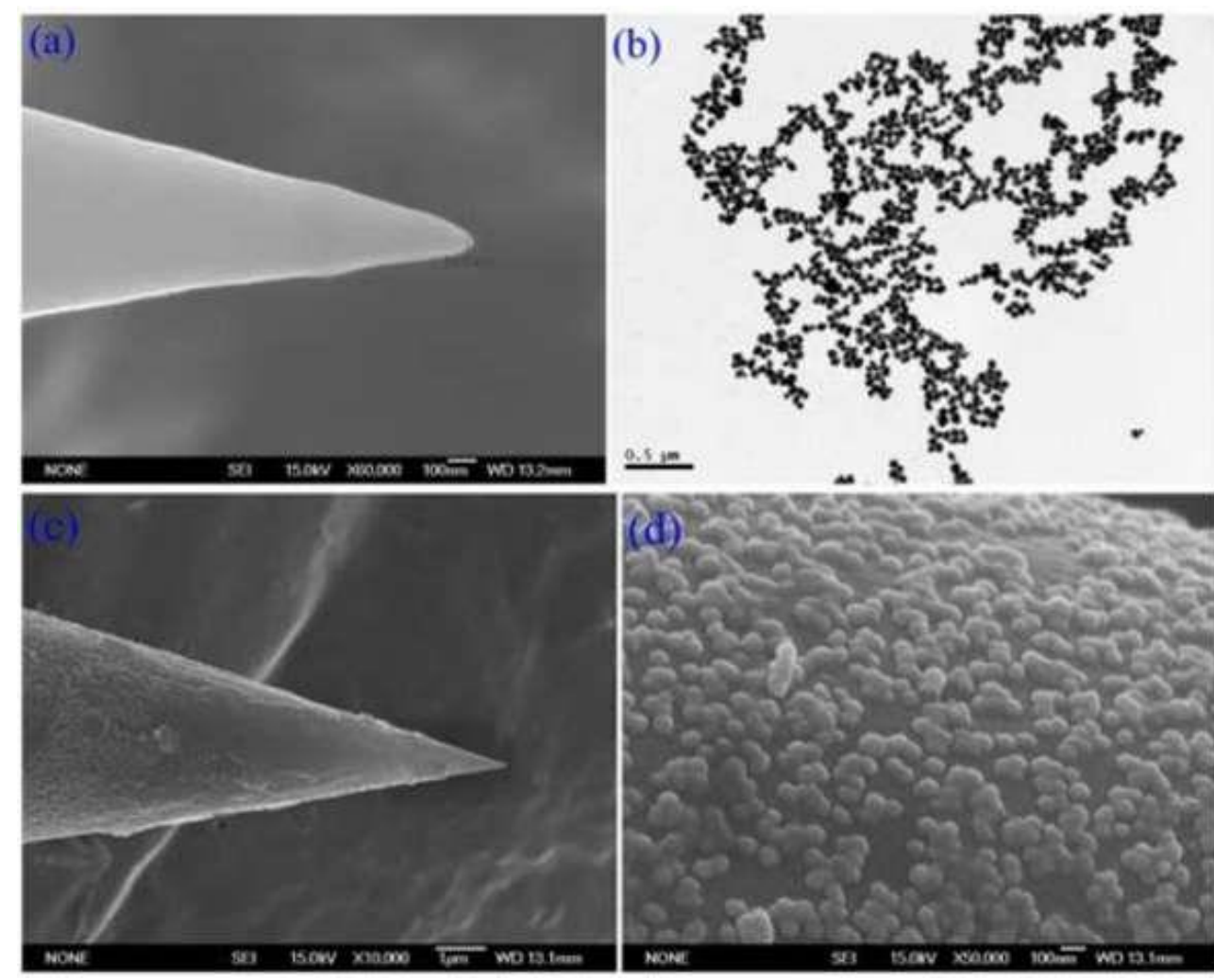

Figure 2.7 (a) SEM tapered optical fiber. (b) TEM of AuNPs. (c) SEM of nanoprobe modified with AuNPs. (d) Distribution of AuNPs on the nanoprobe surface [54].

To increase the detection distance and ease of use, molecular detection based on optical-fiber SERS has attracted extensive attention in recent years. The original SERS fiber probe was demonstrated in 1991 [55]. In the following years, studies involving different kinds of fiber tips were proposed. Tuan Vo-Dinh et al. developed a single-fiber SERS sensor by incorporating nanoparticles and silver coatings onto the fiber tip, thus allowing the fiber to dip into the sample solution for detection [56]. Andrea Lucotti et al. proposed an improved design, in which a tapered fiber tip was fabricated by means of a 
double static etching process. The SERS active fiber tip was prepared by the standard process of immobilizing nanoparticles, whereby the optimized geometry results in the best detection limits [57]. Recently, a similar SERS nanoprobe design was presented based on an AuNPs modified tapered optical fiber, which demonstrated its ability to perform remote detection [54]. The fiber tip was tapered to $40.7 \mathrm{~nm}$ by heated pulling and chemical etching methods. The AuNPs were deposited on the tip of the fiber with electrostatic self-assembly technology (Figure 2.7). This fiber probe can detect rhodamine $6 \mathrm{G}$ solution with a detection limit of $10^{-8} \mathrm{~mol} / \mathrm{L}$.
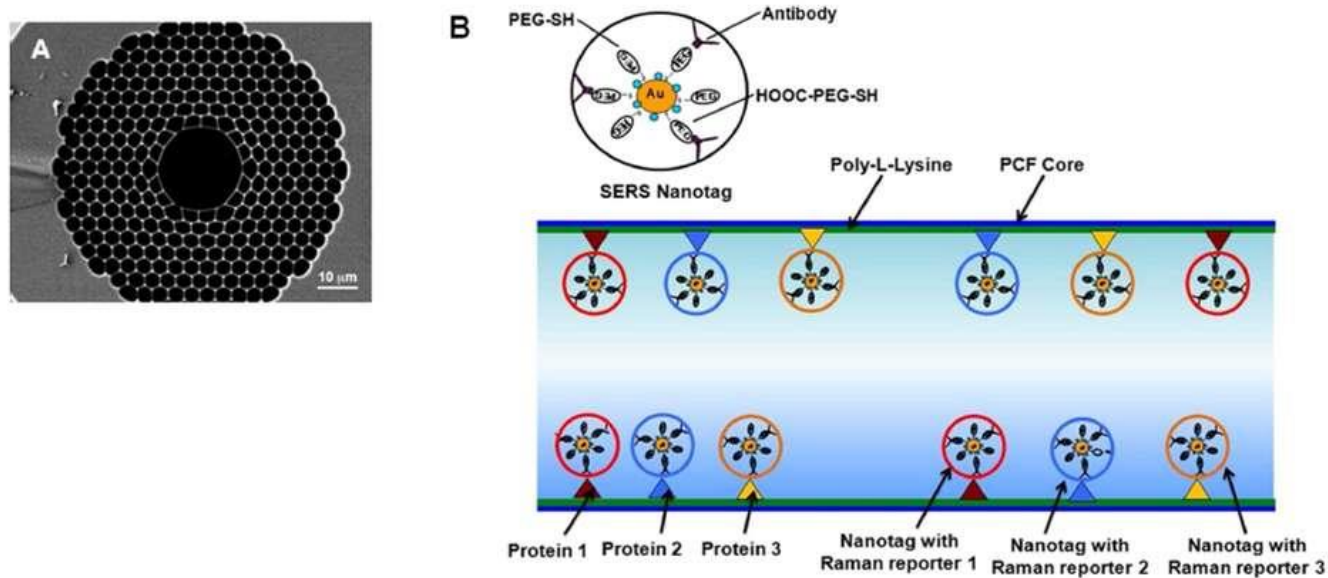

Figure 2.8 (a) SEM of the cross section of HC-PCF and (b) schematic of the binding of SERS nanotags to immobilized biomarkers inside the core of HC-PCF for multiplex detection [58].

Although these solid core fiber-based SERS probes were easy to develop, the small number of SERS substrate particles in the active region and weak light interaction with the analytes limit the sensitivity of these sensors. To involve more particles in the SERS activity and increase light interaction with the 
analytes, hollow core photonic crystal fiber (HC-PCF) has been adopted for SERS detection. He Yan et al. developed a fiber probe consisting of an HC-PCF with Au nanoparticles coated on the inner surface of the air holes serving as the SERS substrate [59]. The confinement of light inside the HC-PCF and the coating of nanoparticles/analytes inside the air holes of the HC-PCF offer a large area for the light to interact with the SERS particles throughout the length of the fiber, and therefore provide improved detection sensitivity. Similar designs were further investigated in the following years [60-62], one of which is shown in Figure 2.8. Compared to solid core fiber-based SERS probes, HC-PCF provides high sensitivity and lower fiber SERS background. However, the light is conducted in the HC-PCF by the band gap effect, which is highly sensitive to refractive index changes [63]. When the hollow core and air holes in the cladding are filled with liquid, the band gap shifts due to a refractive-index change [64]. As a result, guided light may not be well confined, leading to increased loss and decreased sensitivity. Therefore, this refractive index/guided wavelength sensitive nature of HC-PCF limits their application to liquid.

\subsection{Biological Applications of AuNPs}

AuNPs are able to scatter light with different colors due to SPR absorption, and the color can be tuned by changing the SPR wavelength through modifying the particle shape and size. These AuNPs can be easily detected by 
commercial microscopes under dark-field conditions [65]. In general, the SPR wavelength peak of AuNSs can be tuned from $524 \mathrm{~nm}$ to $572 \mathrm{~nm}$, thus enabling them to be observed under dark-field imaging. AuNRs have greater flexibility than AuNSs in terms of optical properties because their longitudinal SPR peaks red-shift when their aspect ratios increase. This unique feature of AuNRs, together with their excellent photostability [66], is well suited for bio-imaging and bio-sensing applications. For example, Sokolov's group demonstrated the use of anti-EGFR antibody conjugated AuNPs formulations for cancer cell imaging $[67,68]$ using standard light illumination conditions. AuNRs have also been adopted for fluorescence imaging, and the signal enhancement is due to the excitation of the longitudinal SPR that enhances the radiative rate of the inter-band electronic transitions. The quantum efficiency of AuNRs increases with rod length, and strong luminescence can be easily observed by the naked eye for AuNRs with a length of $200 \mathrm{~nm}[8,69,70]$. Wang's group demonstrated the use of single AuNRs for in vivo imaging by relying on their strongly induced luminescence from two-photon excitation using a femtosecond NIR laser [71]. In addition to imaging applications, AuNPs can be used as nano-heaters because they can strongly absorb light and convert the absorbed light to heat on a picosecond time domain through rapid electron-phonon and phonon-phonon processes [72]. Therefore, this light-heat conversion can be used to heat the local environment with light radiation of the same frequency as the nanoparticle SPR absorption 
wavelength, making them suitable for photo-thermal therapy. In 2006, El-Sayed's group demonstrated selective photo-thermal therapy of cancer cells based on anti-EGFR antibody conjugated AuNPs [73].

As AuNPs are able to greatly enhance the Raman signal, they are widely used in sensitive SERS bio-applications in vitro and in vivo. In vitro applications usually involve biomarker detection of DNA/RNA, proteins, and antibodies. They can also be applied for the molecular profiling of cells. Y.C. Cao's group proposed the multiplexed detection of oligonucleotide targets using AuNPs probes labeled with oligonucleotides and Raman active dyes. Raman fingerprints can be identified after SERS enhancement through AuNPs and silver substrate with a detection limit of 20 femto molar [74]. Huang et al. demonstrated antibody-conjugated AuNRs as potential cancer diagnostic markers using AuNRs surfactants as reporter molecules for the SERS signal [75]. Ultrasensitive SERS measurements in single living cells have also been demonstrated through exploiting $60 \mathrm{~nm}$ AuNPs that are deposited inside cells [76]. These SERS active nanoparticles result in strongly enhanced Raman signals of the native chemical constituents of the cells, thus allowing SERS measurements of a single cell. In addition to in vitro detection, AuNPs can be applied to SERS-based in vivo tumor targeting and detection. Qian et al. conjugated AuNPs with the ScFv antibody and injected them into nude mice bearing human head and neck tumors for in vivo tumor targeting and SERS spectroscopy [77], as shown in Figure 2.9. The signal intensities indicated 
significant differences between the targeted and non-targeted nanoparticles in the tumor, whereas the SERS signals from nonspecific liver uptake were similar. Therefore, $\mathrm{ScFv}$ conjugated AuNPs are able to target EGFR positive tumors in vivo.
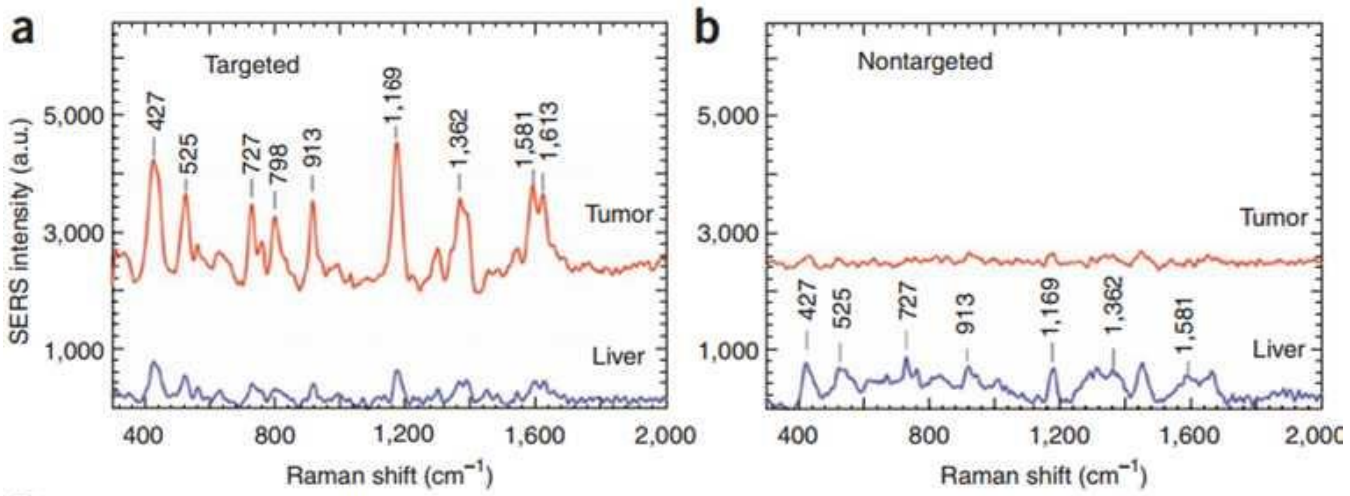

C
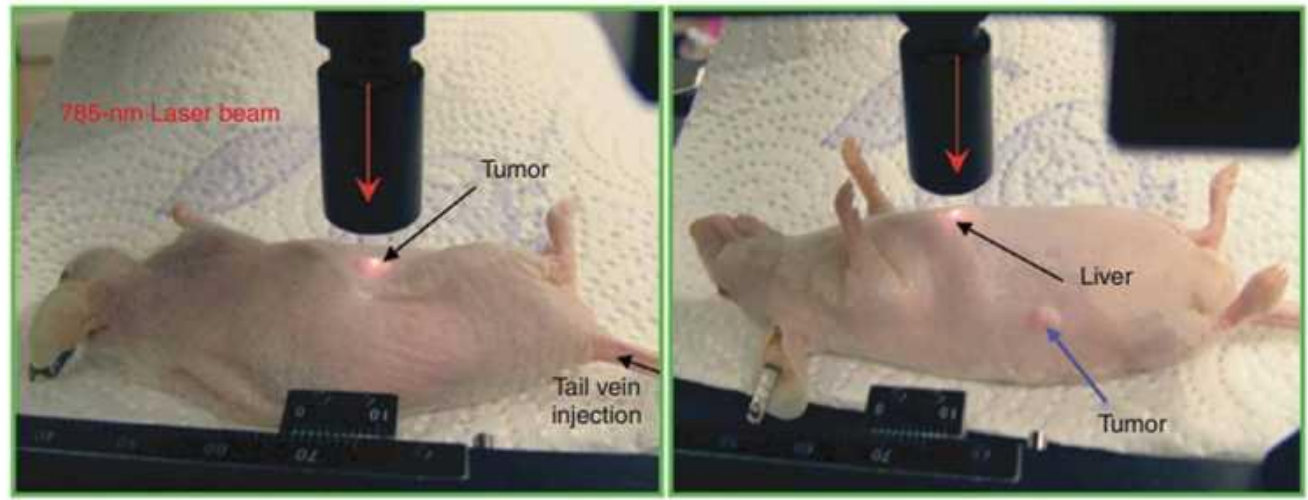

Figure 2.9 In vivo cancer targeting and SERS detection using ScFv antibody conjugated AuNPs that recognize the tumor biomarker EGFR [77].

The literature review presented here clearly shows that AuNPs with different sizes and shapes have great potential for use in biological applications. Owing to AuNPs' potential for translation into clinical phase testing, this research field will continue to grow rapidly. Therefore, my work provides a strong 
foundation and guidelines for optimizing the preparation of bio-conjugated AuNPs formulations for bio-imaging and bio-sensing applications. 


\section{Chapter 3 In Vitro Toxicity Studies of AuNRs formulations Coated with Bio-functional Thiol-PEG Molecules and Pluronic Block Copolymers}

\subsection{Introduction}

Over the past few years, the seed-mediated growth method proposed by Murphy and El-Sayed's group has been commonly used for synthesizing AuNRs formulations $[18,19,78]$. Cetyltrimethylammonium bromide (CTAB) molecules are used as structure directing agents in aiding the formation of gold rod-like particle in the aqueous medium. The issue with CTAB, however, is that it forms a tightly bound cationic bilayer on AuNRs surface with the cationic trimethylammonium head group exposed to the external environment. The presence of CTAB on the AuNRs surface poses a threat to many biological systems as they are toxic to cells and tissues. As a result, CTAB-coated AuNRs are not suitable to be used for biomedical applications $[79,80]$. CTAB can be partially removed from the Au NRs surface by centrifugation but most of it will remain on the particle surface and continue to show toxicity to cells at this stage. On the other hand, repeated centrifugations will result in AuNRs becoming structurally unstable and causing them to aggregate and precipitate in solution. Also, CTAB-coated AuNRs are not suitable for in vitro and in vivo applications because they do 
not allow antibodies or antigens to be linked to their surface for targeted delivery and imaging $[75,81]$. More importantly, one is not able to use CTAB-coated AuNRs as carrier for drug delivery of water insoluble anti-cancer agents such as doxorubicin and paclitaxel to the cancerous area since their surface is hydrophilic and positively charge [82, 83]. Therefore, a surface functionalization platform is needed to engineer AuNRs surface with biocompatible polymer-coating for reducing their cytotoxicity while maintaining colloidal stability and allowing them to be conjugated for biomedical applications. Bio functional thiol-Poly(ethylene glycol) (PEG-SH) molecules and Pluronic block copolymers (PEO-PPO-PEO) are commonly used for preparing non-ionic polymer encapsulated AuNRs with a stealth property for in vivo study [84-87]. It is worth noting that these PEG polymers can even be modified with additional functional groups such as carboxyl and amino group for conjugation of targeting ligands. It is documented that the CTAB bilayers on AuNRs surface can be removed and replaced with PEG-SH molecules through the chemisorption process between the thiol moiety and the gold particle surface $[88,89]$. Pluronic is a type of commercially available triblock copolymer with a structure of hydrophobic segment of poly (propylene oxide) (PPO) polymer sandwiched between two hydrophilic segments of PEO. In our previous study, we found that the hydrophobic PPO segment from the Pluronic block copolymer is able to bind to the hydrophobic 
part of CTAB molecules on AuNRs and form stable CTAB-polymer complexes $[90,91]$.

Upon functionalizing AuNRs with either PEG-SH or PEO-PPO-PEO molecules, many physiochemical property of AuNPs formulation will be affected and this may impact their applications in sensing, imaging and targeted delivery. Thus, it is essential for the nanoparticle community to understand the effects of functionalizing PEG-SH or PEO-PPO-PEO molecules on the AuNRs surface and their corresponding impact to the biological systems. In this work, we systematically study the cytotoxicity, colloidal stability and optical property of AuNRs before and after functionalizing them with PEG-SH and PEO-PPO-PEO molecules. These AuNRs formulations were characterized by using UV-vis spectroscopy, TEM, cell viability assay, DLS, and dark-field imaging microscopy. Non-specific uptake of these AuNRs by cells was also studied under dark-field microscopy. Our work demonstrates that the coating of PEG-SH or PEO-PPO-PEO molecules on AuNRs surface have significantly improved the colloidal and optical stability of the gold nanoformulation. No aggregation is found even after a few weeks of preparation. More importantly, the cell viability and dark-field imaging studies indicate that the AuNRs functionalized with PEG-SH or PEO-PPO-PEO molecules have minimal cytotoxicity and they can be used for long term in vitro and in vivo imaging study. 


\subsection{Experimental}

\subsubsection{Materials}

Hydrogen tetrachloroaurate (III) trihydrate $\left(\mathrm{HAuCl}_{4} \cdot 3 \mathrm{H}_{2} \mathrm{O}\right)$, cetylmethylammonium bromide $(\mathrm{CTAB})$, sodium borohydride $\left(\mathrm{NaBH}_{4}\right)$, silver nitrate $\left(\mathrm{AgNO}_{3}\right)$, L-ascorbic acid, trisodium citrate $\left(\mathrm{Na}_{3} \mathrm{C}_{6} \mathrm{H}_{5} \mathrm{O}_{7}\right)$, Pluronic F127 and cell counting kit (CCK8) were purchased from Sigma-Aldrich. PEG-SH ( $\left.\mathrm{CH}_{3} \mathrm{O}-\mathrm{PEG}-\mathrm{SH}\right)$ was purchased from Rapp Polymere. Dulbecco's modified Eagles's medium (DMEM) and 1x phosphate buffer sulphate (PBS) were prepared in-house. Fetal bovine serum (FBS) and penicillin/streptomycin (Pen Strep) were purchased from Gibco®. Clean-mount solution to fix glass cover slip over 8-chamber slides was purchased from Electron Microscopy Sciences.

\subsubsection{AuNRs synthesis and characterization}

Synthesis of AuNRs was adapted from Nikhoobakt et al [18]. Firstly, $5 \mathrm{~mL}$ of $0.5 \mathrm{mM} \mathrm{HAuCl}{ }_{4}$ was added to $5 \mathrm{~mL}$ of $200 \mathrm{mM} \mathrm{CTAB}$ to obtain an amber colored solution. $600 \mu \mathrm{l}$ of $10 \mathrm{mM} \mathrm{NaBH}_{4}$ were then added to the solution and stirred vigorously for a minute. A light brown seed solution was obtained. AuNRs were synthesized from seed mediated method and $5 \mathrm{~mL}$ of $1 \mathrm{mM}$ $\mathrm{HAuCl}_{4}$ were added to $5 \mathrm{~mL}$ of $200 \mathrm{mM} \mathrm{CTAB}$ and stirred. $350 \mu \mathrm{l}$ of $4 \mathrm{mM}$ $\mathrm{AgNO}_{3}$ were subsequently added. $70 \mu \mathrm{l}$ of $78.8 \mathrm{mM}$ of L-ascorbic acid were added and a colourless solution was formed. $18 \mu \mathrm{L}$ of the seed solution were injected into the growth solution and left to form AuNRs for an hour at room 
temperature. The AuNRs solution was centrifuged at 10,000 rpm for 10 minutes and suspended in water. This washing step was repeated for 3 times to remove excess CTAB. For AuNRs encapsulation, after the third time centrifugation, the supernatant was taken out and the remaining AuNRs were lefted as a pellet in the centrifuge tube without suspending them in water.

\subsubsection{TEM}

Transmission electron microscope (TEM) images to characterize the shapes and sizes of the AuNPs were taken with JEOL JEM-1010 operating at accelerating voltage of $80 \mathrm{kV}$. The specimens were prepared on 200 mesh nickel-coated TEM grids.

\subsubsection{UV-vis Absorption}

UV-vis absorption spectra were obtained with Hitachi U-2900 with a double-beam optical system and spectral bandpass of $1.5 \mathrm{~nm}$ over the spectrophotometric range of $400 \mathrm{~nm}$ to $1100 \mathrm{~nm}$. The specimen was placed in a quartz cuvette and deionized water was used as a reference.

\subsubsection{Functionalization of AuNRs}

$1 \mathrm{~mL}$ of Pluronic F127 or PEG-SH solution of various concentrations (10 nM, $100 \mathrm{nM}, 1 \mu \mathrm{M}, 10 \mu \mathrm{M}, 100 \mu \mathrm{M}$ and $1 \mathrm{mM}$ ) was added to AuNRs pellet. The resultant solutions were left to be incubated for 1 hour and then centrifuged once to remove excess Pluronic or PEG-SH solution. The functionalized AuNRs were then resuspended in water. The concentrations of the AuNRs solutions were fixed at the optical density of 1.5 for our studies. 


\subsubsection{Cell culture and cell viability}

Oral squamous cell carcinoma (OSCC) cell line was cultured in DMEM containing $10 \%$ FBS with Pen Strep. All cultures were maintained at $37^{\circ} \mathrm{C}$ with $5 \% \mathrm{CO}_{2} .5,000$ cells were seeded in a 96-well plate for 24 hours before loading each well with $10 \mu \mathrm{L}$ of AuNRs solution (Concentrations of all the solutions were fixed at the optical density of 1.5 with a UV-vis spectrophotometer). After further incubating the cells for 24 hours, $10 \mu \mathrm{L}$ of CCK8 were added to each well and allowed to incubate for 4 hours in dark at $37^{\circ} \mathrm{C}$ with $5 \%(\mathrm{v} / \mathrm{v}) \mathrm{CO}_{2}$. The cell population absorbance was performed with the SpectraMax 384 Plus spectral analyzer. The absorbance from the tetrazolium dye in CCK8 was measured at $450 \mathrm{~nm}$ excitation.

\subsubsection{In vitro dark-field imaging study}

5,000 cells suspended in media were seeded in each well of the 8-well chamber glass slide and allowed to be confluent, after which the media was then removed, the slide was rinsed with PBS and then the media was replenished in the wells. The corresponding synthesized substances were loaded and allowed to incubate for four hours at room temperature and pressure in dark. The media and synthesized substances were removed and rinsed with PBS again, and the cells were fixed with $4 \%$ paraformaldehyde for 10 minutes. Thereafter, the $4 \%$ paraformaldehyde was removed and the cells were rinsed with PBS. The well was removed and a cover slip was fastened with a layer of clean-mount on the slide. Dark-field imaging was performed 
with Nikon Eclipse $80 \mathrm{i}$ at 100x magnification. Hyperspectral Imaging technology was specifically designed to provide quantitative spectral analysis of nanoscale materials imaged with the dark-field-based microscope system. When observed via image analysis software, each pixel of a hyperspectral image provides the complete spectral response of that pixel's spatial area within the visible and near IR spectral range. At 100x magnification, a hyperspectral image may contain as many as 700,000 pixels as small as 128 $\mathrm{nm}$ each. This spectral data is recorded at approximately $2 \mathrm{~nm}$ of spectral resolution in the visible and near IR range, enabling minute spectral differences to be measured from pixel to pixel within the image.

\subsection{Results and Discussions}

\subsubsection{Stability of CTAB Stabilized AuNRs}

In this work, we used seed-mediated approach to synthesize AuNRs particle with longitudinal SPR at $750 \mathrm{~nm}$. During the formation of AuNRs, the CTAB molecule's head group preferentially binds to specific crystallographic faces of gold, thereby directing the gold atoms to deposit on selective faces of gold and attaining anistropic nanoparticles in the solution medium $[18,92,93]$. In this process, CTAB forms a tightly bound cationic bilayer on AuNRs surface resulting in $\mathrm{CTAB}$-coated particle, which are known to be cytotoxic to cells and tissues. We have found that a major fraction of CTAB molecules can be removed from the gold particle surface by multiple centrifugation steps. However, the washing steps will also affect the stability of the AuNRs and 
cause them to aggregate into precipitates in the solution. This is because CTAB molecules are known to serve as surfactants for passivating the particles surface and maintaining the colloidal stability of the particles. However, the disruption and removal of CTAB from the gold particles surface will result in the particles having large attractive forces between one another and causing them to aggregate into larger clusters. For a better understanding of this process, UV-vis absorption spectra were measured for AuNRs formulations before and after different treatments of washing steps. Figure 3.1

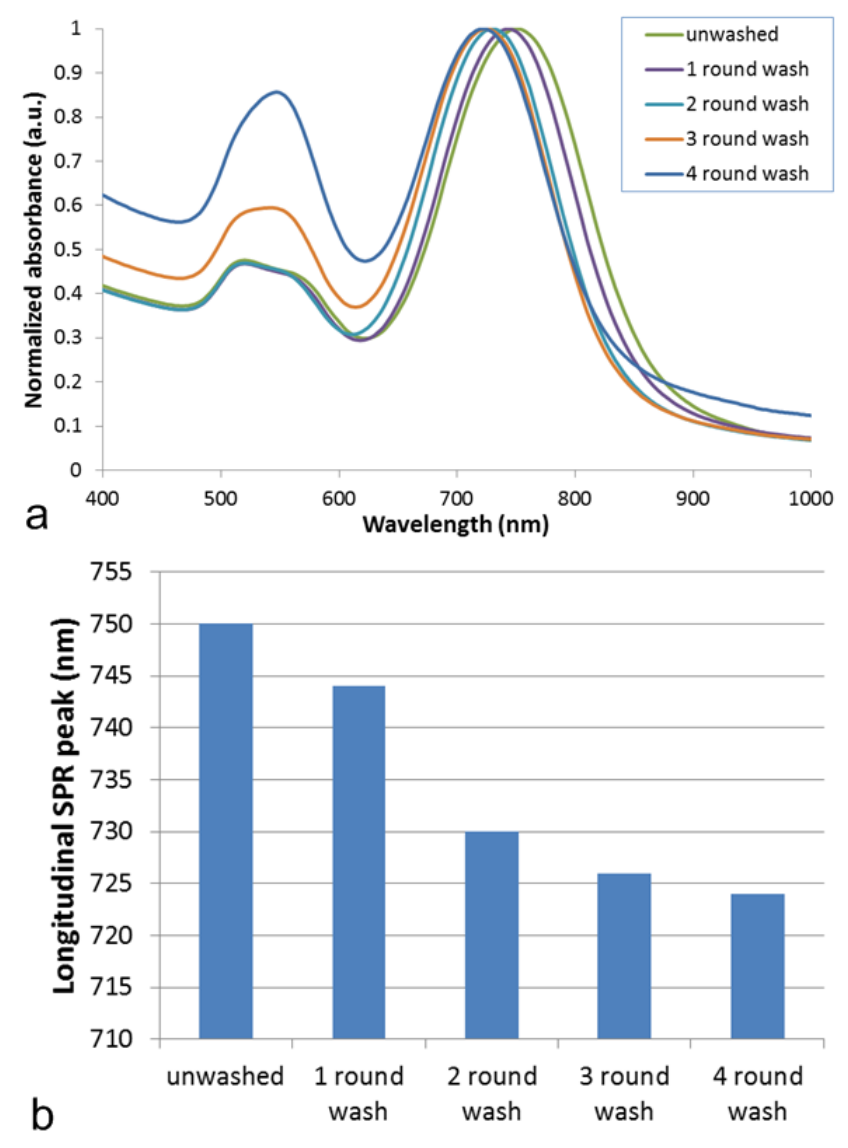

Figure 3.1 (a) Normalized UV-vis absorption spectra of as-synthesized AuNRs and AuNRs after different rounds of washing by centrifugation. (b) The longitudinal SPR absorption wavelength for different washing rounds. 
(a) and (b) show the normalized absorption spectra of AuNRs at different stages of washing treatments. Upon comparing the absorption spectrum of as-synthesized AuNRs to the treated AuNRs, it was observed that every round of washing caused a slight blue shift of the longitudinal SPR peak and an increase in the intensity of the transversal SPR peak starting from the third round of washing treatment. As the longitudinal SPR peak is determined by the aspect ratio of the AuNRs, the blue shift of this peak indicates that the aspect ratio of AuNRs decreases as the nanoparticles start to form large aggregates in the solution. To determine the morphology of AuNRs particle at various stages, we performed TEM analysis on both as-synthesized AuNRs and those from after the fourth round of washing treatment. The TEM image in Figure 3.2 (a) shows the as-synthesized AuNRs sample and more than $90 \%$ of the particles are rod-like in dimension, while Figure 3.2 (b) indicates that a large fraction of AuNRs formed larger spherical aggregates, accounting for rod-like particles less than $50 \%$ observed from the sample.

\subsubsection{PEG-SH or Pluronic Stabilized AuNRs}

To improve the biocompatibility of AuNRs for biological applications, we functionalized the particles with either PEG-SH or PEO-PPO-PEO molecules. Hydrophobic PPO chains of Pluronic copolymer are able to bind with the hydrophobic tails of $\mathrm{CTAB}$ molecules, leading to the passivation of the CTAB-coated AuNRs surface with PEO-PPO-PEO molecules. The hydrophilic PEO chains of the copolymer are then favorably interacting in the 


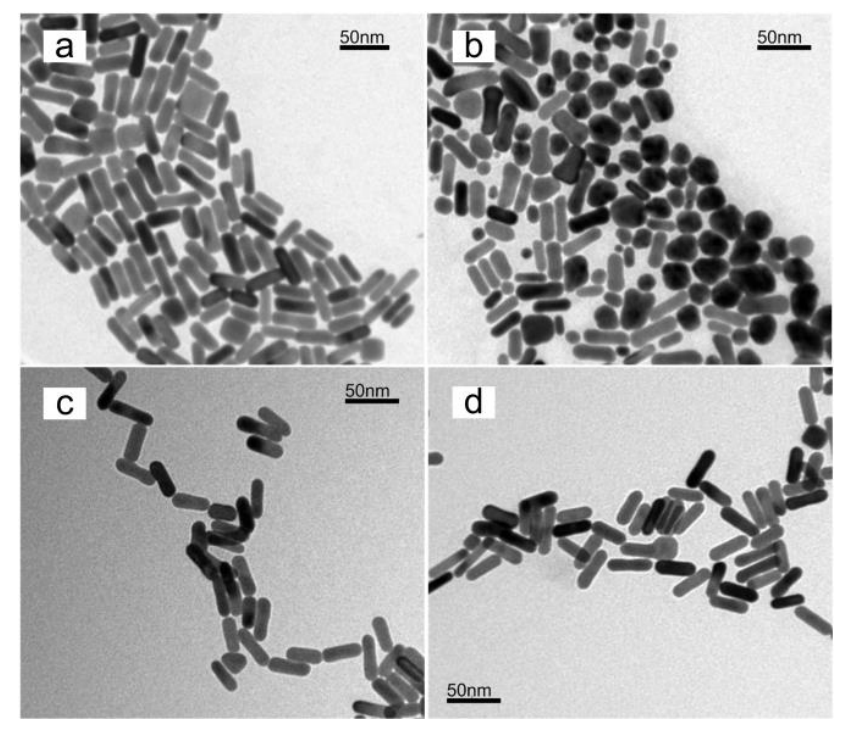

Figure 3.2 TEM images of (a) as-synthesized AuNRs, (b) AuNRs after 4 time centrifugation wash, (c) PEGylated AuNRs and (d) PEO-PPO-PEO encapsulated AuNRs.

aqueous phase thereby maintaining the overall colloidal solubility of the Pluronic passivated AuNRs. Pluronic F127 was chosen as it has comparable long PPO and PEO chain. Therefore, the advantages of these two block copolymers are combined to provide both better surface passivation on the particles and better colloidal stability [94-96]. PEG-SH and Pluronic-encapsulated AuNRs were prepared by retrieving particles after the third round of the washing treatment. Figure 3.2(c) and (d) show the TEM images of AuNRs functionalized with PEG-SH and PEO-PPO-PEO molecules, respectively. In comparison to Figure 3.2(a), one can observe that the overall size and shape of AuNRs functionalized with either PEG-SH or PEO-PPO-PEO molecules remained the same, thus demonstrating that these 
two polymer molecules are suitable to be utilized in engineering the particle surface for maintaining the optical and colloidal stability of AuNRs.

\subsubsection{Cell Viability}

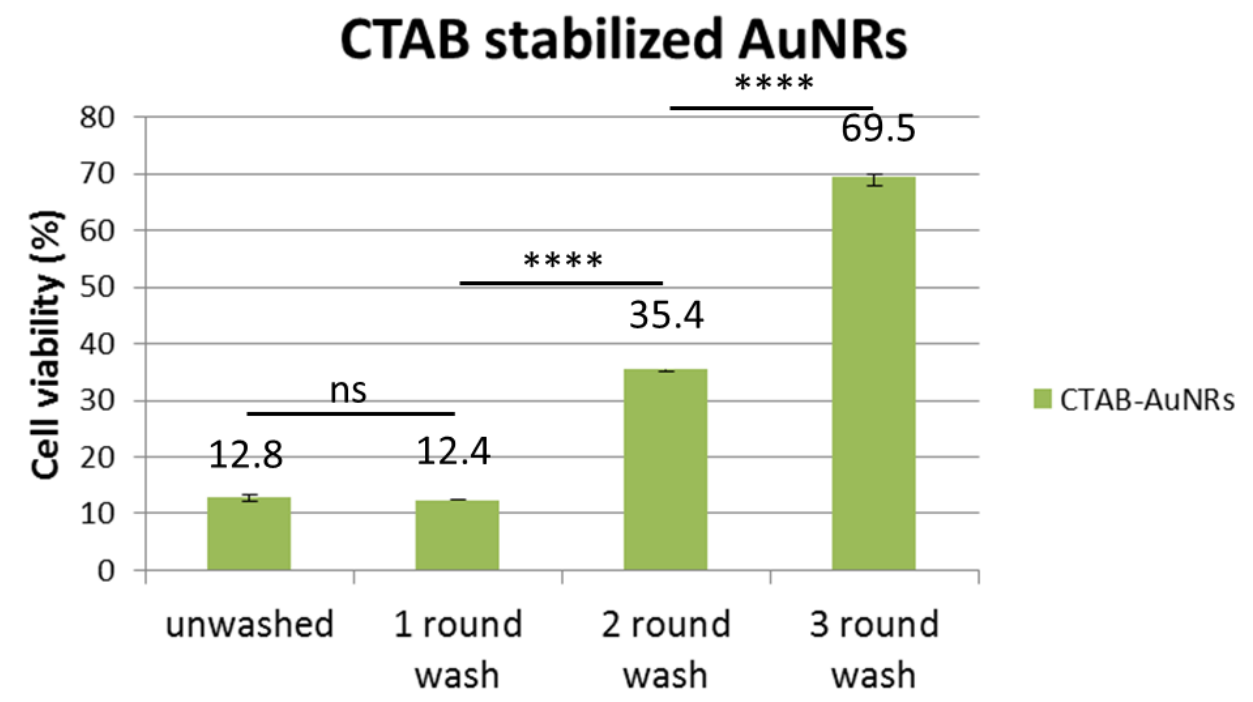

Figure 3.3 Relative cell viability of OSCC cells treated with as-synthesized AuNRs and AuNRs after different rounds of washing treatments for 24 hours post-treatment.

To systematically study the cytotoxicity of these functionalized AuNRs formulations, different concentrations of PEG-SH and PEO-PPO-PEO were used to react with $\mathrm{CTAB}-$ coated AuNRs and thereby producing AuNRs formulations with different surface coverage of PEG-SH and PEO-PPO-PEO molecules. The cytotoxicity between the as-synthesized AuNRs and AuNRs after different rounds of washing treatment was also compared. As shown in Figure 3.3, since as-synthesized AuNRs formulation have excess CTAB molecules not only on the particle surface but also in the solution, this 
formulation shows high toxicity to the cells and cell viability of OSCC cells is maintained around $10 \%$ at 24 hours post-treatment. Upon treating the OSCC cells with AuNRs after three rounds of washing, we are able to observe a significant increase in the cell viability to as high as $70 \%$. This demonstrates that the toxicity of the formulation was drastically reduced by removing CTAB surfactants from the particle suspension. However, further washing treatment to the AuNRs is infeasible because the particles will become unstable in the absence of CTAB surfactants in the suspension. The cell viability of AuNRs functionalized by different concentrations of PEG-SH or PEO-PPO-PEO surfactants is shown in Figure 3.4. It is clear that functionalizing PEG-SH and PEO-PPO-PEO molecules to AuNRs surface can dramatically reduce the formulation cytotoxicity and this can be observed from the cell viability assay where the percentage is maintained up to nearly $90 \%$ when concentrations ranging from $10 \mathrm{~nm}$ to $1 \mathrm{mM}$ of both PEG-SH and PEO-PPO-PEO molecules are used in the experiment. However, we observed that there is an optimum concentration of PEG-SH and PEO-PPO-PEO molecules that can be used for synthesizing AuNRs with the lowest cytotoxicity. We found that the use of $1 \mu \mathrm{M}$ PEG-SH or $100 \mu \mathrm{M}$ PEO-PPO-PEO to treat CTAB-coated AuNRs is able to produce highly biocompatible particles formulation for in vitro applications. Further reacting CTAB-coated AuNRs with higher concentration of PEG-SH and PEO-PPO-PEO molecules has resulted in decreased cell viability. This may b- 


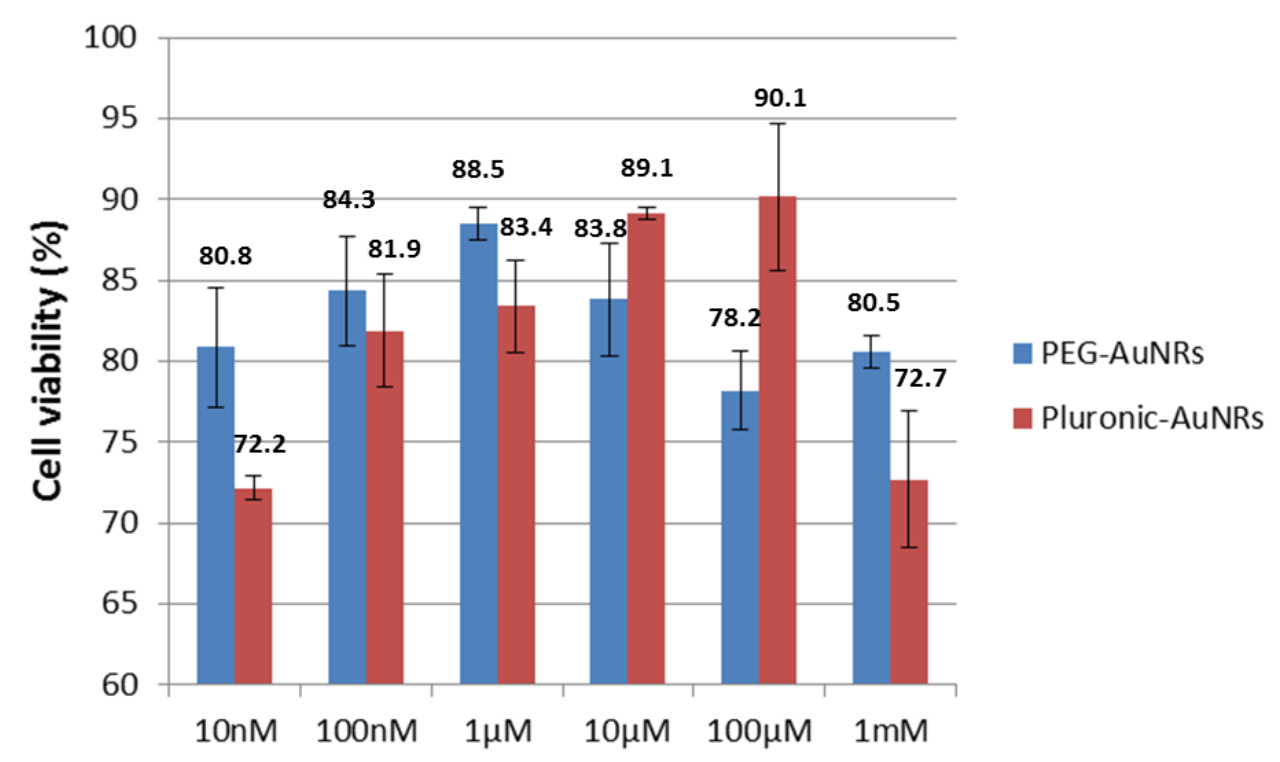

Figure 3.4 Cell viability of AuNRs encapsulated with different concentrations of PEG-SH or Pluronic triblock copolymer. The concentration of PEG-SH and Pluronic adopted for encapsulation spans from $10 \mathrm{nM}$ to $1 \mathrm{mM}$.

e resulted from the formation of a thicker coating layer on the AuNRs surface [97]. Many groups have reported that the encapsulation of AuNRs with other polymer coating to improve the biocompatibility of the rod nanoparticle formulations. For example, Alkilany et al demonstrated the use of polyacrylic acid (PAA) and poly(allylamine) hydrochloride (PAH) to coat AuNRs surface. The coating was performed on as-synthesized CTAB-coated AuNRs after centrifugation washing treatment [80]. The CTAB-coated AuNRs solution was found to reduce cell viability by $30 \%$, while both PAA-coated AuNRs and PAH-PAA-coated AuNRs were found to be non-toxic with cell viability $\sim 90 \%$. Takuro et al demonstrated AuNRs with $\sim 90 \%$ cell viability show stealth character in vivo using mice as a model [84]. It was also found 
that the effective hydrodynamic diameters of PAA-coated AuNRs and PAH-PAA-coated AuNRs increase from 20 nm (CTAB-coated AuNRs) to 25 $\mathrm{nm}$ and $30 \mathrm{~nm}$, respectively, indicating the successful coating of polymer layer on the AuNRs surface. Wang et al also reported the cytotoxicity of AuNRs under different conditions [98]. They found that the as-synthesized AuNRs formulation was highly toxic and a very low cell viability result $(\sim 10 \%)$ was observed for this formulation. However, after 3 rounds of centrifugation washing, the cytotoxicity of CTAB-coated AuNRs was found to decrease but not to the extent that they can be used for biological studies since they are still cytotoxic. A similar observation was made in our study.

\subsubsection{Mean Hydrodynamic Diameters of AuNRs}

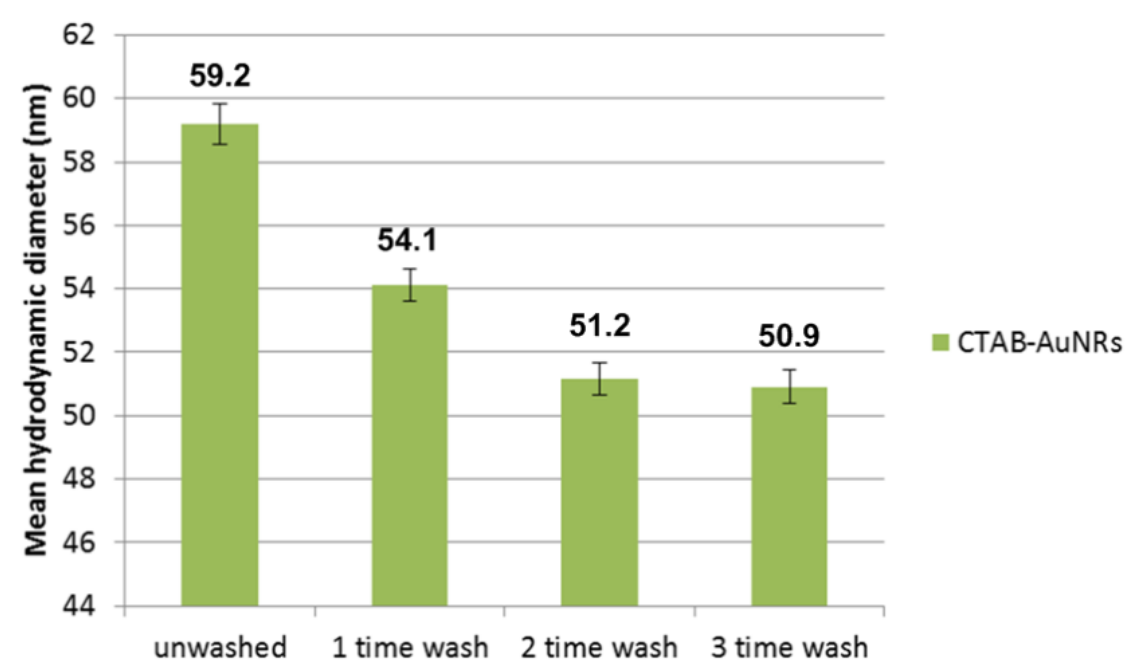

Figure 3.5 Mean hydrodynamic diameters of as-synthesized AuNRs (unwashed) and AuNRs after different rounds of centrifugation wash. Hydrodynamic diameter infers the diameter of the outermost encapsulation layer on a particle. 


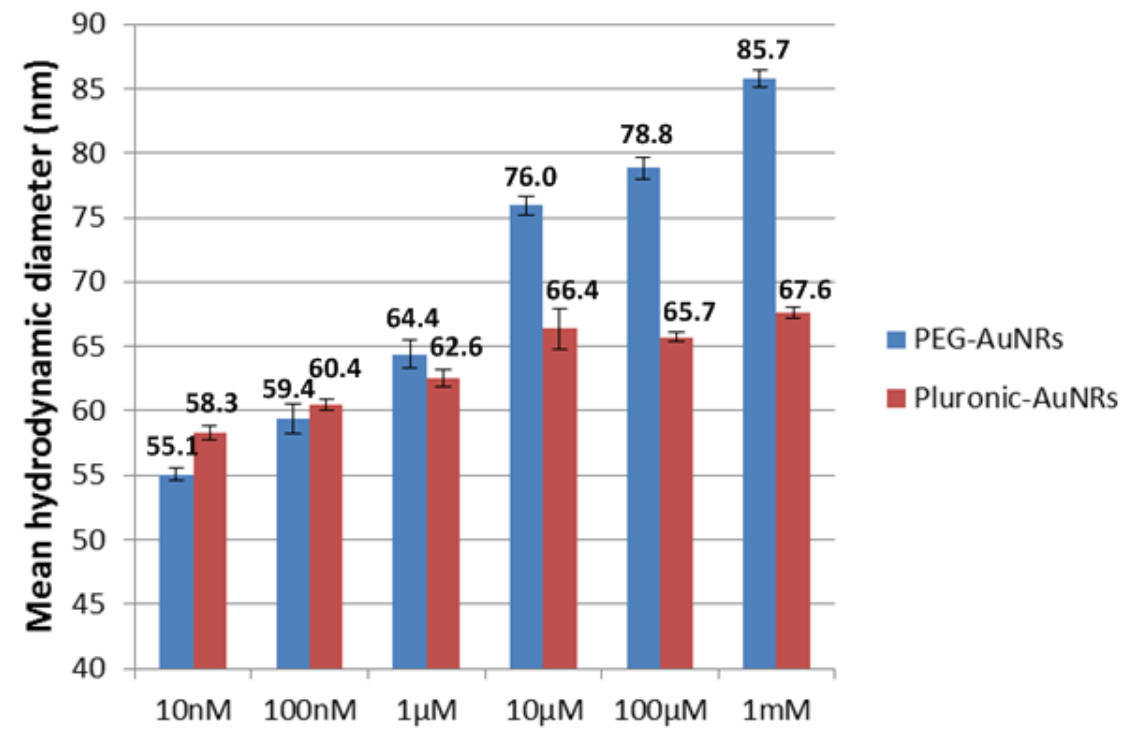

Figure 3.6 Mean hydrodynamic diameter of AuNRs encapsulated with different concentrations of PEG-SH or Pluronic.

DLS experiments were performed in our study to determine the hydrodynamic diameter and the colloidal stability of the prepared AuNR formulations. Figure 3.5 shows the mean hydrodynamic diameter of CTAB-coated AuNRs before and after different rounds of washing treatment. A slight decrease ( 6 to $7 \mathrm{~nm}$ ) in the overall hydrodynamic diameter of the nanoparticles formulation was observed after an additional round of washing and this indicates that a fraction of CTAB molecules was removed from AuNRs surface during every round of centrifugation washing. In general, we observed that AuNRs became less stable in aqueous phase and formed aggregates after 2 to 3 rounds of washing. In Figure 3.6, we have observed that the mean hydrodynamic diameter of AuNRs increases slightly when the concentration of PEO-PPO-PEO and PEG-SH increases in the reaction mixture. The 
hydrodynamic sizes of the PEG-SH or PEO-PPO-PEO functionalized AuNRs are found to be larger than those of AuNRs determined by TEM analysis and this is mainly due to the solvated polymer layers of PEO-PPO-PEO or PEG-SH on the AuNRs surface. In our study, only AuNRs that have gone through three rounds of washing treatment were used for functionalization of polymer molecules. The hydrodynamic diameter of AuNRs was determined to be 55 and $58 \mathrm{~nm}$ after passivating their surface with PEO-PPO-PEO and PEG-SH molecules.

\subsubsection{Dark-field Imaging}

For in vitro imaging study, OSCC cells were treated with AuNRs functionalized with either PEG-SH or PEO-PPO-PEO molecules for evaluating their biocompatibility. In accordance with the cell viability results shown in Figure 3.4, three concentrations of PEG-SH $(10 \mathrm{nM}, 1 \mu \mathrm{M}, 1 \mathrm{mM})$ and Pluronic (10 nM, $100 \mu \mathrm{M}, 1 \mathrm{mM})$ were used to passivate AuNRs and these formulations were employed for in vitro dark-field imaging. Figure 3.7 shows dark-field images of OSCC cells with the encapsulated AuNRs synthesized in this study. The bright red and orange scattered spots located within the cells suggesting that the AuNRs were internalized into the cells through non-specific cellular uptake since no biomolecules were attached to our gold formulations. Huang et al performed a similar experiment where AuNRs were also observed to be internalized by malignant oral epithelial cell lines and the extinction spectra analysis confirmed that the scattering colors 
within the cells was resulting from nanoparticles [30]. Thus, the coupling of the inherent scattering property of AuNRs with the use of polymer-based encapsulation further facilitates the use of AuNRs as biocompatible in vivo probes.

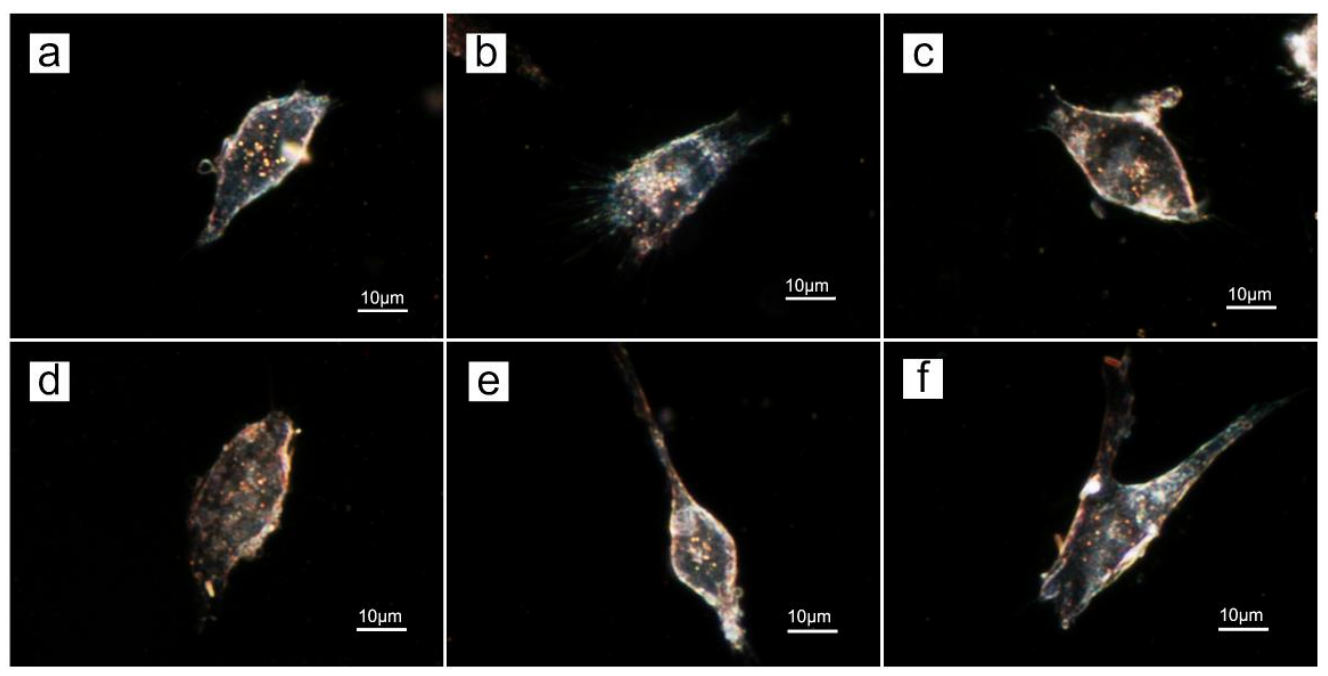

Figure 3.7 Dark-field images of OSCC cells with AuNRs encapsulated with

(a) $10 \mathrm{nM}$, (b) $1 \mu \mathrm{M}$, (c) $1 \mathrm{mM}$ of PEG-SH and (d) $10 \mathrm{nM}$, (e) $100 \mu \mathrm{M}$ (f) 1 $\mathrm{mM}$ of Pluronic F127.

\subsection{Summary}

In this work, we studied the cytotoxicity, colloidal stability and optical property of AuNRs before and after functionalizing them with PEG-SH and PEO-PPO-PEO molecules. The as-synthesized AuNR surfaces are functionalized with $\mathrm{CTAB}$ molecules and such a formulation is highly toxic and not suitable to be used for any biological applications. To employ the AuNRs for biological studies, AuNRs surface need to be passivated with a 
biocompatible polymer coating. We have found that encapsulating AuNRs with the PEG-SH or PEO-PPO-PEO molecules will produce excellent colloidal stable and biocompatible AuNRs formulations and they can be readily used for dark-field imaging of cancer cells. We believe that this work will provide useful insight for developing new protocols for preparing biocompatible AuNRs for applications ranging from cell imaging to targeted drug delivery in vivo. 


\section{Chapter 4 Biocompatible Probes Based on Pluronic Triblock Copolymer Encapsulated AuNRs}

\subsection{Introduction}

Over the past decade, various methods for the synthesis of AuNPs of various shapes and sizes have been reported. On one side, the SPR strongly depends on the shape and size of AuNPs and can be easily tuned by changing its dimensions. For isotropic nanoparticles such as nanospheres, the SPR absorption peak is proportional to their diameter. In the case of AuNRs with an anisotropic shape, the SPR absorption peak splits into a transverse mode and a longitudinal mode corresponding to the diameter and length of the rod, respectively. On the other side, for applications such as optical coherence tomography, photothermal therapy and photodynamic therapy, the SPR absorption peaks of nanoparticles can be tuned from 650 to $900 \mathrm{~nm}$ to optimize the light for tissue depth penetration[99]. Furthermore, cellular uptake of particulates can be up to $100 \mathrm{~nm}$, and the optimum size is around 50 $\mathrm{nm}[100]$. Considering all aforementioned facts, AuNRs are intriguing among all possible nanoparticle shapes as they have a high SPR tunability while maintaining a relatively small size compared to other nanoparticles.

AuNRs are synthesized with the help of CTAB as a structure inducing agent. However, it is suggested that CTAB forms a tightly bound cationic bilayer on 
AuNRs with the cationic trimethylammonium head group exposed to the aqueous media. CTAB exposure induces cytotoxicity, thus limiting the use of AuNRs in biomedical applications [79, 80]. Reducing the cytotoxicity and enhancing the biocompatibility of AuNRs remained a great challenge in the last few years. Many methods were reported to reduce the cytotoxicity of the CTAB-coated AuNRs, such as encapsulation of AuNRs with mPEG-SH (methoxy (polyethylene glycol)-thiol) [101], bovine serum albumin (BSA) and polyethylenimine (PEI) [102] for biological applications.

In this work, we propose the encapsulation of AuNRs with Pluronic, a non-ionic triblock polymer that can associate with CTAB and form stable CTAB-polymer complexes through hydrophobic interactions, and thus reduce the cytotoxicity. These encapsulated AuNRs are characterized by UV-vis absorption spectra analysis and TEM. Subsequently, cell viability of OSCC cell line that is treated with these AuNRs is studied. We also report the use of Pluronic-encapsulated AuNRs as a biocompatible optical probe with capability for in vitro dark-field imaging.

\subsection{Experimental}

\subsubsection{Materials}

Hydrogen tetrachloroaurate (III) trihdyrate $\left(\mathrm{HAuCl}_{4} \cdot 3 \mathrm{H}_{2} \mathrm{O}\right)$, cetylmethylammonium bromide (CTAB), sodium borohydride $\left(\mathrm{NH}_{4}\right)$, silver nitrate $\left(\mathrm{AgNO}_{3}\right)$, L-ascorbic acid, Pluronic F108 and F127, trisodium citrate 
$\left(\mathrm{Na}_{3} \mathrm{C}_{6} \mathrm{H}_{5} \mathrm{O}\right)$ and cell counting kit (CCK8) were purchased from Sigma-Aldrich. Dulbecco's modified Eagles's medium (DMEM) and 1x phosphate buffer sulphate (PBS) were prepared in-house. Fetal bovine serum (FBS) and penicillin/streptomycin (Pen Strep) were purchased from Gibco®. Clean-mount solution to fix glass cover slip over 8-chamber slides was purchased from Electron Microscopy Sciences.

\subsubsection{Gold Nanoparticle Seed Synthesis}

Synthesis of gold seeds was adapted from Nikhoobakt et al. [18]. Briefly, 5 $\mathrm{mL}$ of $0.5 \mathrm{mM} \mathrm{HAuCl}_{4}$ was added to $5 \mathrm{~mL}$ of $200 \mathrm{mM} \mathrm{CTAB}$ to obtain an amber coloured solution. $600 \mu \mathrm{L}$ of $10 \mathrm{mM} \mathrm{NaBH}_{4}$ was then added to the solution and stirred vigorously for a minute. A light brown solution was obtained.

\subsubsection{Encapsulation of AuNRs with Pluronic}

Pluronic solutions of corresponding types (F-108 and F-127) and concentrations $(3,5,10,20$ and $30 \mathrm{mg} / \mathrm{mL})$ were added to AuNRs solutions in one-to-one ratio and mixed. The resultant solutions were left to be incubated for 2 hours and then centrifuged to remove the excess Pluronic solutions. The encapsulated AuNRs were then resuspended in water. Concentrations of the AyNRs solutions were fixed at an optical density of 1.5 with a UV-vis spectrometer. 


\subsubsection{Dark-field Imaging}

Dark-field imaging was performed with Nikon Eclipse 80i at 100x magnification and local absorption spectra were analyzed with the Hyperspectral camera module.

\subsubsection{Differential Interference Contrast Microscopy}

Differential Interference Contrast (DIC) microscopy was performed with Nikon A1 Confocal Microscope at 20x magnification powered with $405 \mathrm{~nm}$ laser to image DAPI-stained nuclei of cells.

\subsubsection{Other Experiments}

Experimental of AuNRs synthesis, TEM, UV-vis absorption, cell culture and cell viability, and dark-field sample preparation are described in the experiments part in Chapter 3.

\subsection{Results and Discussions}

\subsubsection{AuNRs Synthesis and Encapsulation}

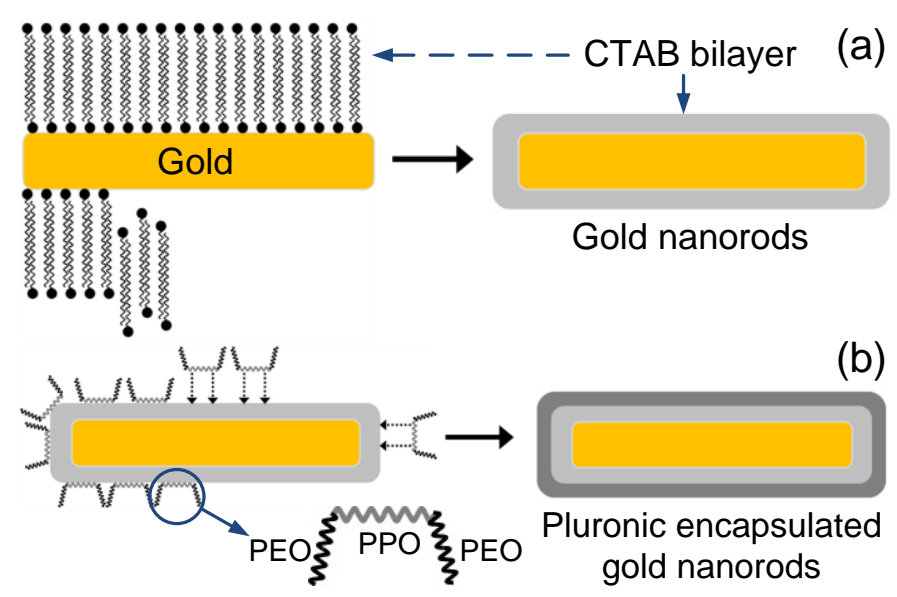

Figure 4.1 (a) CTAB (black squiggles) as a structure inducing agent during AuNRs formation as more gold ions (yellow blocks) are introduced. (b) The 
PPO block of Pluronic binds with hydrophobic tail of CTAB by hydrophobic interactions and thus encapsulates AuNRs.

\begin{tabular}{|c|c|c|c|c|c|c|}
\hline \multirow{2}{*}{ Seed vol. } & \multicolumn{2}{|c|}{200} & \multicolumn{2}{|c|}{300} & \multicolumn{2}{|c|}{350} \\
\hline & $\begin{array}{l}\text { TPR } \\
(\mathrm{nm})\end{array}$ & $\begin{array}{l}\text { LPR } \\
(\mathrm{nm})\end{array}$ & $\begin{array}{l}\text { TPR } \\
(\mathrm{nm})\end{array}$ & $\begin{array}{l}\text { LPR } \\
(\mathrm{nm})\end{array}$ & $\begin{array}{l}\text { TPR } \\
(\mathrm{nm})\end{array}$ & $\begin{array}{l}\text { LPR } \\
(\mathrm{nm})\end{array}$ \\
\hline 15 & \multicolumn{2}{|c|}{515,701} & \multicolumn{2}{|c|}{517,716} & \multicolumn{2}{|c|}{520,764} \\
\hline 18 & \multicolumn{2}{|c|}{513,696} & \multicolumn{2}{|c|}{515,718} & \multicolumn{2}{|c|}{530,777} \\
\hline 20 & \multicolumn{2}{|c|}{510,713} & \multicolumn{2}{|c|}{521,743} & \multicolumn{2}{|c|}{525,772} \\
\hline
\end{tabular}

Table 4.1 Table of transverse LSPR (TPR) and longitudinal LSPR (LPR) wavelength of AuNRs synthesized with varied $\mathrm{AgNO}_{3}$ and seed volumes. Environmental condition for each synthesis was kept consistent.

We optimized the reported seed-mediated growth method [18] to synthesize AuNRs with transverse SPR peak of $520 \mathrm{~nm}$ and longitudinal SPR peak ranging from $750 \mathrm{~nm}$ to $800 \mathrm{~nm}$ with aspect ratio 4 (as shown in Table 4.1). The AuNRs are grown from gold seeds and subsequent reduction of gold salt $\left(\mathrm{HAuCl}_{4}\right)$ with a weak reducing agent (ascorbic acid) in a micellar medium[103]. For the formation of AuNRs, CTAB is an important ingredient and it was reported that the CTAB head group can preferentially bind to the crystallographic faces of gold and act as a structure directing agent that "zips" up gold ions linearly to form the rod shape (Figure 4.1(a)) [93]. Pluronic is a commercially synthesized non-ionic triblock polymer of a hydrophobic core chain of poly (propylene oxide) (PPO) sandwiched at its ends by two hydrophilic chains of poly (ethylene oxide) (PEO). The hydrophobic PPO 
block of Pluronic can bind with the hydrophobic tail of CTAB and form stable CTAB-polymer complexes (Figure 4.1(b)) [90]. In comparison to CTAB, Pluronic presents higher biosafety since it has been approved by FDA for many years [104]. Two types of Pluronic, F-127 and F-108 were used to encapsulate AuNRs because they have longer PPO chains thus leading to better surface passivation and colloidal stability $[94,95]$.

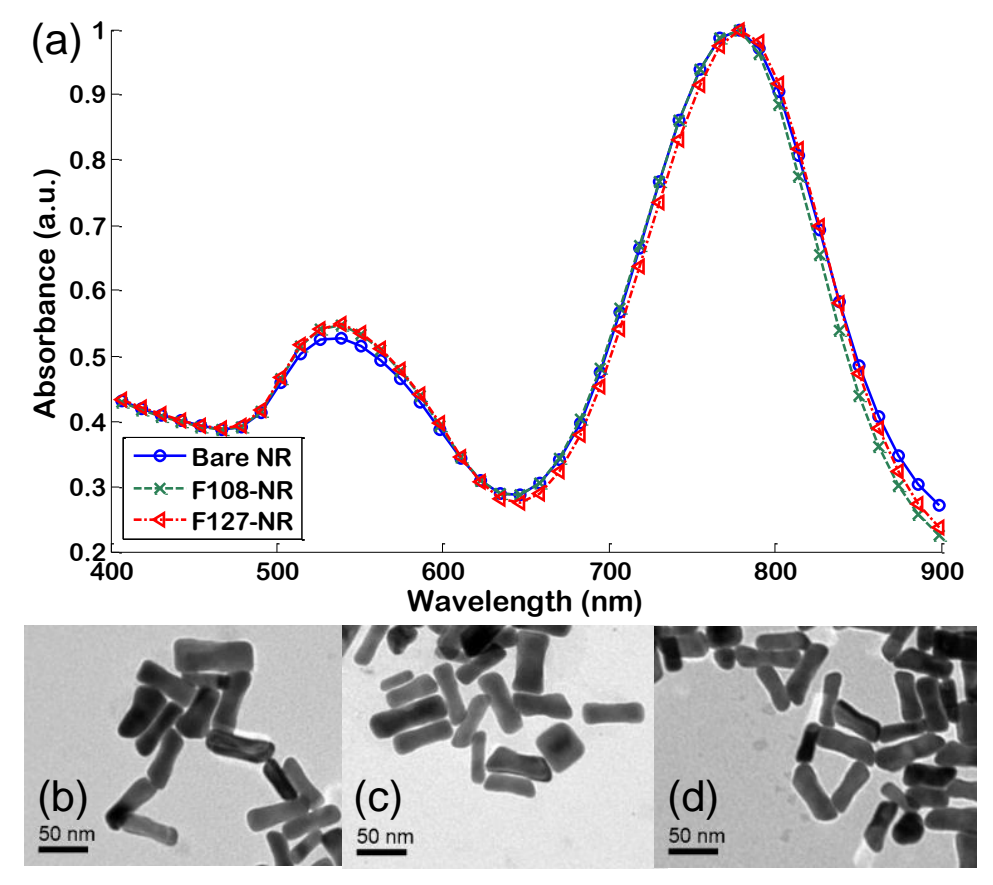

Figure 4.2 (a) UV-vis spectra of CTAB-coated AuNRs (Bare NR), Pluronic F-108 encapsulated AuNRs (F108-NR) and Pluronic F-127 encapsulated AuNRs (F127-NR). The UV-vis spectra were taken from AuNRs suspended in HPLC water. TEM images of (b) Bare AuNRs, (c) F108-AuNRs and (d) F127-AuNRs. (Concentration of Pluronic: 20mg/mL).

As shown in Figure 4.2(a), Pluronics-encapsulated AuNRs exhibited no observable change in the UV-vis absorption spectra compared to the 
CTAB-coated AuNRs. Since the transverse SPR and longitudinal SPR peaks are determined by the diameter and length of the nanorods, the UV-vis absorption spectra suggest that the encapsulation of Pluronic on AuNRs did not alter their dimensions. Furthermore, this was confirmed by the TEM images shown in Figure 4.2(b), (c) and (d), where no change in dimension was observed for AuNRs before and after the coating treatment with Pluronic. In addition, we also observed that passivation of Pluronic of the AuNRs surfaces can minimize the blue shifting of longitudinal SPR peak and suggests that they can stabilize AuNRs for long term applications. CTAB coated AuNRs exhibit a $20 \mathrm{~nm}$ blue shift while Pluronic-encapsulated AuNRs show only a $\sim 7 \mathrm{~nm}$ shift over 10 days, as shown in Figure 4.3.

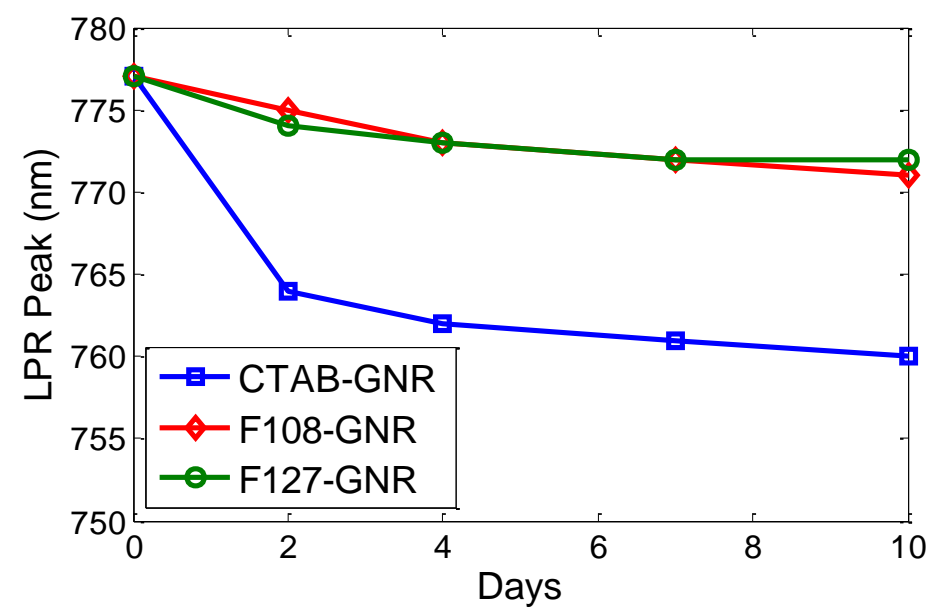

Figure 4.3 Blueshift variation trend with respect to the longitudinal SPR peak of the absorption spectra of CTAB-coated AuNRs (CTAB-GNR), F-108-coated AuNRs (F108-GNR) and F-127-coated AuNRs (F127-GNR) stored at room temperature over 10 days (The concentration of Pluronic is $20 \mathrm{mg} / \mathrm{mL})$. 


\subsubsection{Cell viability and DIC microscopy}

Cell viability tests were performed to evaluate the cytotoxicity of the Pluronic-encapsulated AuNRs using CCK assay with OSCC cell line. In this experiment, the CTAB-coated AuNRs were washed few times to remove excess unbounded $\mathrm{CTAB}$ before using them for the cell viability study. However, washing cannot remove the CTAB molecules bounded on the surface of AuNRs as indicated by relatively high cytotoxicity (70\% viability) as shown in Figure 4.4(a). On the contrary, AuNSs synthesized with trisodium citrate are non-toxic to the cells due to the citrate stabilization. In contrast, all the Pluronic-encapsulated AuNRs formulations maintained more than $80 \%$ cell viability, even at Pluronic concentration as low as $3 \mathrm{mg} / \mathrm{mL}$ (Figure 4.4(b)). This indicates that such Pluronic-encapsulated AuNRs can be used as efficient nano-probes at non-cytotoxic dosages. The cells morphology images from the cell viability study are shown in Figure 4.5 by using DIC microscopy. Figure 4.5(a) shows the morphology of cells treated with CTAB-coated AuNRs and their flattened structure reveal that most cells are non-viable. While in Figure 4.5(b), the rounded structure of cells shows large amount of cells are viable. The cell viability study and DIC images not only confirms the cytotoxicity of the CTAB-coated AuNRs, thereby justifying our approach of the Pluronic triblock copolymer layering, but also demonstrates the biocompatibility of the Pluronic-encapsulated AuNRs formulations and highlighting their potential for in vitro imaging. It is worth noting that the 
viability of the cells treated with the F108 and F127 Pluronic-Encapsulated AuNRs is more or less demonstrating the same biocompatibility.

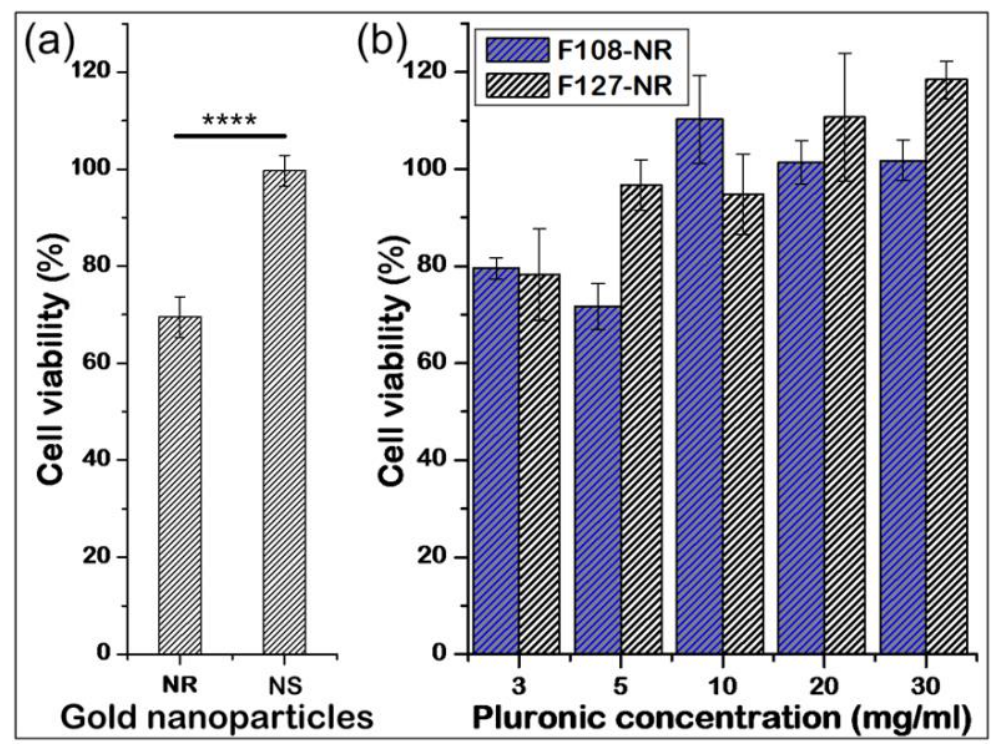

Figure 4.4 (a) Cell viability of bare AuNRs (NR) (after centrifugation) and AuNSs (NS), (b) Cell viability of Pluronic F-127 and F-108 encapsulated AuNRs with different Pluronic concentrations.

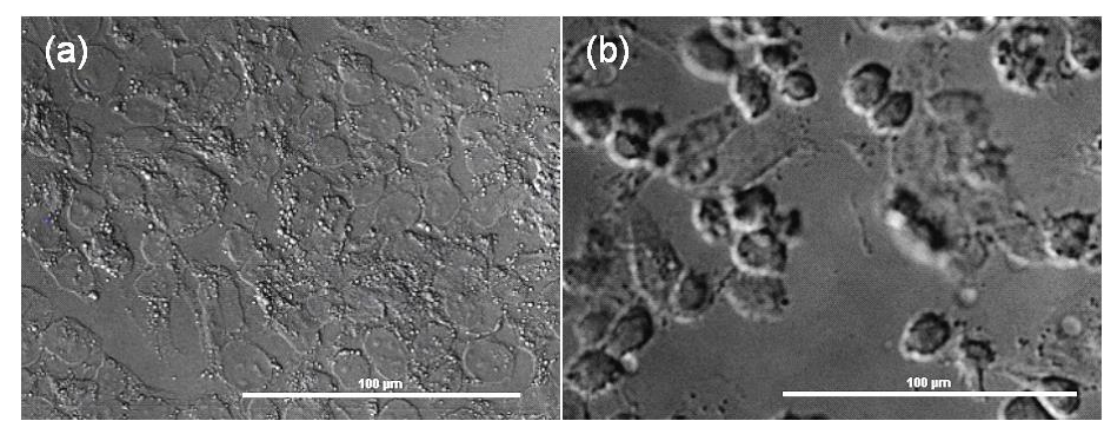

Figure 4.5 DIC microscopy images of a) OSCC cells treated with CTAB-coated AuNRs are shown to be non-viable (flattened structure), b) OSCC cells treated with AuNRs encapsulated with F127 (30mg/mL) (rounded structure), shows CTAB-coated AuNRs are highly toxic to the cells while Pluronic-encapsulated AuNRs show highly biocapatibility. (bar = 100um) 


\subsubsection{Dark-field Imaging}

The Pluronic-encapsulated AuNRs formulation was then used as scattering probes for cancer cells imaging. Previously, it was reported that nanoparticles encapsulated with Pluronics copolymers or PEG molecules were able to label the cells through non-specific uptake of the nanoparticles by the cells [105]. In this study, the uptake of Pluronic-encapsulated AuNRs by OSCC cells was investigated using dark-field imaging. Fig 3.6(a) shows the control image of the OSCC cells without the addition of AuNRs. In comparison, Figure 4.6(b) and (c) with yellow/orange scattering color dots within the cancer cells suggests that a large quantity of Pluronic-functionalized AuNRs are internalized though non-specific uptake. In addition to dark-field imaging, the local absorption spectra analysis was performed on the cancer cells treated with Pluronic-encapsulated AuNRs. We have measured the yellow and orange scattering color spots on the cancer cells using hyper-spectra imaging analysis. Transverse SPR and longitudinal SPR peaks observed from the measured hyper-spectra in Figure 4.6 (d) and (e) confirmed that the yellow/orange scattering color spots originated from the Pluronic-encapsulated AuNRs. It is worth noting that a blue shift was also observed for the SPR peaks from the hyper-spectra as compared to the UV-vis spectra in Figure 4.2 (a). This blue shift may be due to (i) a different refractive index of the medium that causes an SPR shift (UV-vis spectra were taken from AuNRs suspended in water while hyper-spectra were taken from AuNRs 
on dry glass slide in air) [106], and (ii) the differences in the orientation of the AuNRs within the cells.
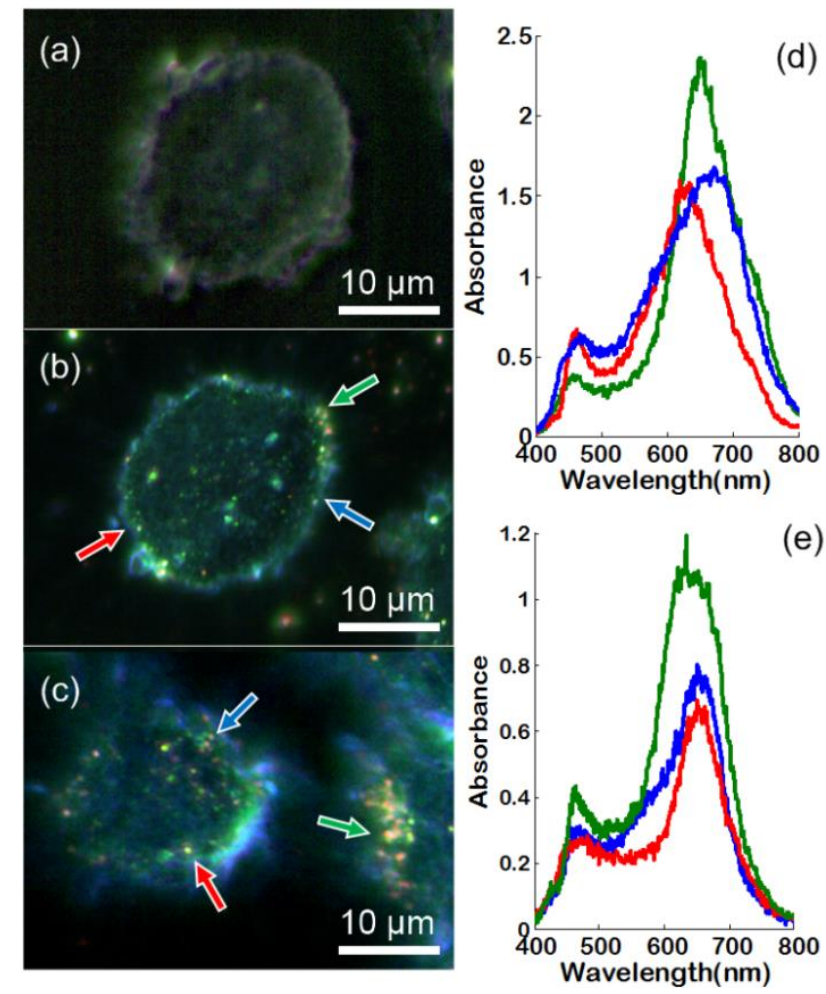

Figure 4.6 Dark-field images of OSCC cells (a) without AuNRs, (b) uptaking of Pluronic F-108 encapsulated AuNRs, and (c) uptaking of Pluronic F-127 encapsulated AuNRs. Hyperspectral absorption spectra of (d) F-127 encapsulated AuNRs (pointed out by arrows in Figure 4.6(b)) and (e) F-108 encapsulated AuNRs (pointed out by arrows in Figure 4.6(c)).

\subsection{Summary}

In this chapter, we have prepared AuNRs encapsulated with Pluronic F-108 and F-127 for in vitro dark-field imaging. UV-vis spectra determine that the layering of Pluronic surfactants on the AuNRs surface did not change the 
dimension in comparison to the CTAB-coated AuNRs. In addition, the layering of Pluronic can significantly improve the colloidal and optical stability of AuNRs formulations. Pluronic-encapsulated AuNRs showed remarkably low cytotoxicity thus indicating their potential for in vitro imaging applications. Dark-field imaging and local hyper-spectra analysis confirm the uptake of Pluronic-encapsulated AuNRs by the cancer cells. We believe that the prepared Pluronic-encapsulated AuNRs model herein can open many new opportunities towards the development of real-time in vivo AuNRs probes for photothermal imaging and photodynamic therapy. 


\section{Chapter 5 Bioconjugated AuNSs and AuNRs as Label-free Plasmon Scattering Probes for Ultrasensitive Multiplex Dark-field Imaging of Cancer Cells}

\subsection{Introduction}

AuNPs have been intensively investigated and used as contrast agents for bio-imaging because they do not photobleach and have strong elastic light scattering properties, thus bringing their detection limit to as low as $10-16 \mathrm{M}$ [107]. Typically, the SPR range of AuNSs can be tuned from 520 to $610 \mathrm{~nm}$ [108] while AuNRs have longitudinal SPR that can be manipulated ranging from 620 to $1300 \mathrm{~nm}$ by carefully modifying the reaction parameters during the synthesis process [18]. These advantages make AuNSs and AuNRs promising candidates for ultrasensitive bio-imaging applications.

To date, only a handful of manuscripts are available in the literature reporting the use of AuNPs as plasmon scattering probes for multiplex dark-field imaging of live cancer cells. Quantum dots and semiconductor nanowires are commonly used for fluorescence multiplex imaging of cells [109-111]. However, toxicity concerns from these nanoparticles formulation often limit their applications for imaging and sensing in vivo. The use of AuNPs for SERS imaging is another alternative method for multiplex imaging of cells. Here, the AuNPs are tagged with strongly active Raman molecules (Raman 
reporters), which can be conjugated with biomolecules to render specific targeted imaging both in vitro and in vivo $[112,113]$. Such nanoconstructs are termed as SERS nanotags or SERS dots in analogy with quantum dots in fluorescence. In general, the sensitivity of SERS nanotags depends on the signal intensity generated by reporter molecules. However, the limited availability of sensitive Raman reporter molecules that can be excited at NIR wavelengths poses challenge in applying it for in vivo imaging applications. Moreover, these reporter molecules are often not biocompatible which poses another obstacle for imaging application. To avoid these limitations, it is always preferable to develop label-free multiplex imaging probes for high contrast imaging applications. In this work, we report the use of bioconjugated AuNSs and AuNRs as plasmon scattering probes for imaging of live cancer cells. The AuNSs and AuNRs can be easily differentiated using spectral imaging analyzer incorporated within dark-field microscopy, for simultaneous detection of bioconjugated AuNSs and AuNRs labeled cancer cells. More specifically, for this experiment we have prepared AuNSs with an SPR peak at $537 \mathrm{~nm}$ and two types of AuNRs formulations with longitudinal SPR peaks at $625 \mathrm{~nm}$ and $747 \mathrm{~nm}$, respectively. These AuNSs and AuNRs formulations were characterized by UV-vis absorption spectra analysis and TEM. AuNSs and AuNRs surfaces were functionalized with modified poly(ethylene glycol) (PEG), and their colloidal stability was studied by using DLS and their cytotoxicity was evaluated using CCK assay. They were then conjugated with 
monoclonal anti-epidermal growth factor receptor (anti-EGFR) antibody to target EGFR-overexpressing OSCC cells. OSCC cells were incubated with the three types of bioconjugated AuNSs and AuNRs and subsequently the treated cells were imaged using dark-field microscopy. The scattering spectra of the AuNSs and AuNRs were determined by hyper-spectral analysis integrated within the dark-field imaging system. We demonstrated that AuNSs and AuNRs can be used as distinctive plasmon scattering probes for multiplex imaging of cancer cells using dark-field imaging system.

\subsection{Experimental}

\subsubsection{Materials}

Hydrogen tetrachloroaurate (III) trihydrate $\left(\mathrm{HAuCl}_{4} \bullet 3 \mathrm{H}_{2} \mathrm{O}\right)$, cetylmethylammonium bromide (CTAB), sodium borohydride $\left(\mathrm{NH}_{4}\right)$, silver nitrate $\left(\mathrm{AgNO}_{3}\right)$, L-ascorbic acid, Trisodium citrate $\left(\mathrm{Na}_{3} \mathrm{C}_{6} \mathrm{H}_{5} \mathrm{O}_{7}\right)$, Ethyl(dimethylaminopropyl) carbodiimide (EDC), N-Hydroxysuccinimide (NHS) and cell counting kit (CCK8) were purchased from Sigma-Aldrich. HS-PEG-COOH (HS- $\left.\mathrm{C}_{2} \mathrm{H}_{4}-\mathrm{CONH}-\mathrm{PEG}-\mathrm{C}_{3} \mathrm{H}_{6}-\mathrm{COOH}\right)$ and PEG-SH $\left(\mathrm{CH}_{3} \mathrm{O}-\mathrm{PEG}-\mathrm{SH}\right)$ were purchased from Rapp Polymere. Mouse monoclonal IgG1 (anti-EGFR) antibody were purchased from Santa Cruiz Biotechnology. Dulbecco's modified Eagles's medium (DMEM) and 1x phosphate buffer sulphate (PBS) were prepared in-house. Fetal bovine serum (FBS) and penicillin/streptomycin (Pen Strep) were purchased from Gibco®. 
Clean-mount solution to fix glass cover slip over 8-chamber slides was purchased from Electron Microscopy Sciences.

\subsubsection{AuNSs Synthesis}

AuNSs is synthesized by the Turkevich method [13]. $19 \mathrm{~mL}$ of $0.526 \mathrm{mM}$ $\mathrm{HAuCl}_{4}$ was boiled while stirring. $1 \mathrm{~mL}$ of $30 \mathrm{mM} \mathrm{Na} \mathrm{C}_{6} \mathrm{H}_{5} \mathrm{O}_{7}$ was then added to the solution. Boiling was continued until 10 minutes after the solution turned wine red. The solution was cooled to room temperature and stored at $4^{\circ} \mathrm{C}$.

\subsubsection{PEG functionalization of AuNRs and AuNSs}

$1 \mathrm{ml}$ of $10 \mu \mathrm{M}$ of HS-PEG-COOH was added to $1 \mathrm{ml}$ of synthesized AuNRs and AuNSs solution respectively. After 30 minutes mixing, $10 \mu \mathrm{l}$ of $0.5 \mathrm{mM}$ PEG-SH was added to solution and was left to mix for 2 hours for full coverage. The solutions were then centrifuged to remove excess HS-PEG-COOH and PEG-SH and resuspended in HPLC water. Concentrations of the solutions were fixed at optical density 1.5 with UV-vis spectrophotometer.

\subsubsection{Anti-EGFR Antibody Conjugation of AuNRs and AuNSs}

$1 \mu \mathrm{L}$ of $25 \mathrm{mM}$ EDC and $1 \mu \mathrm{L}$ of $25 \mathrm{mM}$ NHS were added to the PEG functionalized AuNRs and AuNSs solutions to activate the carboxylic acid functional groups. After 10 minutes of mixing, $50 \mu \mathrm{L}$ of $200 \mu \mathrm{g} / \mathrm{mL}$ anti-EGFR antibody was added to the solution and incubated at $25^{\circ} \mathrm{C}$ for 4 hours and then was kept at $4{ }^{\circ} \mathrm{C}$ for overnight. The solution was then washed 
by centrifugation to remove excess anti-EGFR antibody and other intermediate products, and was resuspended in $0.1 \mathrm{x}$ PBS solution. The bioconjugated AuNRs and AuNSs solution were kept at $4{ }^{\circ} \mathrm{C}$ for storage. Concentrations of the solutions were fixed at optical density 1.5 with UV-vis spectrophotometer.

\subsubsection{Dynamic Light Scattering and $\zeta$-potential}

The hydrodynamic particle size and surface $\zeta$-potential of the AuNPs were measured using a zetasizer (Malvern Nano ZS). Briefly, samples were diluted in solvent of choice and injected into a Malvern capillary cell and placed in the zetasizer. The hydrodynamic radius of the particle was then measured using DLS. The same sample was subjected to Laser Doppler Micro-electrophoresis which measures the velocity of the particles across the electrodes and enables the calculation of the zeta potential.

\subsubsection{Other Experiments}

The experimental detail of AuNPs seed synthesis, AuNRs synthesis, TEM, UV-vis absorption, cell culture and cell viability, sample preparation for dark-field microscopy and dark-field imaging are described in the Chapter 3.

\subsection{Results and Discussion}

\subsubsection{PEGylation of AuNRs and AuNSs}

In this study, two types of AuNRs were synthesized, with longitudinal SPR peaks at 626 and $754 \mathrm{~nm}$, respectively. AuNSs with SPR at $537 \mathrm{~nm}$ was also 
prepared. From the absorption spectra in Figure 5.1(a), it can be observed that the SPR spectra of AuNSs and AuNRs are distinctively different with peaks separation of $\sim 100 \mathrm{~nm}$. The TEM images of AuNSs and AuNRs are shown in Figure 5.1(b) (c) and (d). The AuNSs have diameter of $\sim 60 \mathrm{~nm}$ and the aspect ratios for the AuNRs are 2.5 and $\sim 4.5$, respectively.

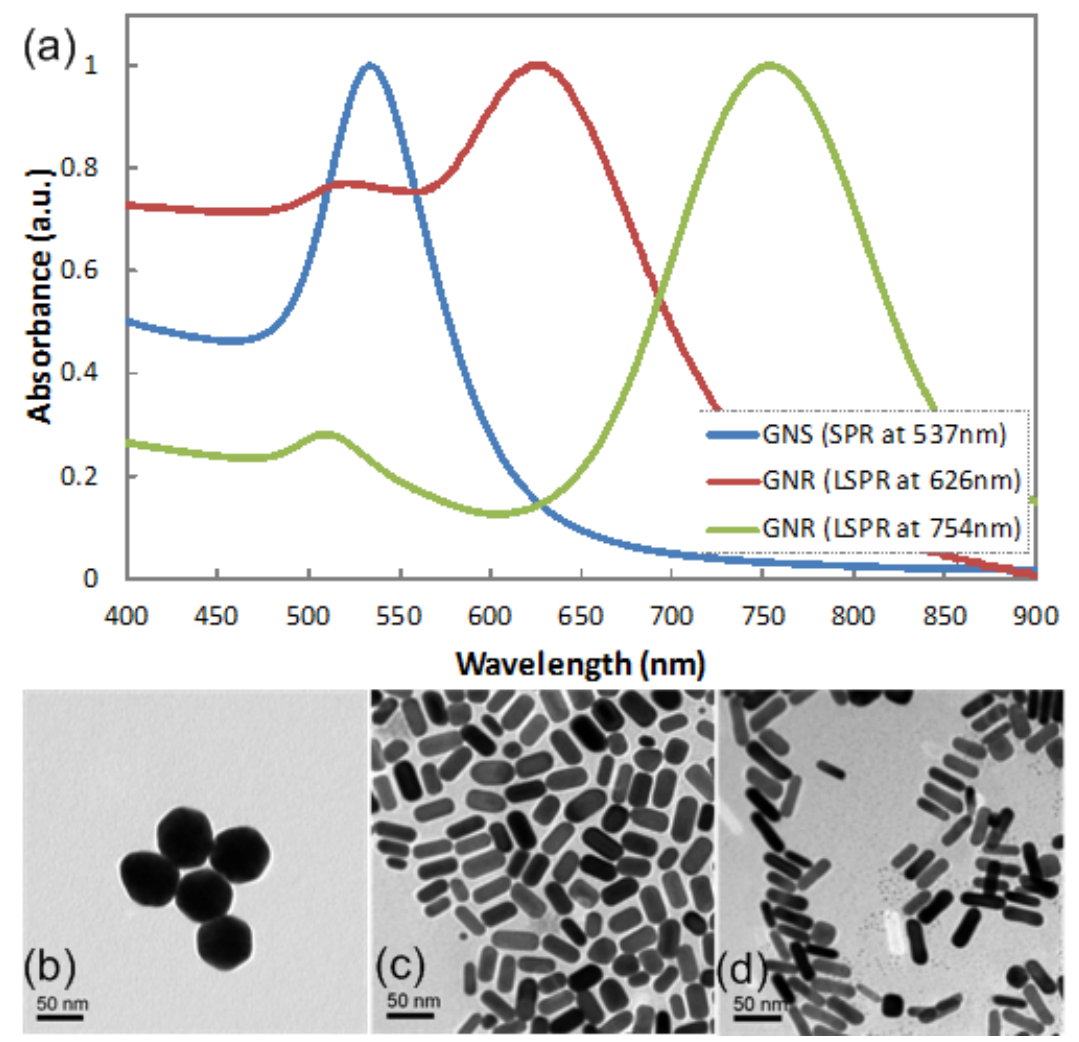

Figure 5.1 (a) The normalized UV-vis spectra and TEM images of (b) AuNSs (GNS) with SPR at $537 \mathrm{~nm}$ (c) AuNRs (GNR) with longitudinal SPR at 626 $\mathrm{nm}$ and (d) AuNRs (GNR) with longitudinal SPR at $754 \mathrm{~nm}$. (scale bar=50 $\mathrm{nm})$

During AuNRs synthesis, the CTAB forms a tightly bound cationic bilayer on the AuNR surface with the cationic trimethylammonium head group exposed 
to the aqueous media, which is suggested to pose in vitro and in vivo cytotoxicity. Washing off excess unbounded CTAB in AuNRs solution by centrifugation can slightly reduce the cytotoxicity. Nonetheless, a large amount of CTAB bilayer still remains on the surface of AuNRs and further removal of CTAB by centrifugation will result in aggregation of AuNRs. To overcome this challenge, one needs to passivate the AuNRs surface with biocompatible ligands. The functionalization of AuNRs with PEG-SH has been widely reported and PEGylated nanoparticles are known to biocompatible and can be used for in vitro and in vivo applications without observing any harmful effects [84]. In this work, the CTAB bilayers on AuNRs surface were replaced with combination of both HS-PEG-COOH and PEG-SH molecules. It was reported that surface CTAB displacement occurs owing to chemisorption of the thiol moiety of PEG (the binding energy of $\mathrm{Au}-\mathrm{S}$ is approximately $-44 \mathrm{kcal} / \mathrm{mol}$ ) [88]. Briefly, as shown in Figure 5.2, HS-PEG-COOH solution was mixed with AuNRs solution for functionalization. PEG-SH solution was then added for further encapsulation of AuNRs. Same protocol has been adopted to prepare PEGylated AuNSs. Figure 5.3 shows the $\zeta$-potential of AuNSs and AuNRs before and after PEGylation. Dramatic changes between the cationic CTAB bonded AuNRs and anionic PEG functionalized AuNRs can be observed while citrate-stabilized AuNSs show negligible changes in $\zeta$-potential after PEGylation. 


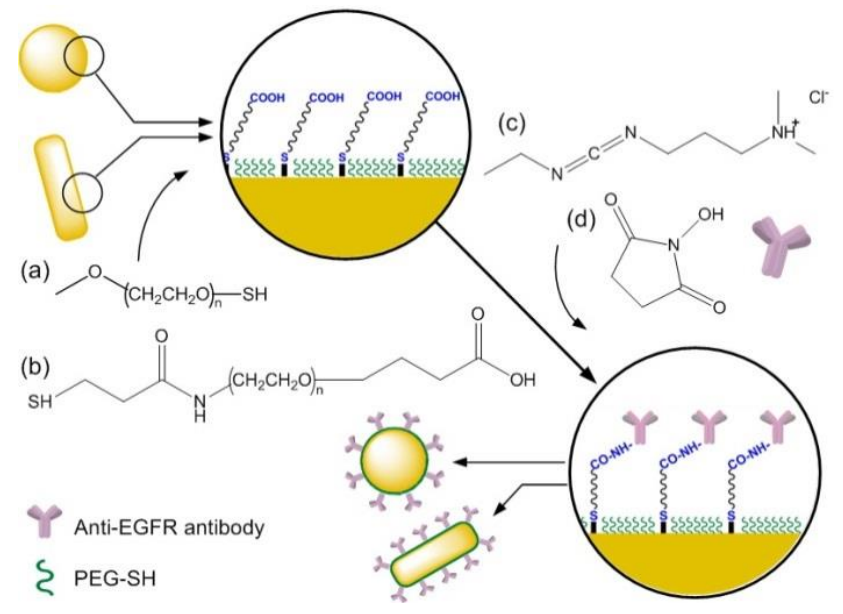

Figure 5.2 AuNPs (AuNRs and AuNSs) functionalization process by using:

(a) PEG-SH and (b) HS-PEG-COOH, and bioconjugation process by using (c) EDC, (d) NHS and anti-EGFR antibody.

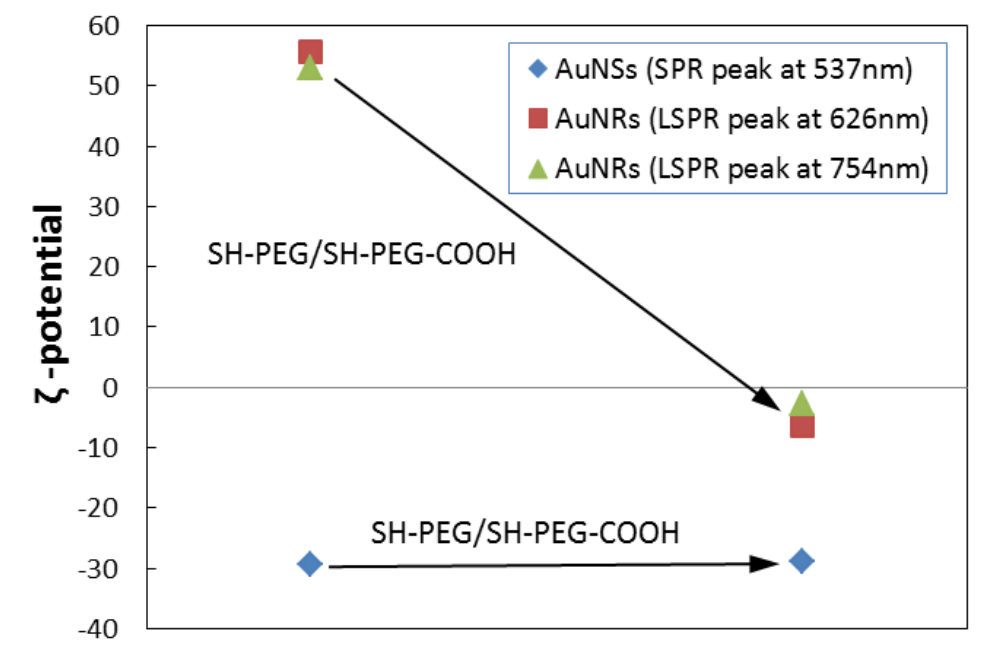

Figure $5.3 \zeta$-potential of AuNSs and AuNRs before and after PEGylation.

\subsubsection{Colloidal Stability of PEGylated AuNSs and AuNRs}

To study the colloidal stability of the aqueous dispersion of PEGylated AuNSs and AuNRs, a DLS study was performed to determine the hydrodynamic diameter of these nanoparticle formulations. The time dependent profile of the effective hydrodynamic diameter of the PEGylated AuNRs formulations and 
PEGylated AuNSs formulation is shown in Figure 5.4. Over the time range from 1 to $800 \mathrm{~min}$, the variation of their effective diameters was observed to be less than 10\%, thus indicating that the PEGylated nanoparticle formulations are colloidally stable in PBS buffer. It is worth noting that DLS can only provide an average spherical hydrated diameter, thus the effective diameters of AuNRs provided by DLS do not accurately represent their actual dimensions. However, the results are sufficient to guide us to understand their colloidal stability.

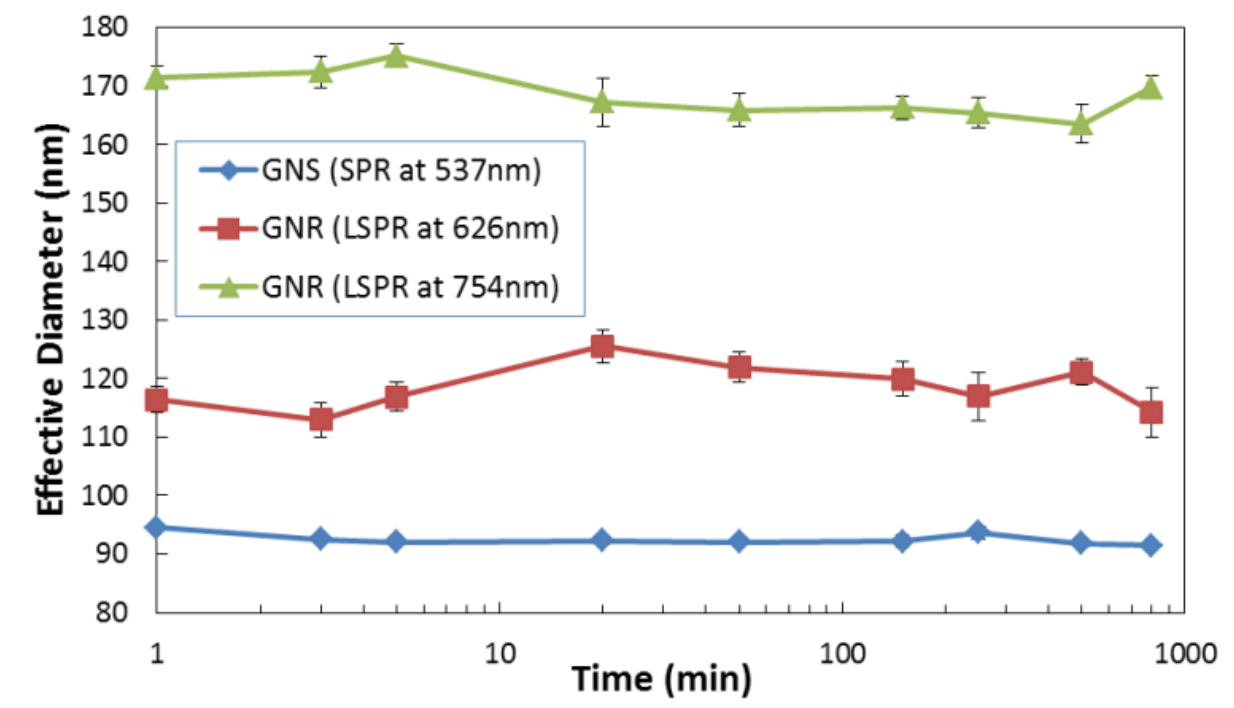

Figure 5.4 Time-denpendent profile of the effective diameter of the PEGylated AuNSs (GNS) and AuNRs (GNR) in PBS buffer solution.

\subsubsection{Cell viability of PEGylated AuNSs and AuNRs}

Cell viability studies were performed to evaluate the cytotoxicity of the PEGylated AuNSs and AuNRs with OSCC cells using CCK assay. In this experiment, the CTAB-coated AuNRs were used as control and they were 
compared to PEGylated nanoparticle formulation. The concentration for AuNSs and AuNRs solutions were fixed at optical density of 1.5. Cell viability result showed that high cytotoxicity (32.5\% viability) was observed for CTAB-coated AuNRs (see Figure 5.5). On the contrary, AuNSs synthesized with trisodium citrate are non-toxic to the cells due to the biocompatible citrate stabilization. After PEG functionalization, both AuNRs and AuNSs formulations maintained more than $98 \%$ cell viability, demonstrating their excellent biocompatibility for biomedical applications. This suggests that PEGylated AuNSs and AuNRs can be safely used as efficient plasmon scattering probes for bio-imaging applications at non-cytotoxic dosages.

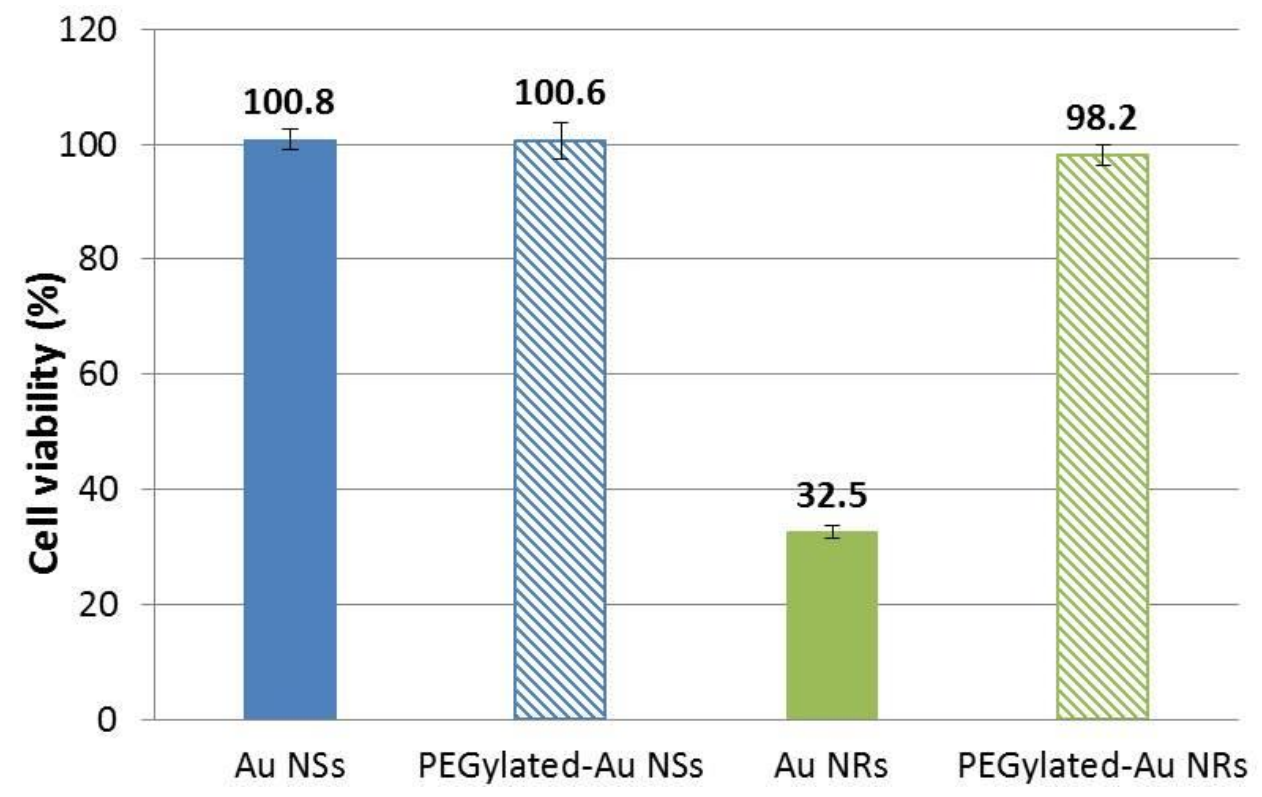

Figure 5.5 Cell viability of bare AuNSs, bare AuNRs (after washing), PEGylated AuNSs and PEGylated AuNRs 


\subsubsection{Conjugated AuNRs and AuNSs for Dark-field Multiplex Imaging of Cancer Cells and Its Potential Applications}
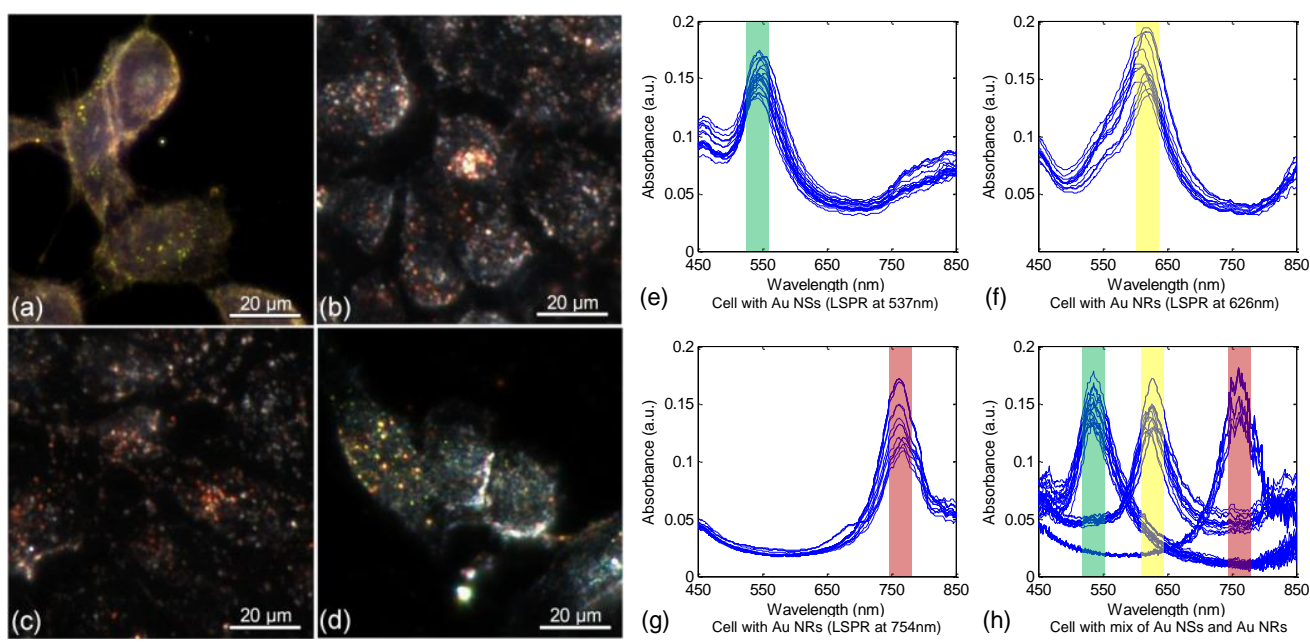

Figure 5.6 Dark-field images of OSCC cell line incubated with (a) AuNSs

with SPR peak at $537 \mathrm{~nm}$, (b) AuNRs with longitudinal SPR peak at $626 \mathrm{~nm}$,

(c) AuNRs with longitudinal SPR peak at $754 \mathrm{~nm}$ and (d) a mixture of three types of AuNPs. The hyper-spectra taken from dark-field from (a), (b), (c) and (d) were shown in (e), (f), (g) and (h) respectively.

The PEGylated AuNRs and AuNSs were conjugated with antibody for dark-field multiplex imaging of cancer cells. As shown in Figure 5.2, EDC and NHS were added to the PEGylated nanoparticles solution to activate the carboxylic acid function groups. Anti-EGFR antibody was then added to the solution and they were covalently linked to the PEG surface. The bio-conjugated AuNSs and AuNRs formulations were then treated with OSCC cells. After two hours of incubation, the cells were washed and subsequently imaged under a dark-field microscopy. Hyper-spectral imaging analysis was used to collect the scattering spectra of every pixel in an image map under the 
dark-field microscopy and this will allow us to identify the cells labeled with bioconjugated AuNSs and AuNRs. More importantly, the imaging analysis would capture unique scattering spectra of the AuNSs and AuNRs while the cells that are not labeled with AuNPs would show a baseline spectrum. Since scattering caused by cell is much weaker than that of AuNPs, and given the distinct frequencies of scattering from the different AuNPs, the hyper-spectral analysis allows the discrimination of each nanoparticle type (AuNSs or AuNRs). For the in vitro multiplex imaging study, the OSCC cells were incubated with the AuNSs and AuNRs formulations individually, and another set of OSCC cells were incubated with the mixture of AuNSs and AuNRs formulation. The dark-field images and hyper-spectra of these cells are shown in Figure 5.6. In Figure 5.6(a), the uptake of AuNSs in the cells were evident from the presence of green scattering color, while red scattering color was observed from AuNRs, as shown in Figure 5.6(b) and (c). The green and red scattering color spots in Figure 5.6(d) clearly indicated the robust receptor-mediated cellular uptake of both AuNSs and AuNRs bio-conjugates. The hyper-spectra (Figure 5.6(e), (f), (g) and (h)) taken from green/red scattering color spots confirmed that these color spots originated from AuNSs and AuNRs. The hyper-spectra obtained from the samples match well to their UV-vis absorbance spectra in Figure 5.1(a). It is worth noting that AuNPs aggregation was commonly observed in biological environment, and it may results spectral shift or broadening. However, the clear differentiated spectra 
in Figure 5.6 indicate that PEG functionalized AuNPs are stable in biological environment. Control experiments were also performed to verify that bioconjugates are specifically taken up through the receptor-mediated interaction. In the control experiment, the SKOV3 cells were incubated with anti-EGFR conjugated AuNRs and they were served as negative control. Figure 5.7(a) shows the OSCC cells in the absence of both bio-conjugated AuNSs and AuNRs. No color scattering spots were observed in the dark-field images in comparison to the images in Figure 5.6. As shown in Figure 5.7(b), since the SKOV3 cells line does not overexpress EGFR receptors, no uptake of bio-conjugates was observed, confirming the specific receptor-mediated nature of the uptake of the bio-conjugated AuNSs and AuNRs.
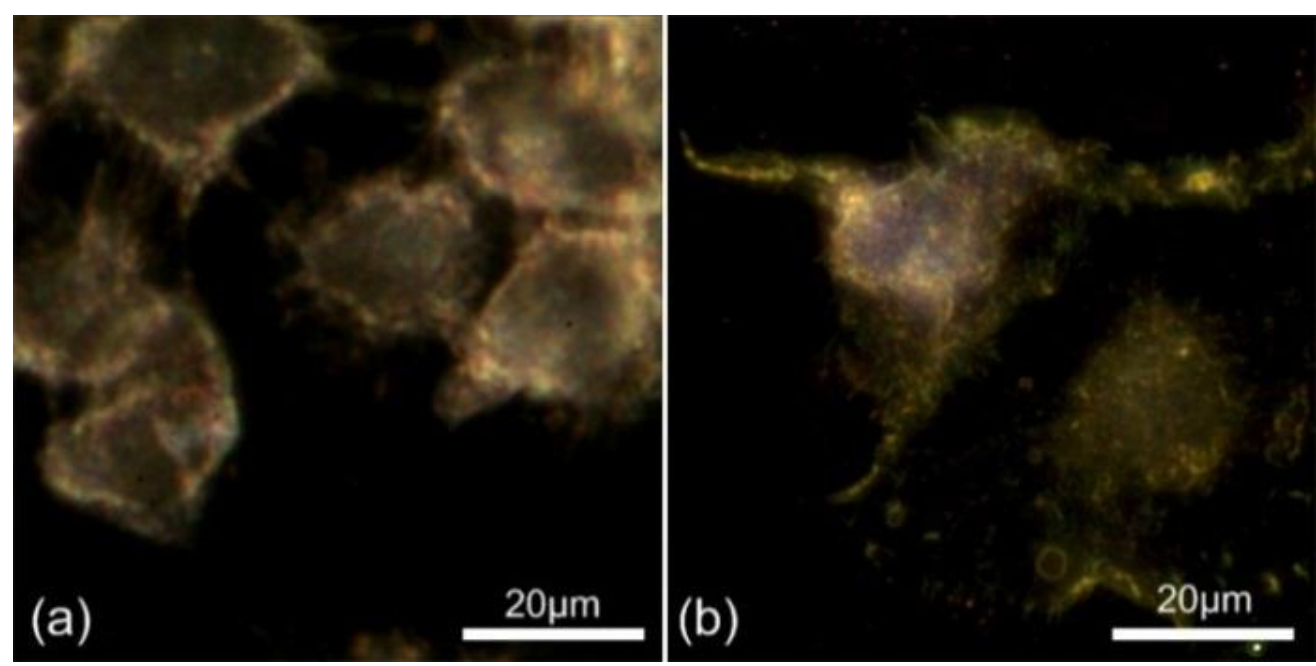

Figure 5.7 Dark-field images of (a) bare OSCC cell line and (b) SKOV3 cell line incubated with anti-EGFR antibody conjugated AuNRs.

The experimental results supported our concept of using AuNSs and AuNRs as plasmon scattering probes for multiplex dark-field imaging. In comparison 
to previously reported multiplex imaging methods, our approach is facile and straightforward. More importantly, the optical property of AuNSs and AuNRs probes can be systematically tuned to provide higher spatial resolution over a wide wavelength range for dark-field imaging. Our experiment shows that with SPR absorption peak interval of $\sim 100 \mathrm{~nm}$, up to 7 plasmon scattering color probes can be used, with their SPR absorption peaks ranging from 520 to $1300 \mathrm{~nm}$. This unique feature will allow one to study the interaction of several cancer biomarkers with cancer cells at the same time where one can investigate the specificity uptake of individual markers with in the cancer cells.

This study shows that AuNPs can be served as multiplex plasmon scattering probes for dark-field imaging. More importantly, we envision that the developed multiplex nanoimaging platform can be used for drug and gene delivery studies. AuNPs can be conjugated with antibiotics or other drug molecules via ionic or covalent bonding, or by physical absorption. For example, AuNSs were conjugated with methotrexate, an analogue of folic acid that can destroy folate metabolism of cells, for cancer therapy [114]. Antibiotics such as ampicillin, streptomycin, and kanamycin can also be conjugated to AuNPs to provide a greater degree of inhibition of the growth of bacteria than the free forms of the antibiotics [115]. Many surface modification methods of AuNPs have been proposed for drug delivery. Besides the widely adopted PEG coating modification, folic acid can also be 
conjugated to AuNPs playing an important role for folate receptor-targeted drug delivery or targeted therapy [116]. 3-mercapto-propionic acid (MPA) and $\mathrm{NH}_{2}$-PEG-NH $\mathrm{N}_{2}$ were recently adopted to functionalize AuNPs and target payload to the cell nucleus [117]. The AuNPs based drug delivery platform can improve the bio-distribution, stability, and pharmacokinetics of drugs. They can also carry nuclei acid, used in gene delivery, to treat diseases. It has been reported that functionalizing AuNPs with cationic quaternary ammonium groups and then binding plasmid acid on them can be used to protect the DNA from enzymatic degradation and could regulate DNA transcription of T7 RNA polymerase [118]. Recently, it was reported that AuNRs can be used as a capsule for controlled drug release application. The AuNRs with different aspect ratio were separately conjugating two different DNAs, namely FAM-DNA and TMR-DNA, respectively. After irradiation by a laser with a wavelength of $800 \mathrm{~nm}$, shorter AuNRs melted and $70 \%$ of attached FAM-DNA released while only $10 \%$ of the TMR-DNA that attached on long AuNRs was released [119]. This AuNRs based selective release system, together with all the preliminary drug and gene delivery studies mentioned above, indicates that our developed AuNPs based multiplex dark-field imaging platform can be easily integrated into the studies mentioned above for more in-depth detail studies, and may provide real-time monitoring of the interaction between AuNPs and biological environment. 


\subsection{Summary}

In conclusion, we demonstrated the use of AuNSs and AuNRs as label-free plasmon scattering probes for multiplex dark-field imaging of cancer cells. The unique absorption spectra from AuNSs and AuNRs can be easily discriminated by using hyper-spectra analysis system integrated with a dark-field microscopy imaging system. In comparison to previously reported multiplex optical probes, AuNPs can be prepared through a facile and simple synthesis method and they can be translated to clinical applications due to their low toxicity. Due to the narrow emission wavelength range (from 500 to $800 \mathrm{~nm}$ ) of the light source, we adopted one type of AuNSs and two types of AuNRs with SPRs absorption peaks located in that range. As a result, we successfully demonstrated the concept of AuNPs-based plasmon scattering multiplexing probes. Based a broadband white light excitation source, and given the tunable longitudinal SPR range of AuNPs from 620 to $1300 \mathrm{~nm}$, it can be estimated that up to 7 multiplexing probes with $100 \mathrm{~nm}$ SPR absorption wavelength interval can be utilized for molecular distinction. 


\section{Chapter 6 Optimized Sandwiched Surface Plasmon Resonance Enhanced Bio-sensor for Multiplex Biomarker Detection}

\subsection{Introduction}

Surface plasmon resonance (SPR) based sensors have been widely used to study biomolecule interaction during recent years. Generally, when a light beam is totally reflected from the interface between waveguide and outer medium, an evanescent filed will propagate along the surface of interface. This principle is most implemented by the widely used Kretschmann configuration [120], when a metal (typically gold for its chemical stability) film is coated on the surface of glass prism. SPR will be stimulated when the frequency of photons matches the natural frequency of surface electrons oscillating against the restoring force of positive nuclei, which can be detected by measuring the absorption of reflected light. SPR is highly sensitive to the external medium, and even a small refractive index change at the metal film/analyte surface will result in a significant shift of wavelength or light incidence angle for SPR [121]. SPR bio-sensors use this phenomenon to measure the amount of bound analyte and subsequently the concentration of analyte in a sample. Thus, the changes in the refractive index due to analyte binding to the specific receptor, which is immobilized on a gold film coated sensing surface can be monitored by SPR bio-sensor [122]. 
There is an increasing demand of developing bio-sensor devices which are capable of detecting biomarker proteins for human diseases. These applications require bio-sensors technologies to be sensitive, rapid and cost-effective. SPR bio-sensor technology is a well-established technique and has advantages such as real-time monitoring of molecular interactions [123], specific for a variety of different analytes [124] and comparably low cost and high sensitivity [125]. Many types of detection formats have been developed for SPR sensors based on type of targeting analytes, binding characteristics and concentration of samples. The most commonly adopted formats as described by J. Homola are direct binding format, sandwiched binding format, competitive binding format and inhibition binding format. Giving that the typical small amount of analytes (e.g. protein with concentration less than $\mathrm{nM}$ ) presented in a sample to be measured by SPR usually cannot generate a sufficient refractive change (e.g. $10^{-5}-10^{-8}$ RIU) [126], sandwiched binding format receives more attention due to its improvement on sensitivity.

When combining metallic nanoparticles with SPR bio-sensors as a sandwiched structure, the metallic nanoparticles not only induce the increase in binding mass and refractive index, but also combination of LSPR with SPR, which perturbs the evanescent field thus led to enhanced sensitivity. Natan et al. demonstrated the use of AuNPs in SPR sensors to increase the sensitivity through the binding of a secondary antibody in the sandwich detection format in 1990s [127, 128]. An SPR based AuNSs labeled sensitive kinetic assay for 
carbamate-acetylcholinesterase interaction has been developed by Zhang's group [129]. Recently, a sensitivity improved SPR bio-sensor for biomarker detection has been described by Prasad et al. [130]. Instead of using AuNSs, they adopted AuNRs for their unique behavior on tunable longitudinal plasmonic peak which enables an effective plasmonic coupling between the sensing film and the nanoparticle. By applying this platform they detected tumor necrosis factor alpha (TNF- $\alpha$ ) antigen with extreme high sensitivity (estimated to be $0.03 \mathrm{pM}$ ), which is $1-2$ orders of magnitude higher than the traditional immunoassay method. Inspired by this method, in this work, we systematically studied the effect of AuNRs dimensions, AuNRs-to-film distance and gold film thickness on SPR sensor. We then propose an optimized sandwiched SPR bio-sensor for multiplex biomarker detection by using gold sensing film coupling with AuNRs with different aspect ratios.

\subsection{Simulation Model}

\subsubsection{Model Setup}

As shown in Figure 6.1(a), the glass block used in the sandwiched SPR biosensor setup is evenly coated with a thin gold layer that plays the role as a sensing film. In order to introduce the AuNRs forming the sandwiched structure for biomarker detection, the sensing film surface is first immobilized with capture antibodies. Then the sample solution containing biomarkers, which are highly specific to the capture antibodies, are be applied to the surface. After the biomarkers are captured by antibodies on the surface, a 
solution of AuNRs functionalized with capture antibodies will be applied to the film surface. They will bind to the captured biomarkers on the sensing film surface thus to form the second layer for the sandwiched structure, as shown in Figure 6.1(b). According to Prasad's experimental result, the AuNRs are able to vertically bind to the film surface [130]. When light is projected onto the interface between the glass block and gold film at a certain incident angle, the SPR and longitudinal SPR will occur and the reflected light will have an absorption peak at a specific wavelength, the biomarkers can be detected. Since SPR and longitudinal SPR are highly sensitive to environment changes, we need to carefully study the effect of AuNR dimensions, AuNR-to-film distance and gold film thickness on SPR and optimize the system for multiplex biomarker detection.

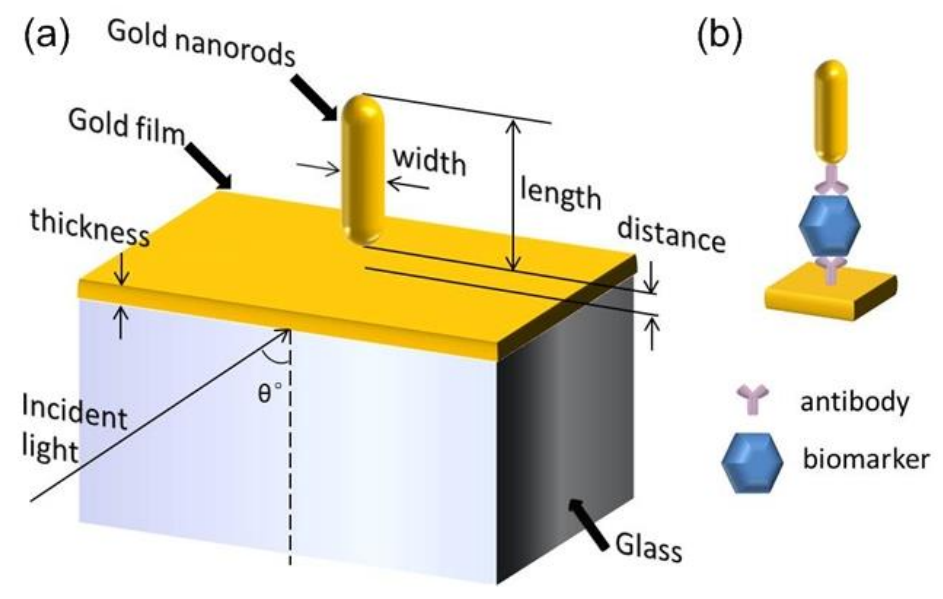

Figure 6.1 (a) Schematic of a sandwitched SPR-enhanced bio-sensor model.(b) Sandwichted structure formed by AuNR, biomarker and gold sensing film. 


\subsubsection{Simulation Setup}

The studies were performed by using FEM simulation software COMSOL Multiphysics. The model was solved in 2D RF module, where TM in-plane waves were adopted in order to excite the SPR. The TM planar waves (scanning from $0.7 \mu \mathrm{m}$ to $1.2 \mu \mathrm{m}$ ) were generated at a port boundary condition from the bottom of the glass propagates along y direction which is defined as

$$
\begin{aligned}
& k 1 x=k 0 \_r f w h \times N \times \cos (\alpha) \\
& k 1 y=k 0 \_r f w h \times N \times \sin (\alpha) \\
& H 0 z=\exp (-i \times k 1 y \times x)
\end{aligned}
$$

where $\alpha$ is the propagation angle, $k 0 \_r f w h$ is a built-in parameter for free space wave number. $N=(n-i k)$, which refers to the refractive index of medium, $k 1 x$ and $k 1 y$ are the propagation constants. Along the respective axes, since the angle of incident is introduced, we use the Floquet periodic boundary condition for the left and right boundaries along the y direction. For the medium (glass, gold film and water) which light propagates in, the Floquet boundary condition is Eq. (1) and Eq. (2), $N$ in these two equations should be changed by corresponded medium refractive index. Since light will be refracted when propagates through each interface, which can be calculated through Snell's law, $\alpha$ in these two equations should also be replaced by the refraction angle in each medium respectively. The boundary condition ensures 
that a wave, when reaching the bottom boundary (glass) transmits to the top boundary (air) with the appropriate phase shift. We use perfect match layer (PML) to ensure no reflection from top boundary for calculation accuracy. The simulation is performed by using wavelength modulation through wavelength scan and Ey (y component of electrical field) will be calculated out.

\subsection{Results and Discussions}
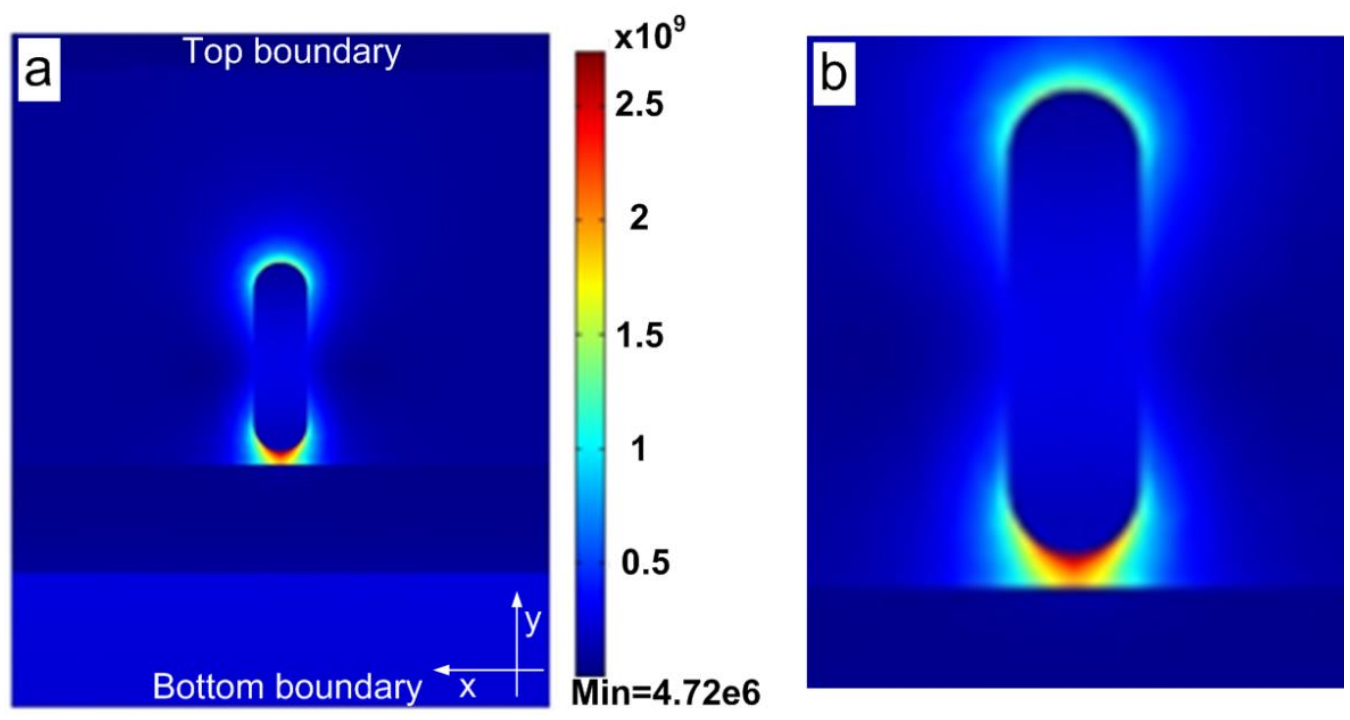

Figure 6.2 (a) Simulated electrical field distribution for AuNR over a $40 \mathrm{~nm}$ thick gold film. AuNR has a width of $20 \mathrm{~nm}$ and length of $70 \mathrm{~nm}$, and the distance between AuNR and gold film is $5 \mathrm{~nm}$. (b) Enlargement of the enhanced electrical field area in (a).

To study various parameters and their effect to SPR, the angle of incident light should be determined first. According to Homola's group's study [131, 132], when the incidence angle of the light source is set to a small value 
$\left(\alpha \leq 65^{\circ}\right)$, the SPR occurs with longer resonance wavelength and a high sensitivity. However, the SPR peak will become broader for smaller incident angles. Therefore, we fix the incident angle at $67^{\circ}$ to compromise both the sensitivity and peak width. The simulation result of electrical field distribution for AuNR over gold layer is shown in Figure 6.2 (a) and (b). A significant enhancement of electrical field (hot spot) can be observed from the hotspot between AuNR and gold layer.

\subsubsection{Gold Film Thickness}

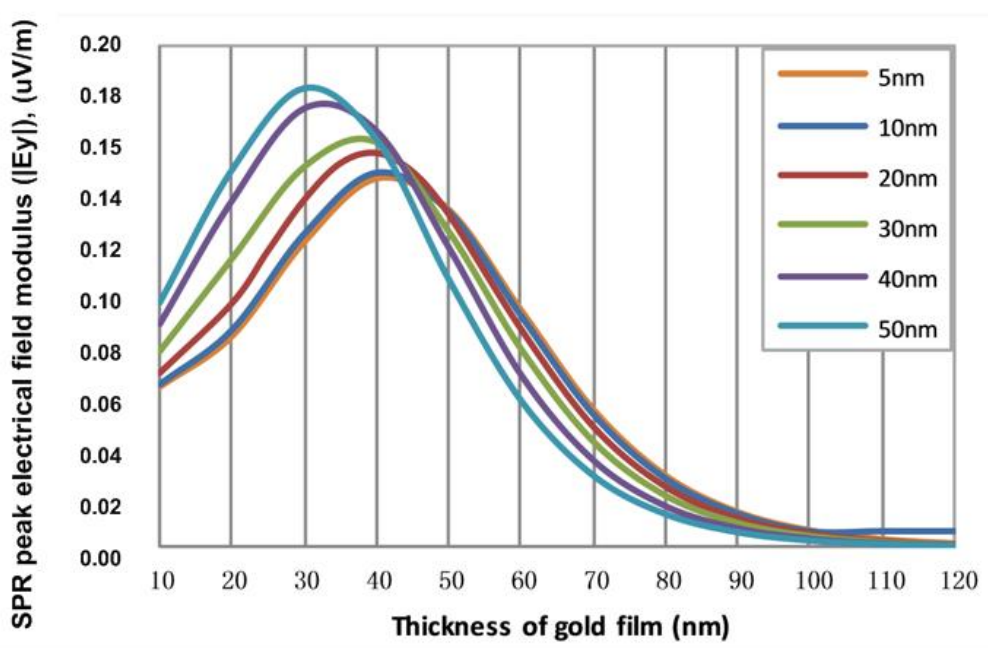

Figure 6.3 The SPR peak E-field modulus for gold film thickness varies from $10 \mathrm{~nm}$ to $120 \mathrm{~nm}$ for different AuNRs sizes.

The thickness of the gold film is a very important factor for SPR enhancement. In this study, the distance between AuNR and gold film is fixed at $10 \mathrm{~nm}$. To simplify the simulation, we treat AuNR as a cube, thus the width and length are equal. In order to find the optimal thickness which gives the maximum SPR enhancement, for each cube size, the SPR peak E-field for 
different gold film thickness is calculated by wavelength scan. As shown in Figure 6.3, when AuNR size increases from $5 \mathrm{~nm}$ to $50 \mathrm{~nm}$, the gold film thickness which brings maximum SPR decreases from $42 \mathrm{~nm}$ to $31 \mathrm{~nm}$. This result enables us to find an optimum thickness range according to the AuNR size we expect to use for the bio-sensor. Furthermore, it confirms that the coupling of AuNR on SPR sensing film will bring a perturbation to the evanescent field thus causing SPR intensity change. Since for most synthesizes and applications, the width of AuNRs range from $10 \mathrm{~nm}$ to $30 \mathrm{~nm}$ [133], we choose the gold film thickness to be $40 \mathrm{~nm}$ for best SPR enhancement.

\subsubsection{AuNR-to-film Distance}

The distance between AuNR and film depends on the linker, antibody and protein size. In this study, the AuNR size is set to be $20 \mathrm{~nm}$ (cube shape for simplify calculations) with a $40 \mathrm{~nm}$ thickness gold film. Since we are going to demonstrate bio-sensor for $\mathrm{IgG}$ proteins detection, consider the compound length of $\operatorname{IgG}$ protein, anti-IgG antibody and linkers is between $10 \mathrm{~nm}$ to 20 $\mathrm{nm}$, we therefore focus on the SPR effect with distance varies from $0 \mathrm{~nm}$ to 20 nm. Results in Figure 6.4 indicate the distance changes have slightly effect on SPR peak wavelength compare to the sensing film without AuNRs, it was observed that less than $10 \mathrm{~nm}$ on SPR peak wavelength shift for distance change from $10 \mathrm{~nm}$ to $20 \mathrm{~nm}$. This interesting result indicate that the bio-sensor is less sensitive to distance change, thus the dimension changes for 
different $\mathrm{IgG}$ proteins and antibodies will have negligible effect for the sensing accuracy. It should be noted that, compare with SPR without AuNR, existence of even a such small AuNR shows a significant perturbation to the SPR field, introduces not only SPR wavelength shift but SPR enhancement.

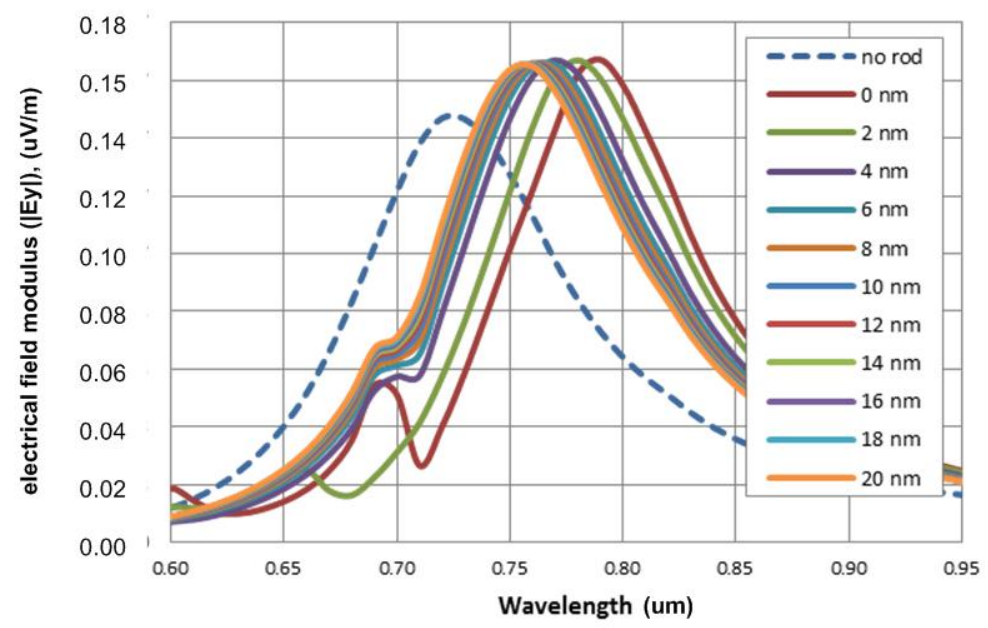

Figure 6.4 The variation of the SPR E-field modulus spectra without AuNR and with AuNR by varing AuNR-to-film distance

\subsubsection{AuNR's Length and Width}

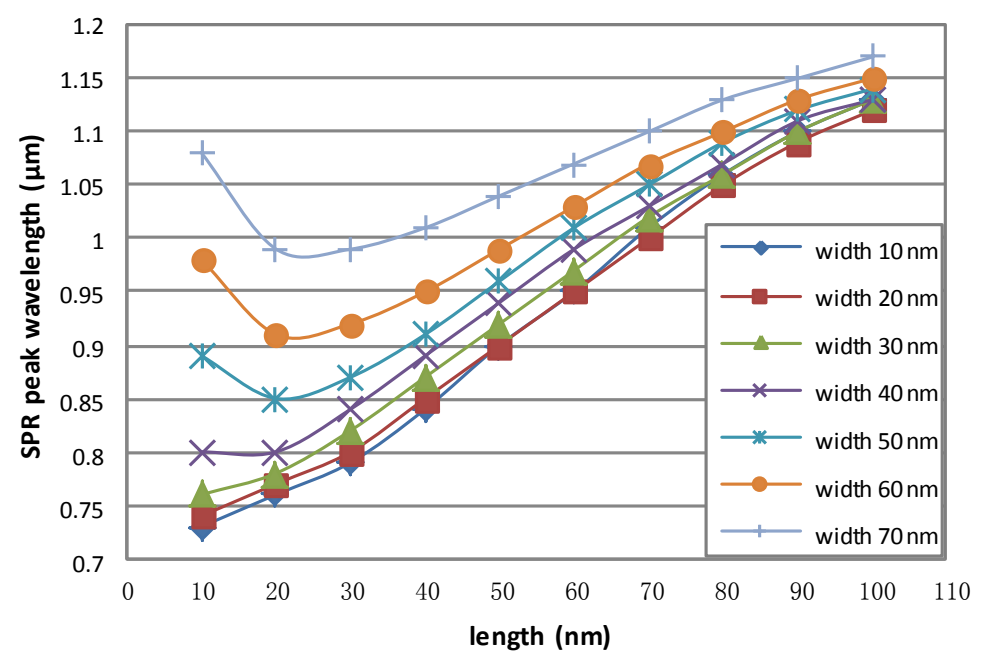

Figure 6.5 SPR peak wavelength for different AuNR width and length on gold film. 
As already observed from the above results, the existence of AuNR has strong effect onto SPR field, both the peak wavelength and intensity. It is necessary for us to study the SPR by changing width and length of the AuNR. The distance between AuNR to film is fixed at $10 \mathrm{~nm}$. Figure 6.5 shows increase either length or width results red-shift on SPR peak wavelength. However, the result indicates SPR is much more sensitive to AuNR length changes. Especially for AuNR has a length between $30 \mathrm{~nm}$ to $90 \mathrm{~nm}$ and a width between $10 \mathrm{~nm}$ to $40 \mathrm{~nm}$, SPR shifts $\sim 50 \mathrm{~nm}$ for each $10 \mathrm{~nm}$ increase on AuNR length, only less than 20nm shift for same increase on AuNR width, on the contrary. This may due to the electrical field has most components on y direction which are perpendicular to the gold film and therefore excite the longitudinal SPR mode on AuNRs. It should be noted that most type of AuNRs have dimensions in this region [18].

\subsubsection{Bio-sensor Concept for Multiplex IgG Proteins Detection}

Based on the characteristics we found, we optimized this sensing system and proposed the concept of using AuNRs with different aspect ratio for multiplex IgG proteins detection. Consider this SPR sensor is more sensitive to AuNR length changes, we select three types of AuNRs for multiplex detection. These three types of AuNRs have $20 \mathrm{~nm}$ in width and $40 \mathrm{~nm}, 60 \mathrm{~nm}, 80 \mathrm{~nm}$ in length, respectively. According to the results in Figure 6.5, these three types of AuNRs should result different SPR shift significantly. Mouse-IgG, human-IgG and rabbit-IgG proteins were chosen to demonstrate the concept. 


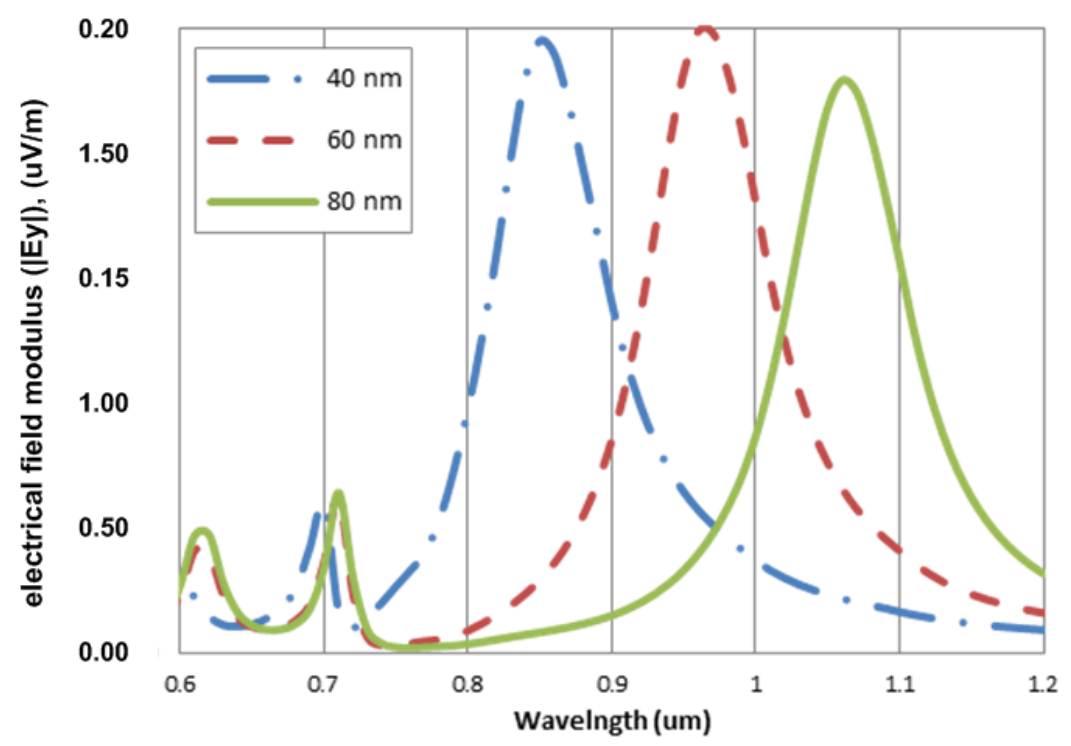

Figure 6.6 SPR absorption spectra for AuNRs with 20nm width and aspect ratio from 2 to 4 on $40 \mathrm{~nm}$ gold film with $20 \mathrm{~nm}$ distance.

Since their size different is small thus the SPR effect of distance between AuNR and film can be neglected, as we shown in Figure 6.4. Therefore, we set the AuNR-to-film distance to $20 \mathrm{~nm}$ with a $40 \mathrm{~nm}$ thick gold film. In order to perform multiplex detection, the gold film is immobilized with three types of antibodies (anti-Mouse- $\operatorname{IgG}$, anti-human- $\operatorname{IgG}$ and anti-rabbit- $\operatorname{IgG}$ ) first to specifically capture the IgG proteins. While three types of AuNRs are immobilized with these three types of antibodies correspondingly. The anti-IgG antibodies immobilization processes are well established [134]. Then sample solution contains certain type of $\operatorname{IgG}$ protein is applied onto the sensing film. At the last step, solution of AuNRs with immobilized antibodies is applied onto the film. Since only the type of AuNRs with corresponding immobilized antibody to the $\operatorname{IgG}$ protein in the sample solution will be captured to the film, therefore, the specific SPR peak which corresponding to 
the certain AuNR will exist. As shown in Figure 6.6, according to the SPR absorption peak, we are able to tell the type of AuNR and its corresponded IgG protein in the sample solution with SPR wavelength separation more than100 $\mathrm{nm}$.

\subsection{Summary}

In this work we have demonstrated AuNR combine with gold film for a SPR-based bio-sensor. The effects of AuNR dimensions, AuNR-to-film distance and gold film thickness on SPR were studied through FEM simulation. We found (i) the optimized thickness for the sensor is $\sim 40 \mathrm{~nm}$ for maximum SPR absorption; (ii) SPR sensor is insensitive to the AuNR-to-film thickness within $10 \mathrm{~nm}-20 \mathrm{~nm}$ range, (iii) Increase in AuNR length results significant SPR red-shift while the sensor shows much less sensitive to AuNR width change. Based on these results, we demonstrated adopting three types of AuNRs with $40 \mathrm{~nm}, 60 \mathrm{~nm}$ and $80 \mathrm{~nm}$ in length and $20 \mathrm{~nm}$ in width for an optimized sandwiched SPR enhanced bio-sensor for Multiplex IgG proteins detection. According to our concept and simulation result, the bio-sensor is able to detect these proteins by SPR absorption peak corresponding to different types of AuNRs. We believe this bio-sensor can achieve rapid, cost effective multiplex biomarker detection not only for IgG proteins but wide range of biomolecular interactions. 


\section{Chapter 7 Highly Sensitive SERS Detection and Quantification of Sialic Acid on Single Cell Using Photonic-Crystal Fiber with AuNPs}

\subsection{Introduction}

Glycans are compounds that are commonly found on the exterior surface of cells and play important roles in the structural, modulation and recognition functions of living cells [135]. More specifically, glycans typically correlate with the development and progression of many cancers and the changes they undergo are known to affect malignant transformation and tumor progression [136]. Sialic acid has been used as a tumor-associated carbohydrate antigen to identify cancers [137]. It is an anionic monosaccharide found in N-glycans, O-glycans and glycosphingolipids and is an essential component of glycoproteins and glycolipids on the cellular membrane [138]. The overexpression of sialic acid on a cell's surface indicates the malignant and metastatic phenotypes for various types of cancers, whereas a decrease in sialic acid expression implicates the erythrocytes of diabetic mellitus [139]. It has also been found that sialic acid-rich glycoproteins bind selectins in human organisms. When these glycoproteins are overexpressed, metastatic cancer cells experience negative charges on their membranes, creating repulsion among them and thus allowing late-stage cancer cells to enter the blood 
stream, further spreading the cancer [140]. Therefore, accurately monitoring sialic acid expression on cell surface provides an effective way to study tumor malignancy and metastasis along with many other sialic acid-related diseases. Great efforts have been made to detect and quantify sialic acid. Commonly available detection methods include colorimetric $[141,142]$ and fluorometric $[143,144]$ detection, other reported methods include chromatography spectrometry [145, 146] and potentiometry [147]. However, each of these techniques has certain drawbacks: the sensitivity of colorimetric detection is low [148]; while fluorometric detection is generally characterized by poor discrimination of specific fluorophores [149] as well as generation of broad emission spectra [150] and photobleaching [151], both of which obscure useful information. More importantly, all of these techniques require the use of sophisticated equipment, tedious preparations and large amounts of cells (usually $10^{5}$ to $10^{9}$ cells are required [152-154]) to sufficiently detect and quantify sialic acid. Therefore, the development of a simple, reliable and highly sensitive detection and quantification for the sialic acid present on a single cell remains a significant challenge.

To analyze molecules with ultra-high sensitivity, SERS is often used [155, 156]. Conventionally, SERS detection was performed on a gold or silver nanostructured substrate, which allowed the Raman signal to be enhanced by several orders of magnitude due to the strong SPR from nanostructured surfaces [45, 157]. However, as such enhancement is only effective when 
Raman tags are located very close to the substrate surface, the effective enhancement distance of the substrate is usually less than $100 \mathrm{~nm}$ [51-53], allowing only two-dimensional (2D) enhancement. As a mammalian cell is typically $\sim 20 \mu \mathrm{m}$ in diameter, a nanostructured substrate is only able to enhance the Raman signal from the bottom of the cell that is in contact with the substrate, resulting in low detection sensitivity. Optical fibers - both of solid and hollow cores, respectively - have been used together with AuNP in SERS for molecule detection $[56,158]$. Solid core fiber has typically been adopted for use in fiber probes, in which one end of the fiber is tapered, allowing light to leak from the fiber and interact with the sample attached to or near the surface of the tip $[159,160]$. However, tapering the fiber is a complicated process and the light loss from the tapered tip tends to be large [161], limiting the detection sensitivity. Hollow core photonic crystal fibers (HC-PCF) have been considered to be more favorable because samples can be pumped into the fiber through the hollow core, where the laser light transmitted inside is able to interact with the samples through the fiber with much better confinement $[58,162]$. As the light is confined in the hollow core by the band gap effect from the photonic crystal structured fiber cladding [63], when the hollow core and air holes in the cladding are filled with liquid, the band gap shifts due to refractive index change [64]. As a result, transmitted light may not be well confined, leading to larger loss and decreased sensitivity. Therefore, liquid samples must be desiccated after being pumped 
into the fiber core before detection. This platform thus becomes unsuitable for testing biomolecules, as they require an aqueous environment.

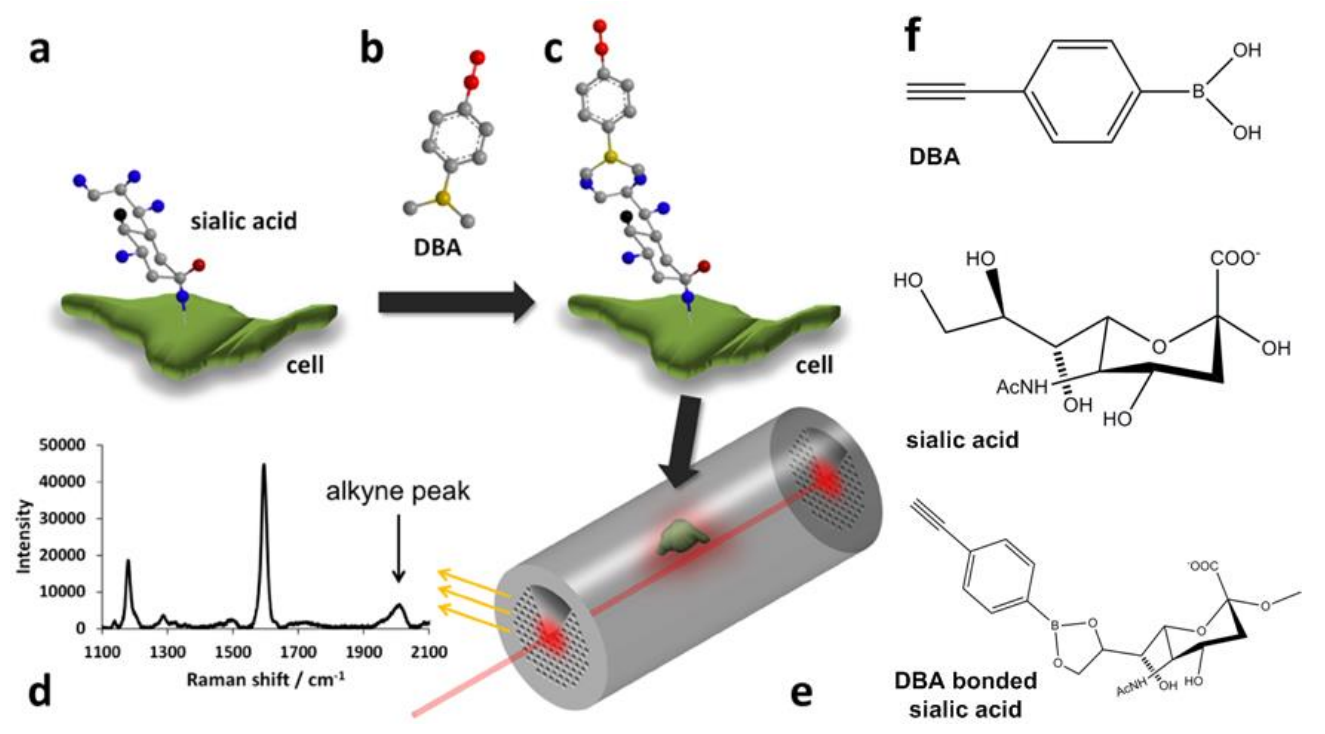

Figure 7.1 Illustration of the detection method. (a) Chemical structure of sialic acid on cell surface. (b) The chemical structure of DBA. (c) DBA-bonded sialic acid on the membrane of a cell at the C-8, 9 diol of sialic acid. (d) Raman spectrum detected from DBA tagged cell in SC-PCF, the 2,000 cm-1 peak arises from alkyne group from DBA. (e) Schematic of SC-PCF as a SERS platform for detection of sialic acid on a single cell. (f) Chemical structure of DBA, sialic acid and DBA bonded sialic acid.

Raman spectra of cell and other biomolecules lie within the region of $400 \mathrm{~cm}^{-1}$ to $1800 \mathrm{~cm}^{-1}[163,164]$. Thus, for sensitive SERS biomolecule detection, it is essential that the Raman peaks of tags fall outside of this region to avoid signal overlapping. As such, alkynes that possess a strong SERS peak around the $2,000 \mathrm{~cm}^{-1}$ area due to $\mathrm{C} \equiv \mathrm{C}$ stretching $[165,166]$ are suitable for this 
platform. Previous research reported that phenyl-boronic acid (PBA) can form favorable bonds with sialic acid among common carbohydrates on the cell surface at the physiological pH of 7.4 [167]. Recently, Liu et al. proposed the use of PBA-tagged quantum dots to label sialic acid on cell membranes for fluorescence imaging [168]. Inspired by their work, we adopt 4-(dihydroxyborophenyl) acetylene (DBA), a compound that consists of sialic acid capture moiety (phenyl-boronic) and alkyne functional group in our designed study (Figure. 7.1 (b)). By exploiting the chemistry between DBA and sialic acid (Figure. 7.1 (a), (c)), and the non-interfered alkyne Raman peak (Figure. $7.1(\mathrm{~d})$ ), sialic acid SERS detection can be realized.

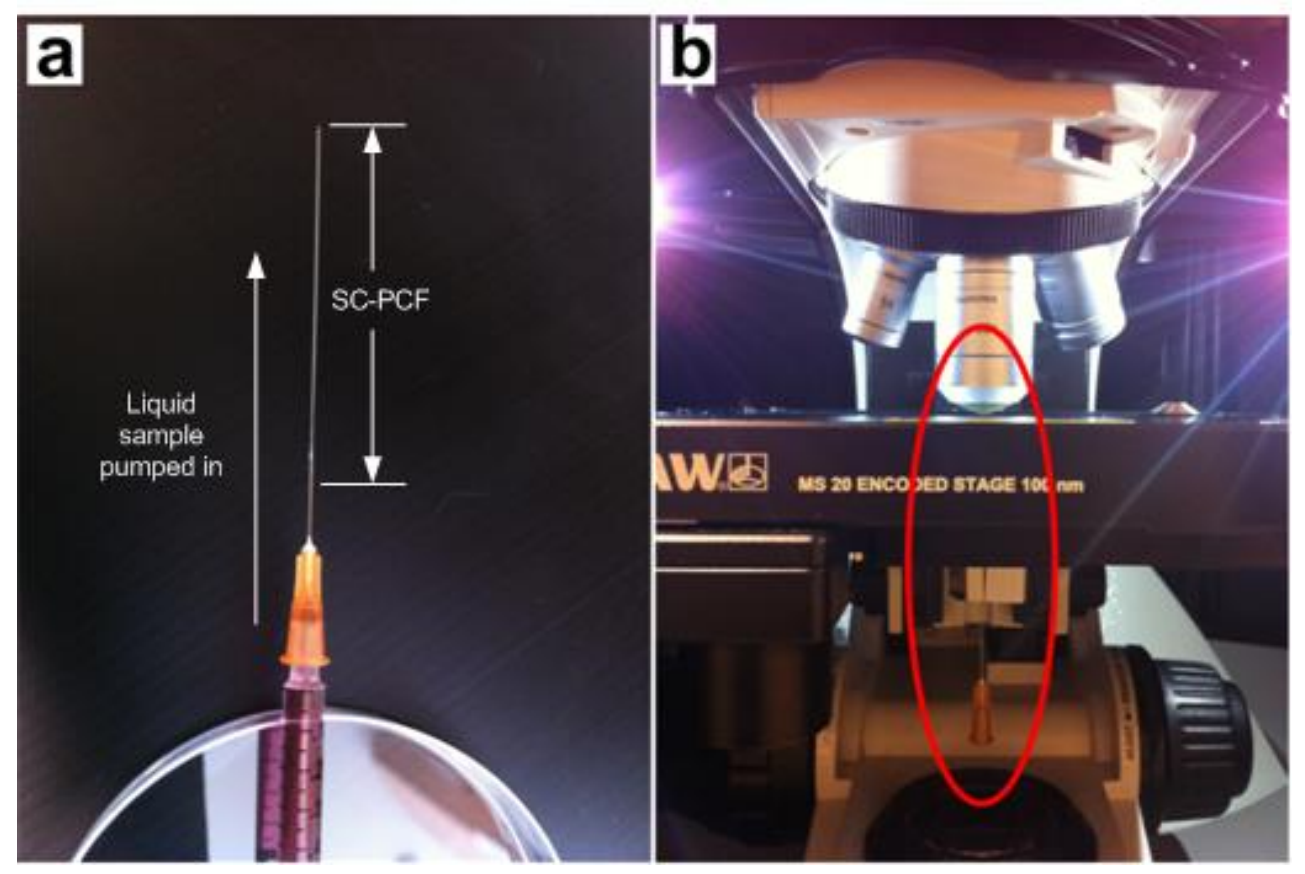

Figure 7.2 SC-PCF based SERS detection setup. (a) Photograph of a syringe connected SC-PCF fiber. The concentration of cells in each sample was fixed at 20,000 cells $/ \mathrm{mL}$ and they were uniformly dispersed when pumped into the fiber. This ensured that our detection of sialic acid was restricted to the single 
cell level. (b) The fiber with the liquid sample pumped in was mounted on the microscope stage using an SMA connector and laser light was coupled into the free-end of the fiber through the objective lens

In this contribution, we designed a side channel photonic crystal fiber (SC-PCF) based platform that is capable of quantifying levels of a specific biomarker on a single cell. With this platform, sensitive detection and quantification of the mean sialic acid level from several single-cell measurements is achieved (see Figure. 7.2 for fiber based SERS detection setup). Our SC-PCF is designed to allow liquid samples to be pumped through a side channel while light is confined and transmitted in the center solid core (Figure. 7.1(e) and Figure. 7.2(a)). It is worth noting that the size of the side channel is designed to be comparative with the diameter of a cell ( $\sim 25$ um by $25 \mathrm{um}$ ), thus permitting single cell loading within the channel and making the SERS detection possible. As opposed to substrate-based SERS detection, the cells were mixed with AuNP solution and pumped into the side channel of the SC-PCF such that each cell was surrounded volumetrically with large amounts of AuNP. More importantly, light transmitted in the solid core is able to interact with the sample in the side channel along the whole length of fiber, greatly increasing the effective enhancement distance. Therefore, this system is able to achieve three-dimensional (3D) SERS enhancement with extremely high sensitivity, which is ideal for single cell analysis. Sialic acid detection was performed with HeLa cells, as they are known to be overexpressed with 
sialic acid receptors[169]. Dark-field imaging was also performed to validate the single cell detection of our developed system. To our knowledge, there was no previous report of using optical fiber as a highly sensitive SERS platform for sialic acid detection on single cell level. In addition, this platform can cater to applications such as gene expression profiling of single cell to study functional heterogeneity of undifferentiated cells [170].

\subsection{Materials and Methods}

\subsubsection{Simulation}

We used COMSOL Multiphysics commercial software to conduct finite element method modeling. A 2D radio frequency (RF) module with perpendicular wave mode analysis was used to calculate the E-field distribution of the fundamental mode in the core when the side channel of the SC-PCF is filled with water. The light wavelength was fixed to $633 \mathrm{~nm}$ and the refractive indices of the silica fiber and water at this wavelength were 1.457 and 1.33 , respectively. A 3D RF module was adopted to calculate the enhancement factor of AuNP with different sizes. The E-field distribution of single AuNP (gold nanosphere) was calculated within a square box, with the port boundary condition set at the top and bottom face of the box and $633 \mathrm{~nm}$ light excitation at the top propagated along the $\mathrm{z}$ axis while the E-field was set to vibrate along the $\mathrm{x}$ axis. The two faces parallel to the E-field (x axis) were set to the perfect magnetic conductor boundary condition and another two faces perpendicular to the E-field were set to the perfect electric conductor 
boundary condition. Given that AuNP was suspended in water in our study, the refractive index of the subdomain surrounding the AuNP was 1.33 while the refractive index of AuNP was set to $0.19683-\mathrm{j} * 3.0905$ [171]. It is generally agreed that the SERS enhancement of AuNP can be approximated by the electromagnetic field enhanced from the LSPR effect. The enhancement factor can be described as follows [172]

$$
E F_{S E R S} \propto \frac{\left|E_{l o c}\left(\omega_{i}\right)\right|^{2}\left|E_{l o c}\left(\omega_{S}\right)\right|^{2}}{\left|E_{0}\left(\omega_{i}\right)\right|^{2}\left|E_{0}\left(\omega_{S}\right)\right|^{2}}
$$

The interaction between incident light and AuNP surface resulted in Raman radiation. With the excitation of Raman-active molecules proportional to the square of the local electric field at the excitation frequency, the radiation can be very high at the surface of metallic structures. $E_{l o c}\left(\omega_{i}\right)$ and $E_{0}\left(\omega_{i}\right)$ refer to the local and incident electric fields at the laser excitation frequency. The emitted Raman radiation was enhanced by the plasmon resonance, which was proportional to the local electric fields at the Raman scattered frequency. $E_{l o c}\left(\omega_{s}\right)$ and $E_{0}\left(\omega_{s}\right)$ refer to the local and incident electric fields at the Raman scattered frequency. In most cases, due to the weak spectral dependence and ease of calculation, the Raman enhancement factor can be calculated as

$$
E F_{S E R S}=\frac{|E|^{4}}{\left|E_{0}\right|^{4}}
$$

where $E$ and $E_{0}$ refer to $E_{l o c}\left(\omega_{i}\right)$ and $E_{0}\left(\omega_{i}\right)$, respectively. 


\subsubsection{SC-PCF Design and Fabrication}

The SC-PCF was fabricated at the XLIM Research Institute under the ICT ASIA project through the stack-n-draw process, during which the silica capillaries were stacked as a preform (hexagonal mesh structure) that was then drawn to a smaller diameter and finally slotted into a prepared tube (outer cladding) and drawn into fiber. As Figure 7.4(a) shows, the fiber was designed with a solid core and six rings of air holes arranged in a triangular lattice; one-sixth of the air holes were combined into a big side hole as the liquid channel with an area of $\sim 660 \mu \mathrm{m}^{2}$. The pitch (distance between adjacent holes) of the fiber was around $6 \mu \mathrm{m}$ and the diameter of the air holes was 3 $\mu \mathrm{m}$ to ensure sufficient evanescent power in the side channel[173] for achieving sensitive detection. The outer side diameter of the fiber was $118 \mu \mathrm{m}$ and the fiber was designed to be an endless single mode fiber to maintain single mode light transmission over the entire range of guidance.

\subsubsection{Fiber Preparation for SERS Detection}

The SC-PCF fibers were cut into 7-centimeter lengths and both ends were cleaved using an optical fiber cleaver. One end of the fiber was plugged into the tip of a $0.3 \mathrm{~mm}$ syringe needle (BD PrecisionGlide) and the connection was sealed with a strong adhesive, ensuring that the liquid sample could be pumped into the fiber through a connected $1 \mathrm{~mL}$ tuberculin syringe (BD PrecisionGlide) for SERS measurement. It is worth noting that the maximum volume of liquid in the side channel of this fiber segment was $\sim 46.2 \mathrm{~nL}$. 


\subsubsection{HeLa Cell Culture and Varying Sialic Acid Levels}

HeLa cells were cultured in Dulbecco's modified eagle medium (DMEM) with $1 \%$ penicillin streptomycin (Gibco) and $10 \%$ fetal bovine serum (Gibco) in the dark with $5 \% \mathrm{CO}_{2}$ at $37^{\circ} \mathrm{C}$ until they were confluent. The cells were then harvested by adding $1 \times$ Ethylenediaminetetraacetic acid (EDTA) trypsin of appropriate volume for $4 \mathrm{~min}$, followed by the addition of DMEM of double that volume to neutralize the trypsin. The harvested cells were centrifuged at 1,500 rpm for $3 \mathrm{~min}$. The supernatant was removed and the cells were suspended with DMEM to achieve a cell concentration of 5000 cells/mL. Two milliliters of cell suspension was seeded per well in a six-well plate and allowed to grow for 48 hours.

After cell proliferation, the DMEM was removed from each well and the adhered cells were rinsed lightly with $1 \times$ PBS, which was subsequently removed. Four wells were used in our experiment. To reduce the probability of DBA binding onto sialic acid sites on the surface of the cell membrane and mimicking a cell with lowered sialic acid level, $10 \mathrm{mM}$ of phenyl-boronic acid (PBA) (Sigma Aldrich) dissolved in DMEM and 1\% Dimethyl sulfoxide (DMSO) (Merck Millipore) were added to one well (Well A) and left to incubate for 24 hours. Five micromolar prednisolone (Sigma Aldrich) dissolved in DMEM and 1\% DMSO was added to another well (Well B) to induce the overexpression of sialic acid, and left to incubate for 72 hours. The remaining two wells (Wells $\mathrm{C}$ and $\mathrm{D}$ ) were used for positive and negative 
controls. Post-incubation washing was performed for all of the wells and 10 mM of DBA (Sigma Aldrich) dissolved in DMEM and 1\% DMSO was added to wells $\mathrm{A}, \mathrm{B}$ and $\mathrm{C}$ for an incubation time of 4 hours. All four wells were washed twice with $1 \times$ PBS to remove excess compounds and unbound cells, and then harvested by trypsinization and centrifugation. The supernatants were removed after centrifugation, leaving the cells ready for SERS sample preparation.

\subsubsection{SERS Sample Preparation}

To evaluate the SERS performance between SC-PCF and HC-PCF, ethanol dissolved 2-Naphthalenethiol (NT) $(5 \mu \mathrm{L}, 100 \mu \mathrm{M})$ was mixed with $495 \mu \mathrm{L}$ of $60 \mathrm{~nm}$ AuNP solution $\left(\sim 10.4 \times 10^{10}\right.$ particles/mL) (see Figure 7.3 for comparison of size dependence of AuNP on SERS enhancement) to obtain an effective concentration of $1 \mu \mathrm{M}$. To evaluate the size dependence of AuNP on SERS performance, $495 \mu \mathrm{L}$ of AuNP of each corresponding size was mixed with DMSO-dissolved DBA $(5 \mu \mathrm{L}, 10 \mathrm{mM})$ separately to obtain DBA of an effective concentration of $100 \mu \mathrm{M}$. The samples for calibration were made in the same manner, but with effective concentrations of DBA at $50 \mathrm{~nm}, 100 \mathrm{nM}$, $500 \mathrm{nM}, 1 \mu \mathrm{M}, 10 \mu \mathrm{M}$ and $100 \mu \mathrm{M}$ in $60 \mathrm{~nm}$ AuNP solution. The four types of sialic acid detection samples were prepared by mixing $60 \mathrm{~nm}$ AuNP solution with the harvested cells from the four wells (A, B, C and D) separately, thus the effective concentration of cells in each sample was 20,000 cells/mL. 


\subsubsection{TEM}

The transmission electron microscope (TEM) was used with JEOL JEM-1010

to characterize the shapes and sizes of the AuNP. The TEM specimens were prepared by instilling $2.5 \mu \mathrm{L}$ of AuNP solution onto 200-mesh nickel-coated grids which were dried prior to use.

\subsubsection{SERS Measurement}

SERS spectra were recorded using a Raman microscope system (Renishaw InVia) with a $633 \mathrm{~nm}$ excitation laser and equipped with a grating (spectral resolution of $\sim 1.8 \mathrm{~cm}^{-1}$ ). A microscope (Leica) and a CCD detector cooled at $-70{ }^{\circ} \mathrm{C}$ were connected to the system. The laser was coupled through a $50 \times$ objective lens, which was used to collect the Stokes-shifted Raman signal. Rayleigh scattering was blocked with a notch filter. In this study, the samples were excited with a laser power of $\sim 3.5 \mathrm{~mW}$. The instrument was calibrated with a silicon standard at a Raman peak of $520 \mathrm{~cm}^{-1}$. The fiber with the liquid sample pumped in was mounted on the microscope stage using an SMA connector and light was coupled into the free-end of the fiber through the objective lens (Figure. 7.2(b)). The SERS signal from the entire length of the fiber was collected in a backscattering geometry.

\subsubsection{Dark-field Imaging}

The HeLa cell and AuNP mixture in the fiber was pumped out by air using an empty syringe, and then loaded onto a glass slide. The samples were visualized using an enhanced dark-field illumination system (CytoViva, 
Auburn, AL) attached to a Nikon LV100 microscope. The system comprised a dark-field condenser (CytoViva) attached via a fiber optic light guide to a 24-watt metal halide light source (Solarc Lighting Technology). Images were acquired at $300 \mathrm{~ms}$ exposure time using a Nikon objective lens $(100 \times$, NA 1.25 and WD 0.23 , oil lens) and a Nikon DS-Fi1 camera with associated software (NIS-Elements D).

\subsection{Results and Discussion}

\subsubsection{Size Dependence of AuNP on SERS enhancement of DBA}

AuNP were used in our SERS detection as they dramatically enhance Raman signals due to their strong LSPR $[40,174]$. As the LSPR wavelength is size dependent, in order to obtain maximum SERS enhancement, we investigated the correlation of DBA intensity enhancement with AuNP of different diameters ranging from 5 to $250 \mathrm{~nm}$ in aqueous solutions. The sample solutions were tested on glass slides and in SC-PCF fiber to compare their enhancement capabilities. As Figure 7.3 (a),(d) shows, AuNP with a diameter of $60 \mathrm{~nm}$ (Figure 7.3(b)) and $100 \mathrm{~nm}$ provides the highest DBA signal enhancement. This experimental result correlates well with our simulation results, as shown in Figure 7.3(d). In our simulation, we only calculated the enhancement factors of single AuNP (as shown in Figure 7.3(c)) without considering the effect of chemical bonds and the hot spots between multiple AuNP. Therefore, there is a slight difference between our calculations and the experimental results that have been previously reported by many other groups 
$[175,176]$. It is worth noting that the signals obtained from the glass slides are much weaker than those of the SC-PCF fiber because laser light is scattered diffusedly after impinging on the glass slide sample, such that only part of the scattered light can be collected by the objective lens. In SC-PCF, however, the scattered light can be conducted back to the objective lens. More importantly, the laser light interacts with the sample solution for a much longer distance along the whole length of fiber than on the glass slide, resulting in much stronger signal intensity.
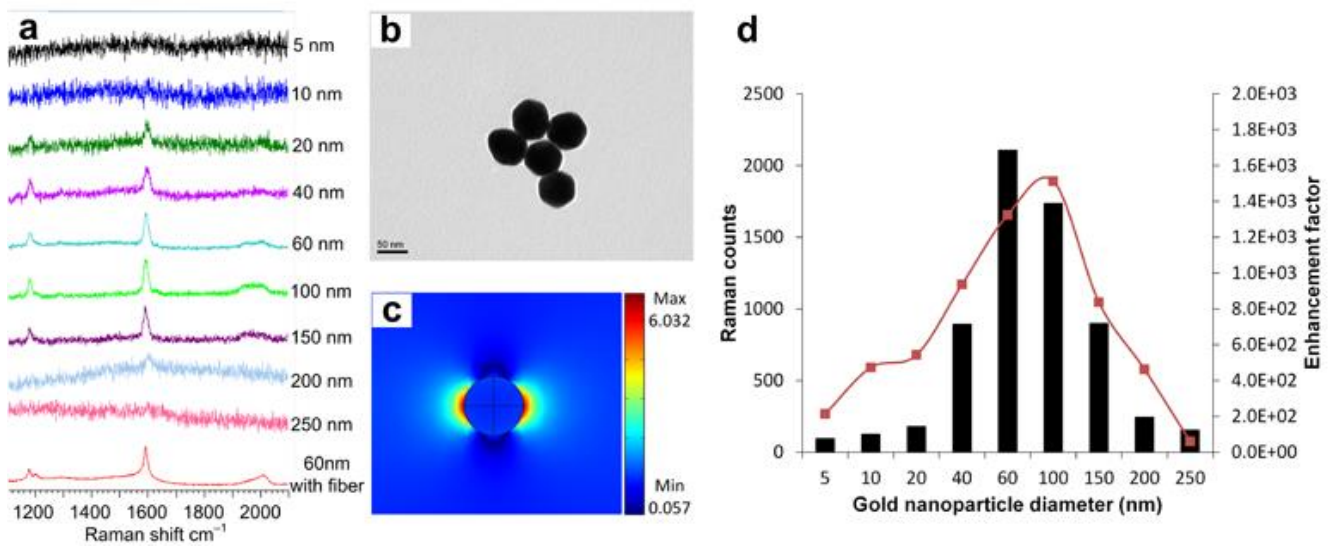

Figure 7.3 Raman enhancement of AuNP with different diameters. (a) Raman spectra of $100 \mu \mathrm{M}$ DBA with different AuNP diameters tested on glass slides and $100 \mu \mathrm{M}$ DBA with $60 \mathrm{~nm}$ AuNP tested in SC-PCF. (b) TEM image of 60 nm AuNP. (c) Calculated $|\mathrm{E}| /\left|\mathrm{E}_{0}\right|$ of $60 \mathrm{~nm}$ AuNP under $633 \mathrm{~nm}$ light excitation. (d) Raman intensity of $100 \mu \mathrm{M}$ DBA with different AuNP diameters (black bar plot) and the calculated Raman enhancement factor for AuNP of different diameters (red line). 


\subsubsection{SERS Enhancement Comparison Between SC-PCF and Conventional HC-PCF}

We first compared the SERS detection sensitivity of SC-PCF (Figure 7.4(a)) and conventional HC-PCF (Figure 7.4(b)) when filled with liquid samples. In the HC-PCF, the air core has a low refractive index relative to the efficient refractive index of cladding, which is not the case in a solid core fiber. Light in $\mathrm{HC}-\mathrm{PCF}$ is confined in the air core due to the band gap effect from its photonic crystal structured cladding, thus light is less confined when the air holes are filled with liquid, resulting in decreased Raman detection sensitivity. In contrast, in SC-PCF, light is confined by the total internal reflection due to the core's higher refractive index (refractive index of silica at $633 \mathrm{~nm}$ is 1.457) relative to the cladding, even when the side channel is filled with water (refractive index 1.33 ). In order to compare the SERS intensity between these two types of fibers, a commonly used Raman tag, 2-Naphthalenethiol (NT), were mixed with 60nm AuNP and pumped into the fiber for Raman detection. As shown in Figure 7.4(e), the Raman signal of NT from SC-PCF is more than six times higher than that in HC-PCF, indicating that our SC-PCF performed much better in liquid sample detection. A simulation was also performed to calculate the electric field (E-field) distribution in the core when the side channel is filled with water. As shown in Figure. 7.4(c) and (d), light is well confined within the core of SC-PCF, which explains the much higher Raman intensity. 

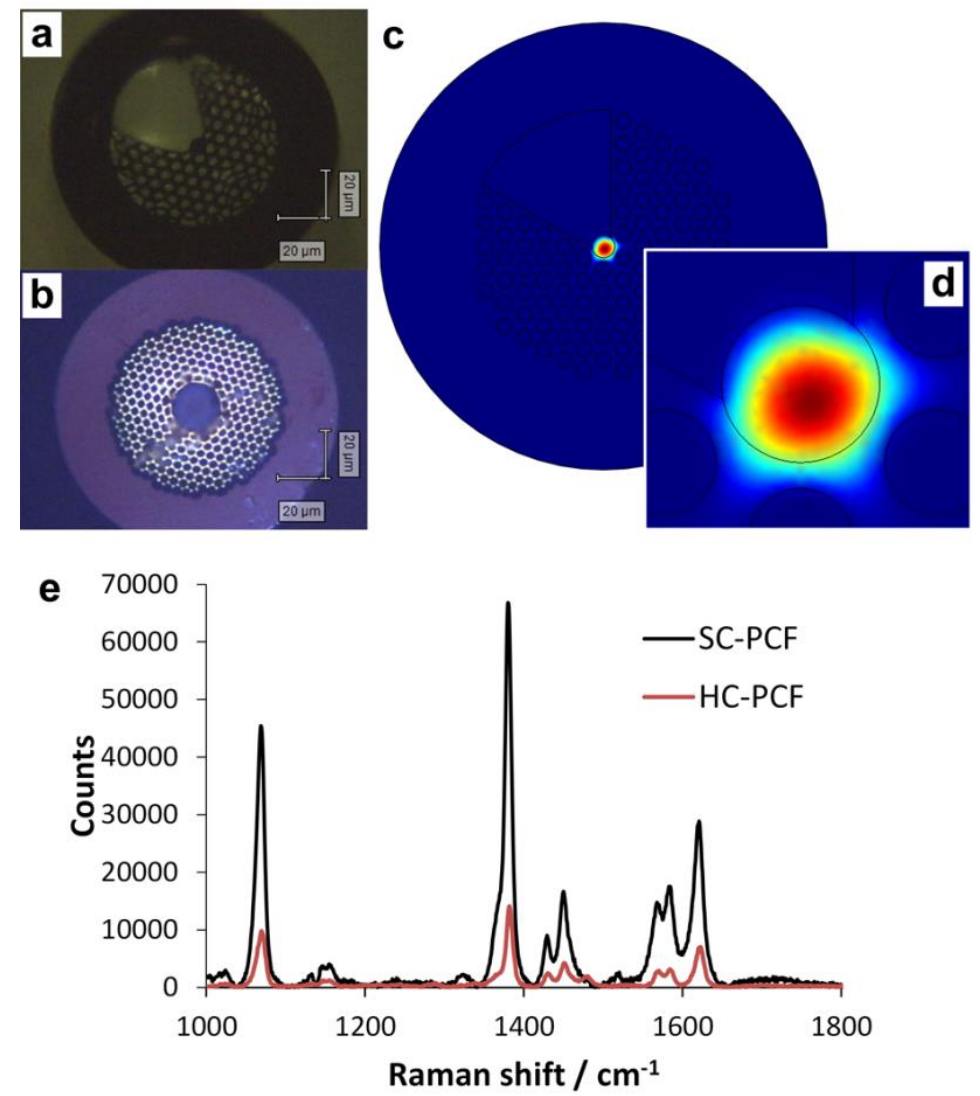

Figure 7.4 Comparison between Raman signal intensity in SC-PCF and HC-PCF. Microscope image of a cross section of (a) SC-PCF and (b) HC-PCF. (c) The calculated E-field distribution of SC-PCF when the side channel is filled with water (wavelength at $633 \mathrm{~nm}$ ). (d) Enlarged image of E-field distribution in fiber core. (e) The Raman spectra of $1 \mu \mathrm{M}$ NT in $60 \mathrm{~nm}$ AuNP solution tested using SC-PCF and HC-PCF

\subsubsection{Correlation of DBA Concentration with SERS Intensity}

Given that the complexing between DBA and sialic acid molecules is unified on the cell membrane, and by evaluating the number of DBA molecules, we were able to quantify the amount of sialic acid. One effective way to quantify sialic acid is to perform a Raman intensity calibration study for DBA with 
different concentrations. As Figure 7.5(a) shows, DBA has three major Raman peaks at $1,174 \mathrm{~cm}^{-1}, 1,592 \mathrm{~cm}^{-1}$ and $2,000 \mathrm{~cm}^{-1}$ that can be potentially used for intensity calibration. However, the Raman signal from cell overlaps with the DBA signal below $1800 \mathrm{~cm}^{-1}$, interfering with the intensities of the DBA peak at $1,174 \mathrm{~cm}^{-1}$ and $1,592 \mathrm{~cm}^{-1}$, which results in an incorrect detection value. In contrast, the DBA peak at $2,000 \mathrm{~cm}^{-1}$ from its alkyne functional group is beyond the aforementioned range and within a relatively clean region. Therefore, it is the peak of choice in our calibration study.

In the calibration study, samples with different DBA concentrations were pumped into the SC-PCF for SERS measurement. As Figure 7.5(b) shows, the Raman intensity of the DBA spectra increased with higher concentrations. It is worth noting that with the strong 3D enhancement from our novel SC-PCF fiber, the detection limit of the $2000 \mathrm{~cm}^{-1}$ DBA peak was as low as $50 \mathrm{nM}$. Therefore, our system makes it possible to achieve highly sensitive detection of sialic acid on single cell. With the amount of sialic acid molecules on cell was estimated from the amount of bonded DBA molecules, we calculated the number of DBA molecules of each calibration sample in the $46.2 \mathrm{~nL}$ side channel of the fiber segment. The calculated numbers of DBA molecules for each sample with DBA concentrations ranging from $50 \mathrm{nM}$ to $100 \mu \mathrm{M}$ in the side channel of the fiber were 2.31 fmoles, 4.62 fmoles, 23.1 fmoles, 46.2 fmoles, 462 fmoles and 4.62 pmoles, correspondingly. 

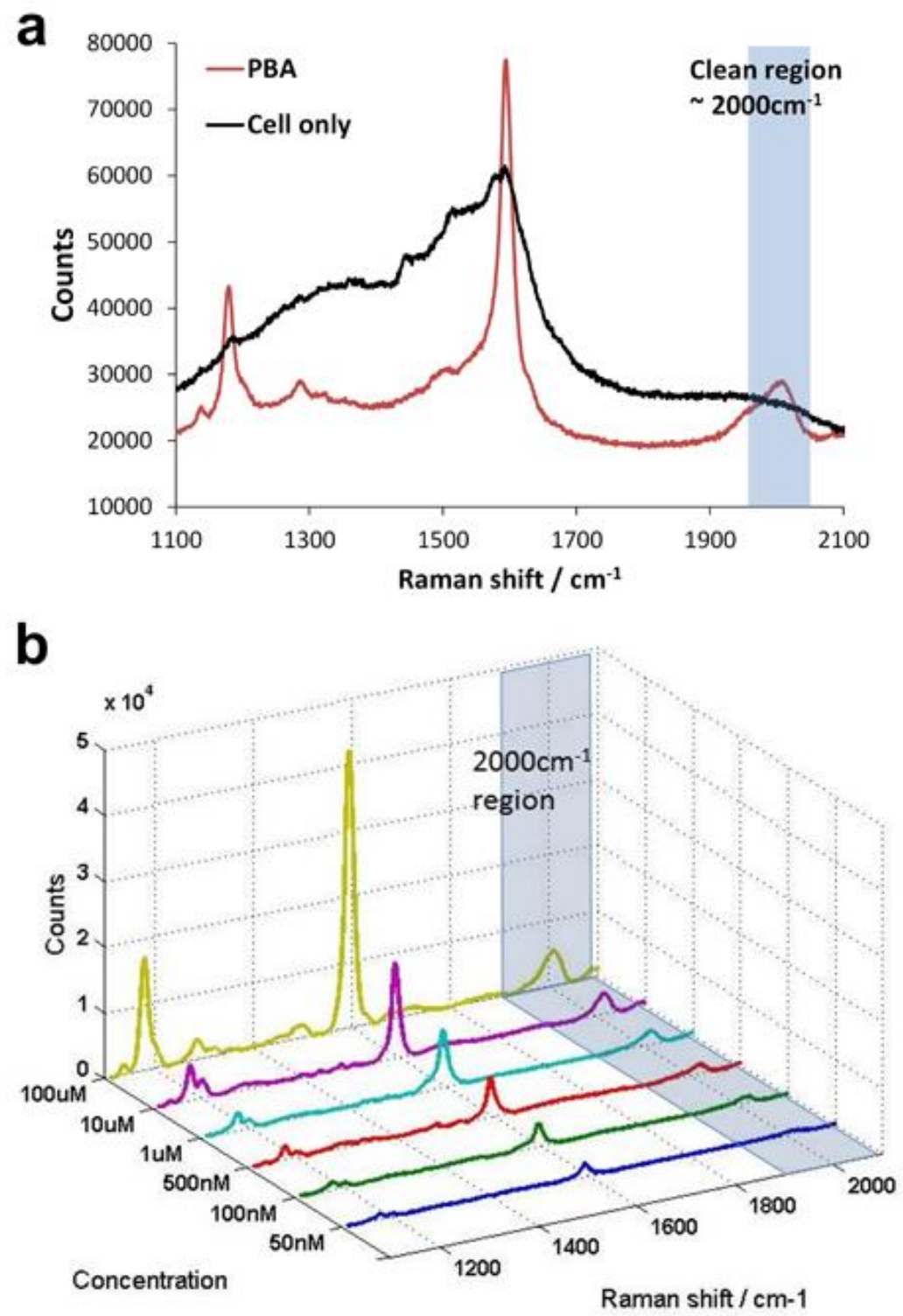

Figure 7.5 (a) Raman spectra of $100 \mu \mathrm{M}$ DBA with $60 \mathrm{~nm}$ AuNP (red line) and HeLa cell (blue line) in SC-PCF. (b) Raman spectra of different concentrations of DBA with $60 \mathrm{~nm}$ AuNP in SC-PCF. $2,000 \mathrm{~cm}^{-1}$ peak intensities for different concentrations of DBA were measured for calibration.

\subsection{Detection and Quantification of Sialic Acid on Single Cell}

To evaluate the sensitivity of our detection in relation to the changes in the sialic acid level, we manipulated the latter by incubating cells in the presence 
of prednisolone (which encourages sialic acid production) and PBA (which binds sialic acid competitively with DBA, depending on the sequence in which the compounds were added to the cells). The sialic acid level in the HeLa cells can be elevated through treatment with prednisolone, a chemical that can inhibit the shedding of surface sialopeptides into the medium[177]. Four cases were studied, namely, HeLa cells successively treated with prednisolone and DBA (herein refers to (+)Sialic acid)), HeLa cells successively treated with PBA and DBA (herein refers to (-)Sialic acid)), normal HeLa cells treated with DBA as positive control (herein refers to Sialic acid) and normal HeLa cells without DBA tag as negative control (herein refers to Cell only). These samples were pumped by syringe into the SC-PCF and mounted on the Raman system stage by a fiber connector, enabling the transmission of laser light into the fiber for SERS detection (Figure 7.2(a),(b)). We fixed the concentration of cells in each sample to be 20,000 cells/mL and they were uniformly dispersed before pumping them into the fiber. The resulting estimated number of cells in a $46.2 \mathrm{~nL}$ side channel of the fiber segment was less than one $(\sim 0.924)$. This ensured that our detection of sialic acid was restricted to the single cell level. To further validate our assumption, we used an empty syringe to pump the liquid sample from the fiber onto a glass slide after SERS measurement. We then used dark-field imaging to exam the number of cells in the sample, as shown in Figure. 7.6 Notably, only one HeLa cell was found in the field. 


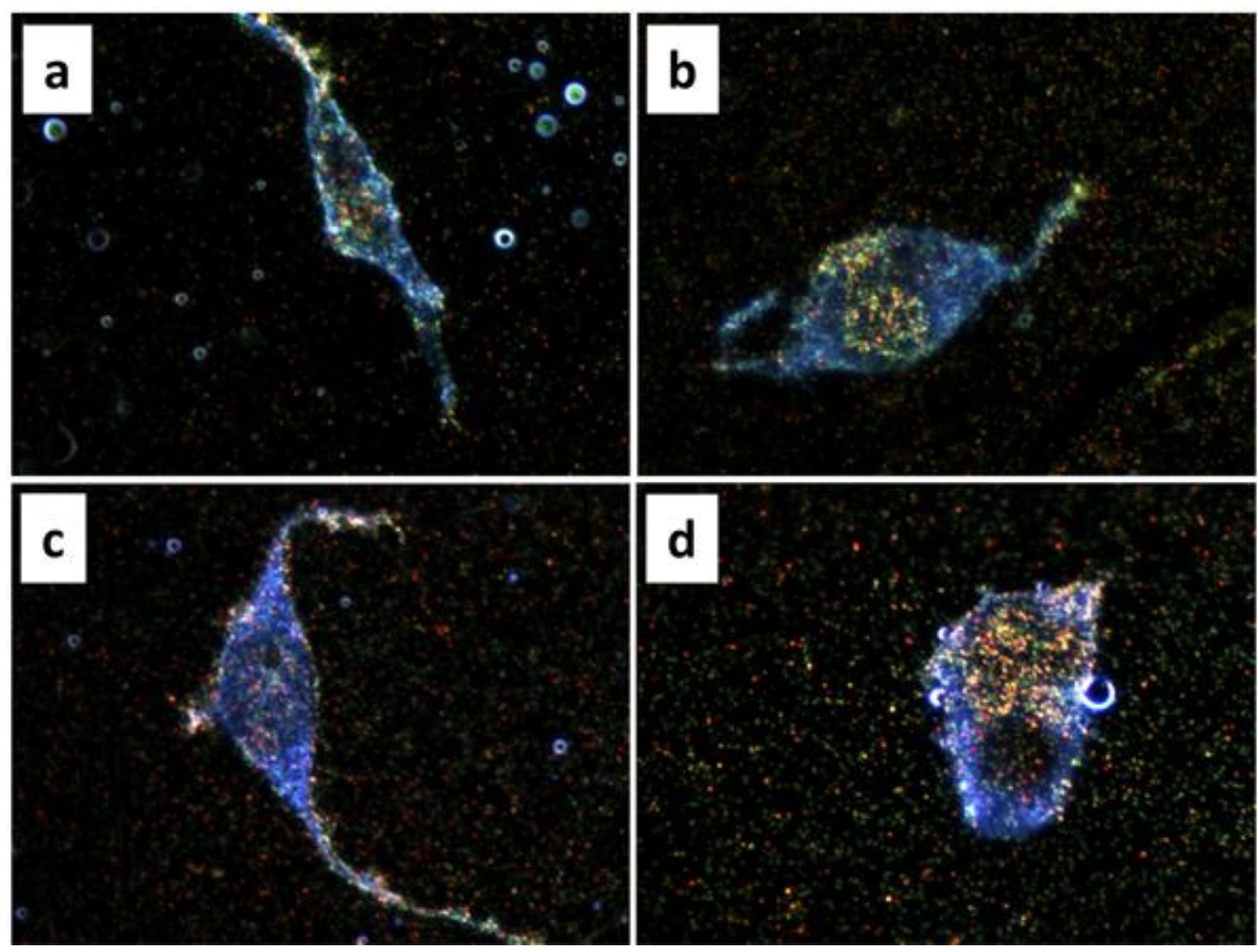

Figure 7.6 Dark-field image of single HeLa cell surrounded by 60nm AuNP pumped out from syringe-connected SC-PCF of four studied cases: (a) A Hela cell successively treated with prednisolone and DBA (herein refers to (+)Sialic acid)). (b) A Hela cell successively treated with PBA and DBA (herein refers to (-)Sialic acid)). (c) A Normal HeLa cell treated with DBA as positive control (herein refers to Sialic acid) and (d) A normal HeLa cell without DBA tag as negative control (herein refers to Cell only).

The Raman spectra of above four cases are shown in Figure 7.7(a). As the DBA bind to the sialic acid expressed by HeLa cell, a 2,000 $\mathrm{cm}^{-1}$ peak of DBA is observable for positive control sample (Sialic acid). The intensity of $2,000 \mathrm{~cm}^{-1}$ peak for the cell treated with prednisolone was observed to be higher than that of the positive control, suggesting increased sialic acid 
expression in the treated cell. Given the similarity in chemical structure between phenylboronic acid and DBA, both will bind to sialic acid. Thus, after pre-treatment with PBA in the (-)Sialic acid sample, the probability of DBA binding to sialic acid on HeLa cells is lowered. Because DBA contains an alkyne functional group that gives rise to the $2,000 \mathrm{~cm}^{-1}$ peak, the negligible presence of that peak in the Raman spectrum of the (-)Sialic acid sample does not indicate the binding of DBA. The spectrum of the negative control was shown to overlap with that of DBA at wavenumbers below 1,800 $\mathrm{cm}^{-1}$, confirming that the intensity of the $2,000 \mathrm{~cm}^{-1}$ DBA peak was uninterrupted. A spectrum was obtained where no cell was loaded into the fiber as a result of their low concentration (and consequently, a lowered probability of observing a cell). Here, no peak was observed in the entire spectrum.

To calculate the amount of sialic acid on a cell in the Sialic acid and (+)Sialic acid samples, the intensity of the $2,000 \mathrm{~cm}^{-1}$ peak was obtained from our SERS measurements (Figure 7.7(b)). By using the calculated numbers of DBA molecules and their corresponding intensities obtained from above study, we plotted a calibration trend line. This trend line clearly represented the correlation between the intensity of the $2,000 \mathrm{~cm}^{-1}$ peak and the number of DBA molecules (black line and dots in Figure 7.7(c)). The number of sialic acid molecules was quantified by estimating the amount of DBA molecules from the trend line, as the binding between DBA and sialic acid molecules is 
assumed to be one-by-one. We estimated the number of DBA molecules from the calibration curve, and the calculated mean sialic acid amounts on single HeLa cell based on several single-cell measurements for Sialic acid and (+)Sialic acid samples were 2.514 fmoles and 8.286 fmoles, respectively. This result correlates well with previously reported results [152-154] in which the values of sialic acid molecules on single cell were derived by calculating the mean value originated from large amount of cells (from $10^{5}$ to $10^{9}$ cells). In addition, a control experiment using commercial sialic acid quantitation kit (Sigma Aldrich) was performed to quantify the sialic acid level in Sialic acid and (+)Sialic acid samples. In brief, cells were treated with neuraminidase to release sialic acid from surface of cells and then removed by centrifugation. Free sialic acid in the supernatant was converted to pyruvic acid by $\mathrm{N}$-acetylneuraminic acid aldolase, and then treated with lactic dehydrogenase to form lactic acid with the oxidation of $\beta$-NADH to $\beta$-NAD. The values of sialic acid molecules were calculated based on the $340 \mathrm{~nm}$ absorbance peak read from spectrophotometer. The amount of sialic acid molecules in Sialic acid and (+)Sialic acid samples were found to be 1.447 and 4.502 nmoles in $10^{6}$ cells, which corresponded well to those obtained using our SERS study. The current available methods for detecting sialic acid on cells mainly relied on colorimetric and fluorometric based sensors. For example, Warren et al. originally reported the use of colorimetric based sensor together with thiobarbituric acid for sensing sialic acid on tissues [178]. Later, Prasanta et 
al. proposed the quantification of sialic acid from normal and malignant cells using similar colorimetric based approach [152]. However, this method cannot
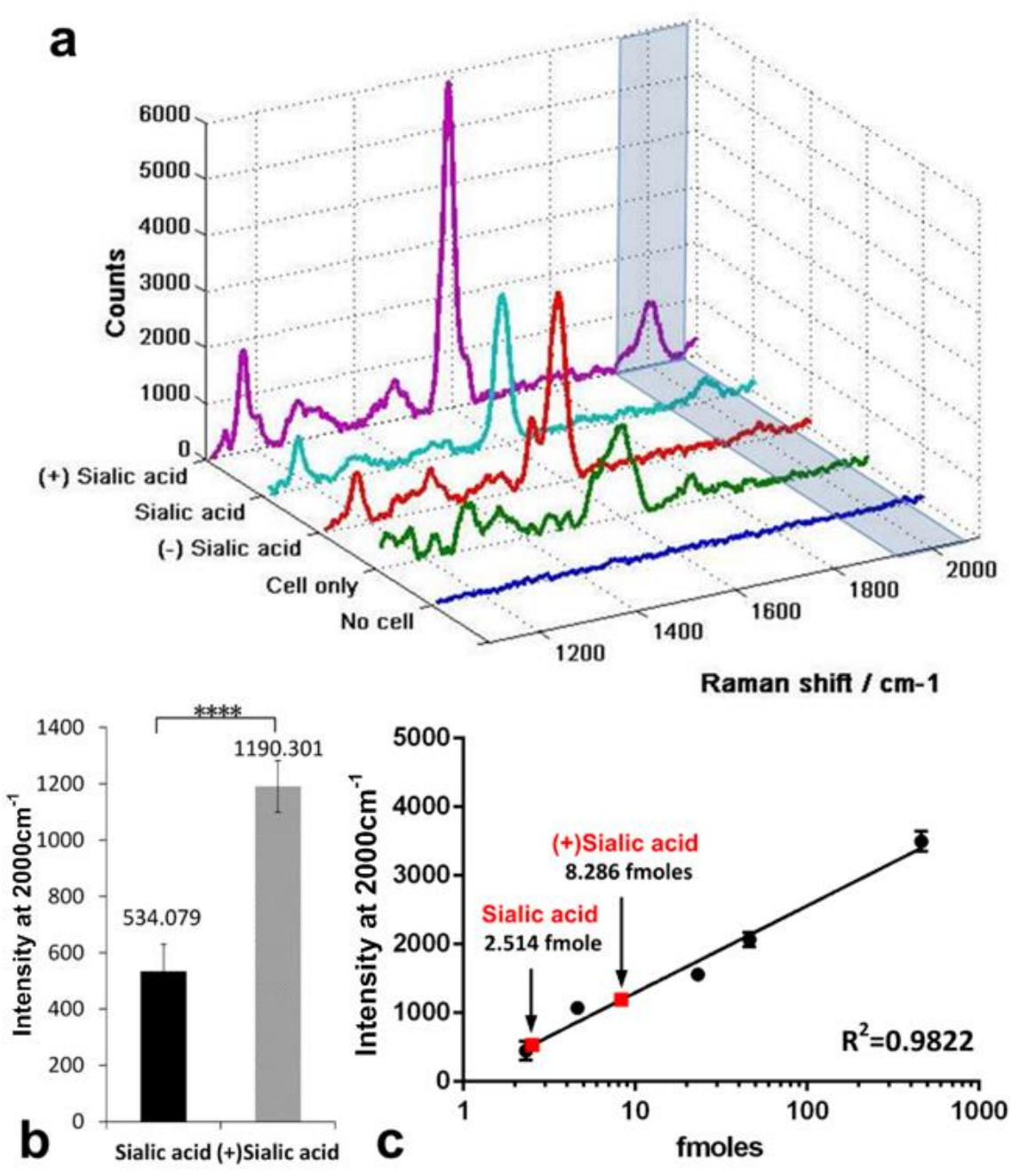

Figure 7.7 SERS detection and quantification of single Hela cell. (a) Raman spectra of (+)Sialic acid sample, (-)Sialic acid sample, positive control (Sialic acid), negative control (Cell only) and Raman signal from the fiber when no cell was loaded. (b) $2000 \mathrm{~cm}-1$ peak intensity of (+)Sialic acid and Sialic acid samples. (c) Calculated number of sialic acid molecules (red square for Sialic acid and (+)Sialic acid samples) from plotted trend line (black line) of the calibration points (black dots) (The $\mathrm{x}$ axis is in log scale). 
be performed with living cells, as acid reagent and high temperature is required to obtain colored solution, and this will kill the cells. More recently, Jessie et al. employed fluorometric based sensor and a carbonyl-reactive chromophore for sensing sialic acid[179]. However, the synthesis of the chromophore is complex and tedious and most importantly the maximum fluorescence enhancement signals can only be obtained for cell labelling condition under $\mathrm{pH}$ 4.7. Such approach is not suitable for long term investigation on the dynamic interaction between the sialic acids and the cells. Akira et al. reported the use of PBA modified gold electrode for detecting sialic acid on the cell membrane [147] and this is a relatively more practical approach as compared to other methods. However, this method requires additional steps for modifying electrode with PBA using 10-carboxy-1-decanethiol, and more than a million cells are needed for the detection purpose. This approach, in general, is still considered to be inappropriate and insufficient to be used for detecting sialic acid with limited amount of cells.

Apart from practical limitations of the previously reported techniques, sensitivity must also be considered. In an example that employed colorimetric technique, P. K. Ray et al. found the levels of sialic acid ranging from 4.6 to 500 nmoles in $10^{9}$ of various normal or malignant mouse cells [152]. T. Kawai et al. reported a more sensitive method using chromatography-mass spectrometry which was capable of a low detection limit of 0.12 pmol. In their 
work, they detected sialic acid levels ranging from 9.7 to 57.8 nmoles in $1 \mathrm{mg}$ of glycosphingolipid in different human cancer tissues [145]. Recently, Y. Zhou et al established a quicker technique by means of potentiometry with a detection limit of $0.8 \mu \mathrm{M}$ to directly determine sialic acid level. In their work, they could detect in the range of $2 \mu \mathrm{M}$ to $1.38 \mathrm{mM}$ and also demonstrated the technique's application on human blood serum [180]. There are also commercially available fluorescence/absorbance-based kits that typically measure in the linear range of 0.1 to 10 nmoles (Abcam) or 1 to 200 nmoles (Sigma-Aldrich) with detection sensivity of $\sim 1 \mu \mathrm{M}$. In comparison to the mentioned methods above, our SERS based SC-PCF detection method is simple and flexible to detect sialic acid on single cell with high detection sensitivity in femto molar.

\subsection{Summary}

In conclusion, we successfully demonstrated a novel SERS-based platform that can detect and quantify the average sialic acid on single cancer cell using a SC-PCF-based system. To the best of our knowledge, this is the first reported SERS-based fiber platform that accurately quantifies sialic acid at the single cell level. Compared with previously reported sialic acid detection methods, our method is cost-effective and practical. More importantly, with the benefits of SC-PCF's liquid sample detection capabilities, combined with a non-interfered 2,000 $\mathrm{cm}^{-1}$ DBA peak and the ability of AuNP to provide 
strong 3D SERS enhancement, we were able to achieve highly sensitive detection of compounds on a single cell. Given this combination of advantages, we expect that our system can not only be used in sialic acid detection, but also developed into a clinical diagnostic tool with broad applications in the highly sensitive detection of biomolecules from various types of liquid samples such as blood, serum, plasma, urine, saliva, sweat and tears. 


\section{Chapter 8 Sensitive SERS Multiplexed Detection of Matrix Metalloproteinase 2 and 7 Cancer Markers}

\subsection{Introduction}

Matrix metalloproteinase are a family of zinc-dependent endopeptidases. They play a part in the proteolytic degradation of the components of extracellular matrix (ECM) and infiltration through the basal membrane, alteration of cell-cell and cell-ECM interactions, migration and angiogenesis, bringing to light their importance in tumor growth and the multi-step processes of invasion and metastasis [181, 182]. More than 26 members of secreted and membrane bound matrix metalloproteinases (MMPs) have been found and extensively studied for their roles in cancer in the past decades. Particularly, it was found that the active form of MMP-2 is significantly elevated in the ovarian tumor compared with the latent form. This could suggest two scenarios: tumor cells with MMP-2 activity are more likely to metastasize, or ovarian tumor cells produce more active MMP-2 after metastasis [183-185]. MMP-2 was also found to be highly expressed in breast cancer tissue compared to normal breast tissue, and therefore could be associated with breast cancer development [186-188]. Previous studies show that the elevation in serum level of MMP-2 in patients with advanced urothelial carcinoma was significantly higher than that in patients with 
superficial bladder carcinoma. Therefore, the serum levels of MMP-2 could be good indicator of invasion and metastasis [189, 190]. Apart from MMP-2, MMP-7 was reported to be expressed in the cytoplasm or cell membrane of esophageal squamous cell carcinoma cells, whereas MMP-2 was essentially absent [191]. MMP-7 expression was found to be significantly related to nodal metastasis and unfavorable prognosis in superficial esophageal cancer. Therefore, the level of MMP-7 in tumor biopsy specimens serves to be a marker for treatment course decision and prognosis prediction [192, 193]. Furthermore, both MMP-2 and MMP-7's overexpression have been reported to correlate closely with carcinomas such as lung cancer [194-196], pancreatic cancer [197, 198], gastric cancer [199, 200] and prognosis of colorectal carcinoma [201-203]. There is thus a great need for simple, rapid, sensitive and multiplexed detection of these MMPs at clinically relevant concentrations for cancer diagnostics and therapeutics.

Many approaches have been developed to detect MMPs, including gel zymography [204, 205], enzyme-linked immunosorbent assay (ELISA) [206], fluorescence resonance energy transfer (FRET) [207], and colorimetric method [208]. Although sensitive, gel zymography and ELISA typically require long and tedious preparations, and are restricted in their applications due to low-throughput assaying. FRET detects MMPs based on tagging a fluorophore and quenching pair on the opposite ends of the peptide. The two ends separate upon MMP enzyme cleavage of the peptide, resulting in 
intensified fluorescence intensity. However, FRET possesses the inherent problem of photo-bleaching in molecular probes and high toxicity associated with the use of quantum dots. In colorimetry, Peng Chen et al. demonstrated its use for MMP-7 detection with a detection limit of $0.1 \mu \mathrm{g} / \mathrm{mL}$ [208]. However, as MMP physiological concentrations in respective carcinomas are between a few $\mathrm{ng} / \mathrm{mL}$ to $\mu \mathrm{g} / \mathrm{mL}[188,189,209,210]$, this method is not sensitive enough, especially for early stage cancer diagnosis. Furthermore, as there are several types of carcinomas that correlate with multiple MMPs, achieving accurate analysis and diagnosis require monitoring of the concentrations of multiple MMPs level simultaneously.

To address these issues, we proposed a SERS based sensing platform to achieve sensitive multiplexed detection of MMP-2 and MMP-7 proteases. This platform adopts a SERS-active planar substrate known as bimetallic-film-over-nanosphere (BMFON) [211]. It comprises multiple nanostructures formed by depositing polystyrene nanospheres onto a glass slide, followed by systematically sputtering a silver (Ag) underlayer and gold $(\mathrm{Au})$ overlayer to impart a gold-silver bimetallic coat. In contrast, the classical film over nanosphere (FONs) are single-layered in nature, and are typically made of silver for its strongly SPR/SERS enhancing property. However, Ag is susceptible to fast atmospheric oxidation. The merit of BMFON lies in retaining this property while insulating it from oxidation with a gold over-coating. More importantly, the outer Au layer is also advantageous to bio 
sensing because of its binding affinity to thiol groups that assist in bioconjugation, The BMFON substrate was first functionalized with biotin-PEG-thiol through thiol-gold bonding. NeutrAvidin was then conjugated to the substrate through strong biotin-avidin binding. In order to detect MMP-2 and MMP-7 enzymes, enzyme-specific MMP-2 (G-K(TAMRA)-G-P-L-G\#-V-R-G-C-CONH2) [212] and MMP-7 (G-K(TAMRA)-G-V-P-L-S\#-L-T-M-G-C-CONH2) [213] (the cleaving sites are marked by \#) peptides were adopted. As both peptides have thiol-containing amino acids cysteine (C), BMFON substrate's active surface can be conjugated with MMP-2 and MMP-7 peptides through thiol-gold interaction [214]. Two types of biotinylated AuNPs were prepared, of which one was tagged with 4-aminothiophenol (ATP), and the other with 2-naphthalenethiol (NT). ATP and NT are commonly used SERS reporter molecules which can provide strong unique SERS peaks at $1584 \mathrm{~cm}^{-1}$ and $1377 \mathrm{~cm}^{-1}$, respectively, from their $v(\mathrm{CC})$ ring stretch $[215,216]$. The ATP-tagged and NT-tagged AuNPs were then conjugated with MMP-2 and MMP-7 peptide, respectively. The detection is based on the virtue of enzymatic reaction where a peptide can be cleaved only by its corresponding enzyme. To perform detection, these AuNPs were loaded onto the BMFON substrate, and the binding between AuNPs and BMFON via biotin-avidin interaction is initially blocked by long chains of the MMP peptide present on AuNPs and substrate that hinder such binding. The binding of AuNPs on 
BMFON can only been observed after peptide chain cleavage by presence of corresponding MMP enzyme (the scheme of detection mechanism were shown in Figure 8.1). As the number of AuNPs binding on BMFON is proportional to the amount of peptide cleavage, the concentration of the MMP enzyme can be quantified by monitoring the SERS intensity of Raman tag on AuNPs. Since the type of AuNP that binds on BMFON is enzyme-specific, multiplexed detection of MMP-2 and MMP-7 enzymes can be achieved by identifying the signature peaks of the Raman tags and noting their intensities.

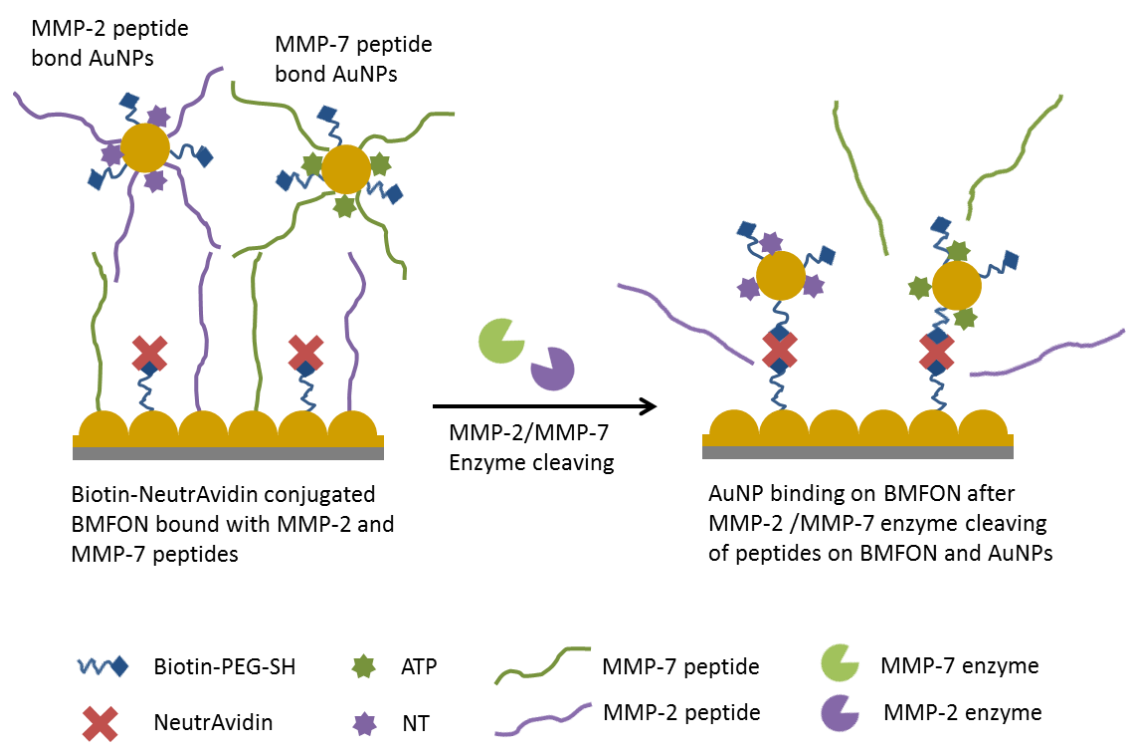

Figure 8.1 Schematic illustration of tagged AuNPs biding on BMFON after MMP-2/MMP-7 enzyme cleaving of peptides on BMFON and AuNPs.

\subsection{Materials and Methods}

\subsubsection{BMFON Substrate Fabrication}

Clean microscope glass slides were cut into $10 \mathrm{~mm} \times 10 \mathrm{~mm}$ squares. They were then sonicated in a bath of ethanol for $20 \mathrm{~min}$ and dried before using. $400 \mu \mathrm{L}$ 
of monodispersed polystyrene (PS) colloidal suspension $(\varnothing=400 \mathrm{~nm}, 2.5 \mathrm{wt} \%$, Kisker) was centrifuged at 7,000 rpm for 10 mins. After removing the clear supernatant, $50 \mu \mathrm{L}$ of water and $50 \mu \mathrm{L}$ of surfactant sodium dodecyl sulfate (SDS) (15 wt \%, Sigma Aldrich) were added to form a composite solution. To form a monolayer of closely packed PS spheres on the glass slides, $5 \mu \mathrm{L}$ of the prepared colloidal solution was loaded onto the center of each glass slide. Each glass slide was spin coated at 2,000 rpm for $20 \mathrm{~s}$ and placed in a desiccator overnight at 0.6 Pa. Each glass slide was then sputter-coated with $80 \mathrm{~nm}$ sublayer of Ag and 40nm of Au overlayer using JEOL JFC-1600 auto fine coater.

\subsubsection{Conjugation of BMFON}

Substrates were first incubated in $50 \mu \mathrm{M}$ biotin PEG thiol (Nanocs) solution for 30 mins and rinsed with water. $15 \mu \mathrm{L}$ of NeutrAvidin $(5 \mathrm{mg} / \mathrm{mL}$ in PBS solution, Life technologies) was subsequently dropped onto the SERS-active surface and incubated for 3 hrs. $1 \mathrm{mM}$ of MMP-2 peptide (G-K(TAMRA)-G-P-L-G-V-R-G-C-CONH 2 ) and $1 \mathrm{mM}$ of MMP-7 peptide (G-K(TAMRA)-G-V-P-L-S-L-T-M-G-C-CONH ${ }_{2}$ ) were dissolved in $1 \times$ phospho-buffered saline and 0.005M EDTA (pH 7.4) separately. As both peptides have thiol-containing amino acids cysteine $(\mathrm{C})$, these peptides will therefore bind to BMFON through thiol-gold interaction. For initial verification, MMP-2- and MMP-7-conjugated BMFON substrates were prepared by incubating biotin-avidin conjugated BMFON substrates in 
MMP-2 and MMP-7 peptide solution, respectively. In order to perform multiplexed detection, BMFON substrate conjugated with a mixture of MMP-2 and MMP-7 were prepared by incubating biotin-avidin conjugated BMFON in solution containing 50\% of MMP-2 and 50\% of MMP-7 peptides. After $3 \mathrm{hrs}$ incubation, these BMFON substrates were rinsed with water to remove unbound peptide before use.

\subsubsection{AuNPs Preparation and Characterization}

For initial verification, two types of AuNPs were prepared.

ATP tagged AuNPs: 1.9 mL of 60 nm diameter AuNPs (OD=1.19 @ 520 nm, $2.60 \times 10^{10}$ particles/mL, BBI Solutions) were mixed with biotin PEG thiol (50 $\mu \mathrm{L}, 20 \mu \mathrm{M})$ and $\mathrm{ATP}(50 \mu \mathrm{L}, 10 \mu \mathrm{M})$ for 15 mins and centrifuged twice at $8,500 \mathrm{rpm}$ for $1 \mathrm{~min}$. After removing the supernatant, water was added to make a final volume of $200 \mu \mathrm{L}$.

NT tagged AuNPs: The AuNPs were prepared in the same process as described above but with NT $(50 \mu \mathrm{L}, 30 \mu \mathrm{M})$ instead of ATP and centrifuged at 9,000 rpm.

For multiplexed detection of MMP-2 and MMP-7 peptides, two types of AuNPs were prepared.

MMP-2 conjugated AuNPs: NT tagged AuNPs were prepared as described above, but with a single round of centrifugation was applied. After removal of the supernatant, $1.9 \mathrm{~mL}$ of water and $0.25 \mu \mathrm{L}$ of $1 \mathrm{mM}$ MMP-2 peptide were 
mixed with the AuNPs for 2 hrs. Following centrifugation, excess MMP-2 peptides were washed away and water was added to make a final volume of $200 \mu \mathrm{L}$.

MMP-7 conjugated AuNPs: MMP-7 conjugated AuNPs was prepared in the same process as described above but with ATP tagged AuNPs and MMP-7 peptide.

Hydrodynamic diameters of these AuNP were studied by using a DLS system (Zetasizer Nano series, Malvern).

\subsubsection{Detection of Individual MMP Enzyme}

MMP-2 and MMP-7 enzymes $(0.1 \mathrm{mg} / \mathrm{mL}$, Sigma Aldrich) were diluted in $0.001 \mu \mathrm{g} / \mathrm{mL}, 0.01 \mu \mathrm{g} / \mathrm{mL}, 0.1 \mu \mathrm{g} / \mathrm{mL}, 0.5 \mu \mathrm{g} / \mathrm{mL}, 1 \mu \mathrm{g} / \mathrm{mL}, 5 \mu \mathrm{g} / \mathrm{mL}, 10$ $\mu \mathrm{g} / \mathrm{mL}, 20 \mu \mathrm{g} / \mathrm{mL}, 30 \mu \mathrm{g} / \mathrm{mL}$ and $40 \mu \mathrm{g} / \mathrm{mL}$ using buffered solutions containing $50 \mathrm{mM}$ Tris- $\mathrm{Cl}, 5 \mathrm{mM} \mathrm{CaCl} 2$ and $0.005 \%$ Brij-35 at $\mathrm{pH} 7.4 . \mathrm{A}$ buffered solution containing no enzyme was used as control.

MMP-2 enzyme detection: MMP-2 conjugated BMFON substrate was incubated with $100 \mu \mathrm{L}$ of MMP-2 enzyme solution at $37^{\circ} \mathrm{C}$ for $3 \mathrm{hrs}$. The substrates were then rinsed with water and $20 \mu \mathrm{L}$ of NT tagged AuNPs was loaded. After incubation for $2 \mathrm{hrs,} \mathrm{the} \mathrm{substrates} \mathrm{were} \mathrm{rinsed} \mathrm{again} \mathrm{to} \mathrm{remove}$ unbound AuNPs, SERS measurement was then performed.

MMP-7 enzyme detection: The same process as described above was carried out using MMP-7 conjugated BMFON substrate and ATP tagged AuNPs. 


\subsubsection{SEM Sample Preparation}

Three BMFON substrate samples were prepared.

Clean BMFON substrate: newly prepared BMFON substrate without any conjugation.

MMP-7 conjugated BMFON substrate: as described above

AuNPs bound BMFON: prepared as same process as described in MMP-7 enzyme detection, were $20 \mu \mathrm{g} / \mathrm{mL}$ of MMP-7 enzyme was used.

\subsubsection{Multiplexed Detection of MMP-2 and MMP-7 enzymes}

Four types of enzymes were prepared: $0.5 \mu \mathrm{L}$ of MMP-2 $(0.1 \mathrm{mg} / \mathrm{mL}), 0.5 \mu \mathrm{L}$ of MMP-7 $(0.1 \mathrm{mg} / \mathrm{mL}), 0.5 \mu \mathrm{L}$ solution contain $0.25 \mu \mathrm{L}$ of MMP-2 $(0.1$ $\mathrm{mg} / \mathrm{mL})$ and $0.25 \mu \mathrm{L}$ of MMP-7 $(0.1 \mathrm{mg} / \mathrm{mL}), 0.5 \mu \mathrm{L}$ of buffer solution as control.

Each of MMP-2- and MMP-7-conjugated BMFON substrate were place in solution containing $50 \mu \mathrm{L}$ of MMP-2 conjugated AuNPs, $50 \mu \mathrm{L}$ of MMP-7 conjugated AuNPs and $0.5 \mu \mathrm{L}$ of enzyme solution. After 2 hrs of incubation at $37^{\circ} \mathrm{C}$, the substrates were rinsed with water and performed with SERS measurement.

\subsubsection{SERS Measurement}

SERS spectra were obtained using a Raman microscope system (Renishaw InVia) with a Peltier cooled CCD detector and a laser excitation wavelength at $785 \mathrm{~nm}$. The laser was coupled through a 50× objective lens, which was used 
to collect the Stokes-shifted Raman signal. Rayleigh scattering was blocked with a notch filter. In this study, the samples were excited with a laser power of $\sim 1.09 \mathrm{~mW}$ with $10 \mathrm{~s}$ exposure time throughout the measurements. The instrument was calibrated with a silicon standard at a Raman peak of 520 $\mathrm{cm}^{-1}$.

\subsection{Results and Discussion}

\subsubsection{Characterization of The BMFON}

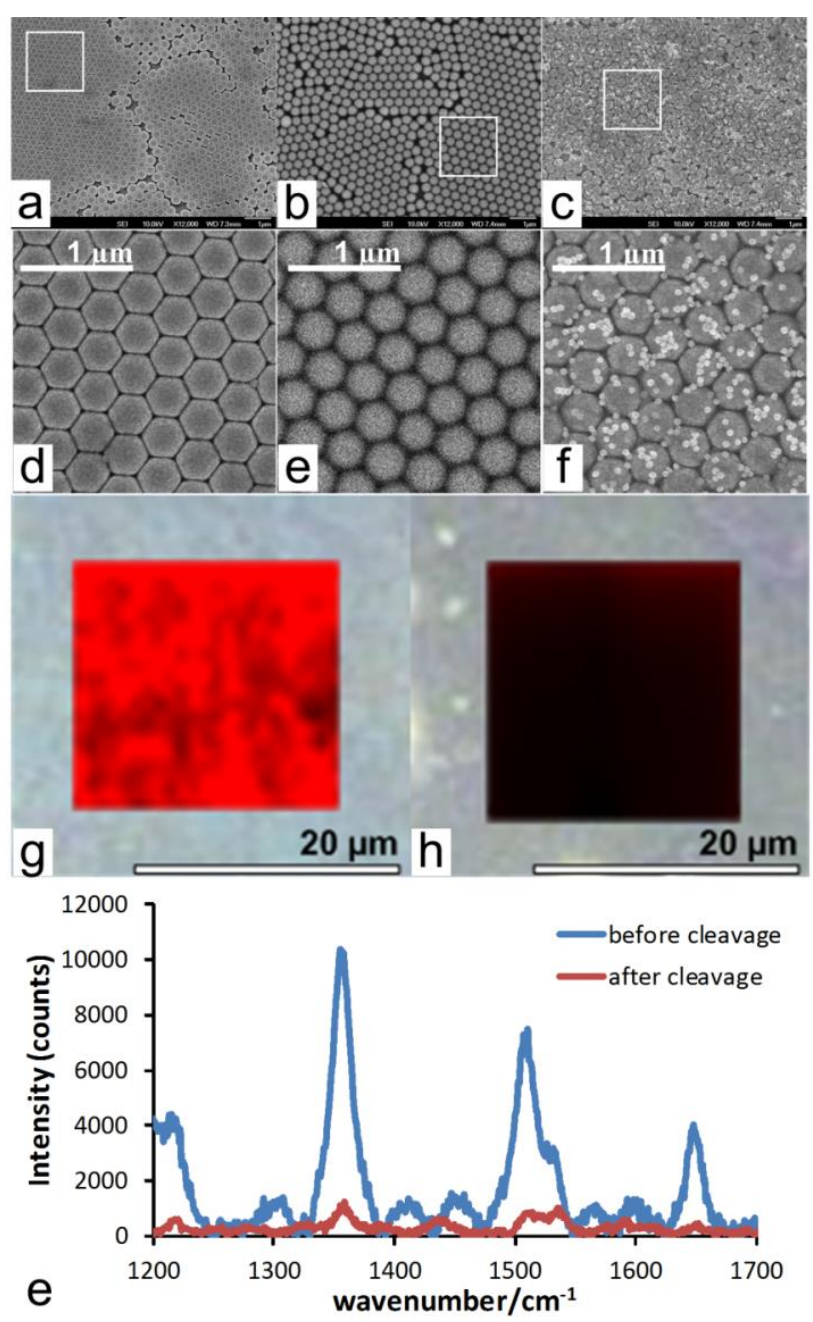

Figure 8.2 BMFON characterization. SEM imaging of (a) clean BMFON substrate (b) peptide shielded biotin-avidin conjugated BMFON and (c) 
AuNPs bond BMFON after enzyme cleaving. (d)(e)(f) Enlargement of images from the area (area within white squares) in (a)(b)(c), respectively.(g) SERS mapping of peptide conjugated BMFON. (h) SERS mapping of peptide conjugated BMFON after enzyme cleavage. (e) Raman spectra of MMP peptide bound BMFON substrate before and after enzyme cleaving.

To evaluate the cleave-and-bind mechanism of the enzyme detection platform, SEM imaging was performed on the unfunctionalized BMFON substrate, MMP-7 conjugated BMFON substrate, and AuNPs-bound BMFON. As shown in Figure 8.2(a) and (d), a large area of monolayer of hexagonally and closely packed spheres was formed on the glass slide due to the self-assembly mechanism. These metal-coated nano-structures are capable of generating plasmonic hot spots in the crevices between spheres upon laser excitation, thus strongly enhancing the Raman signal from molecules on the substrate. The SEM images of peptide conjugated BMFON substrate were shown in Figure 8.2(b) and (e). It is clearly observed that there is a thin layer of peptide coated on the substrate, resulting in a fuzzy image of the nano-structures. This layer of peptide serves to shield the substrate from binding of AuNPs. To understand the relative distribution of peptide on substrates, we carried out SERS mapping after incorporating peptide to substrates. In order to generate Raman signal from peptide, we embedded a Carboxytetramethylrhodamine (TAMRA) segment in MMP-2 and MMP-7 peptide, allowing the distribution of peptide to be examinable by SERS mapping of the strong Raman peak 
originated from TAMRA at $1354 \mathrm{~cm}^{-1}$. SERS mapping is a method for generating detailed chemical images based on a sample's Raman spectrum. A complete spectrum is acquired at each and every pixel of the image in the selected area, and then interrogated to generate false color images based on intensity of a signature Raman peak. The SERS mapping of the distribution of TAMRA peak intensity at $1354 \mathrm{~cm}^{-1}$ over a $20 \mu \mathrm{m} \times 20 \mu \mathrm{m}$ selected area on peptide conjugated substrate was shown in red color in Figure 8.2(g). This mapping result confirms that peptide is distributed uniformly on the SERS substrate. In contrast, the TAMRA peak intensity is much weaker after incubating the substrate with MMP enzyme (Figure 8.2(h)), indicating that a majority of the peptide has been cleaved by the enzyme. This can be also indicated from SEM imaging in Figure 8.2(c) and (f), in which peptide layer disappears, indicating successful cleaving of peptide layer by enzyme. As a result, large amount of bound AuNPs on the nano-structures could be observed.

\subsubsection{Validation of the Mechanism}

To further validate the mechanism for enzyme detection, we perform a quantitative study by detecting individual MMP-2 or MMP-7 enzyme. The binding of AuNPs onto BMFON substrate occurs when the peptide on the substrate is cleaved by the corresponding enzyme, thus allowing binding on the biotin-avidin bond. By notion of the proportionality between the amount of AuNPs bound on BMFON substrate and peptide cleavage, the 
concentration of enzyme can be quantified by the intensity of target peaks of Raman tags. In this experiment, MMP-2 peptide- and MMP-7 peptide-conjugated BMFON were incubated with different concentrations of enzymes of MMP-2 and MMP-7, respectively. After peptide cleavage, NTand ATP-tagged AuNPs were then loaded on their corresponding substrates. The AuNPs bound substrates were then measured using Raman spectroscopy. Peaks at $1584 \mathrm{~cm}^{-1}$ and $1377 \mathrm{~cm}^{-1}$ were selected for intensity analysis as they are the most prominent and unhindered Raman peaks for ATP and NT, respectively (Figure 8.3(a) and (b)). We first investigated the SERS spectra of BMFON after bound with biotin-PEG-SH and biotin-avidin complex. As shown in Figure 8.3(c) and (d), the SERS intensities are weak and show no significant difference between BMFON with biotin and biotin-avidin complex. More importantly, the major peak at $1631 \mathrm{~cm}^{-1}$ is not interference with ATP or NT peaks. The SERS spectra after peptide cleavage and AuNPs binding were then investigated. Here, intensities of ATP and NT peaks increase with increasing enzyme concentrations, as shown in Figure 8.3(e) and (f). For BMFON incubated with control buffer containing no enzyme, the ATP or NT peak are negligible, indicating that the peptides have fully covered the substrates, thus preventing the binding of the AuNPs. The intensity curves of ATP and NT with enzyme concentrations are plotted in Figure 8.3(g) and (h). As tagged AuNPs bind onto substrates in a sandwich manner, thus greatly enhancing Raman intensities of ATP and NT. Therefore, the signal is 

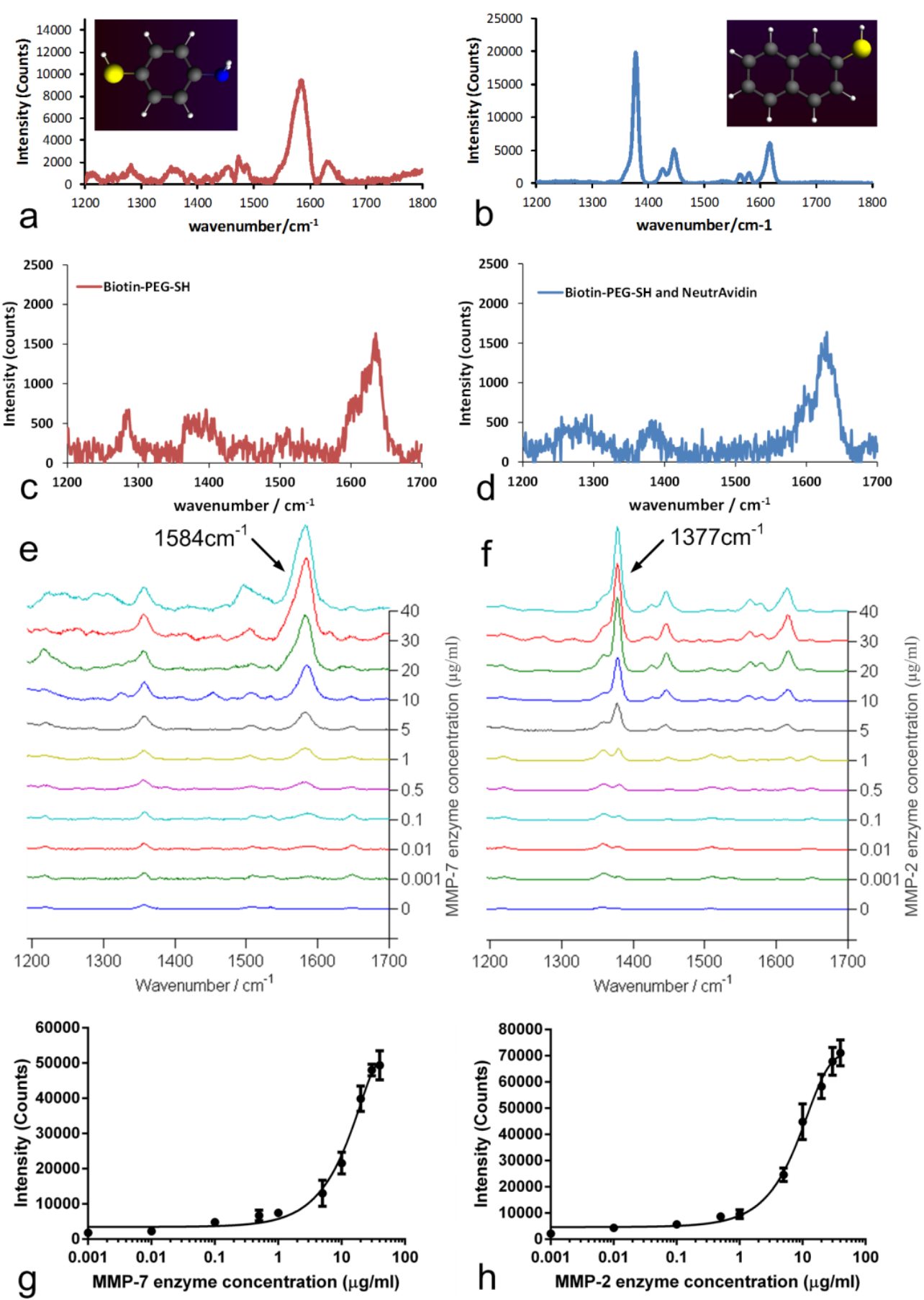

Figure 8.3 Detection of individual MMP enzymes. Chemical structure and

Raman spectra of (a) ATP and (b) NT. SERS spectra of (c) biotin-PEG-SH bound BMFON and (d) NeutrAvidin-biotin-PEG-SH bound BMFON. (e) SERS spectra of ATP tagged AuNPs bound MMP-7 conjugated BMFON after incubated with different concentrations of MMP-7 enzyme. (f) SERS spectra 
of NT tagged AuNPs bound MMP-2 conjugated BMFON after incubation with different concentrations of MMP-2 enzyme. (g) Intensity curve of the ATP Raman peak at $1584 \mathrm{~cm}^{-1}$ (x-axis is $\log 10$ scaled). (h) Intensity curve of the NT Raman peak at $1377 \mathrm{~cm}^{-1}$ (x-axis is $\log 10$ scaled).

detectable even in extremely low enzyme concentration. In the case of high enzyme concentration, a large majority of peptide is cleaved and AuNPs are fully bound to the substrate, causing SERS intensity of tagged AuNPs to saturate. The observations made above indicate that this platform is highly sensitive to MMP-2 and MMP-7 enzymes and can provide a wide detection range from $1 \mathrm{ng} / \mathrm{mL}$ to $40 \mu \mathrm{g} / \mathrm{mL}$. This detection range matches with expression level of MMP-2 and MMP-7 for many carcinomas at different stages. Gohji, K. et al reported of elevated serum MMP-2 level for superficial, advanced bladder carcinoma, and upper urothelial carcinoma are 550 \pm 142 , $696 \pm 213$ and $733 \pm 144 \mathrm{ng} / \mathrm{mL}$ [217]. While Andrea, P. et al, reported of serum MMP-7 level for colorectal tumors adenoma, stage I /II and III/ IV are 3.4, 4 and $5.5 \mathrm{ng} / \mathrm{mL}$ [203].

\subsubsection{Multiplexed Detection of MMP-2 and MMP-7 Enzymes}

To extend this platform for multiplexed detection of MMP-2 and MMP-7 enzymes, the BMFON substrates were conjugated with both MMP-2 and MMP-7 peptide whereas the NT- and ATP-tagged AuNPs were conjugated with MMP-2 peptide and MMP-7 peptide, respectively. In order to prove that the MMP peptides are able to fully shield the AuNPS, hydrodynamic 
diameters of the untagged AuNPs, tagged AuNPs and MMP shielded AuNPs were studied using DLS. The mean hydrodynamic diameter of untagged AuNPs was found to be $65.35 \mathrm{~nm}$, while the size increased to $69.00 \mathrm{~nm}$ after biotin conjugation and tagging with Raman reporter. After peptide shielding, the mean hydrodynamic diameter of MMP-7 conjugated AuNPs and MMP-2 conjugated AuNPs further increased to $71.47 \mathrm{~nm}$ and $73.14 \mathrm{~nm}$, indicating that a covering outer shell of MMP peptide has formed on AuNPs. In demonstrating the specificity in AuNP binding, four samples were prepared for detection: MMP-2 enzyme, MMP-7 enzyme, a mixture of MMP-2 and MMP-7 enzymes, and a control buffer containing no enzymes. As MMP enzyme cleaves only its designated peptide conjugated on the BMFON substrate and on its corresponding AuNP, allowing for specific labeling, multiplexed detection can be achieved from the unique Raman peaks and their intensities. As shown in Figure 8.4 (a), in the presence of control buffer, neither MMP-2 nor MMP-7 peptide is cleaved from BMFON substrate and AuNPs, resulting in no binding of AuNPs and, in turn, leading to negligible peak intensity at $1377 \mathrm{~cm}^{-1}$ and $1584 \mathrm{~cm}^{-1}$. In Figure 8.4(b), the MMP-2 enzyme cleaves MMP-2 peptides from BMFON and the NT-tagged AuNPs, allowing for sandwich formation between the substrate and AuNP, yielding a sharp $1377 \mathrm{~cm}^{-1}$ peak characteristic of NT. It is worthy to note that the 1584 $\mathrm{cm}^{-1}$ peak of ATP is negligible because no MMP-7 enzyme was present to cleave the MMP-7 peptide from BMFON and ATP-tagged AuNP for binding. 

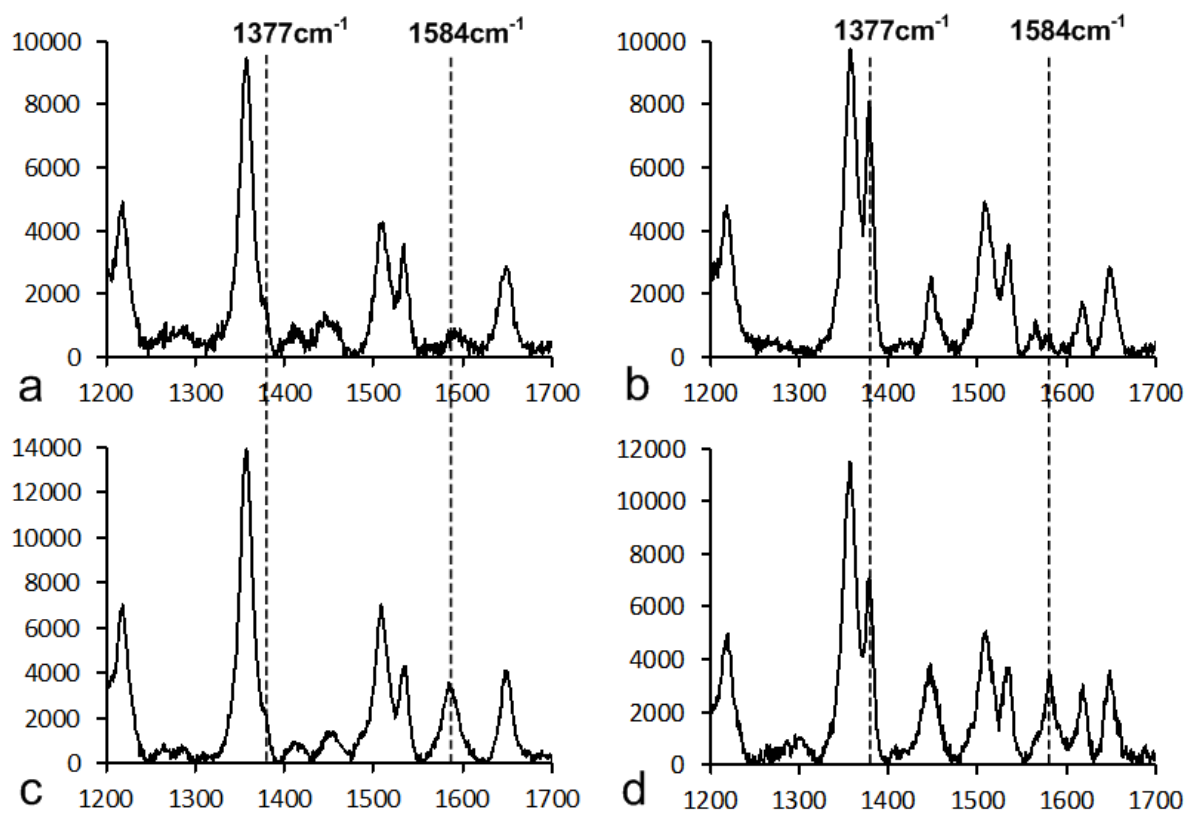

Figure 8.4 Multiplexed detection of MMP-2 and MMP-7 enzymes. Raman spectrum of MMP-2 and MMP-7 shielded BMFON substrate samples in the presence of (a) control solution contain no enzyme (b) MMP-2 enzyme (c) MMP-7 enzyme (d) both MMP-2 and MMP-7 enzyme.

Peak Assignment of Raman Spectra

\begin{tabular}{cl}
\hline Wavenumber $/ \mathrm{cm}^{-1}$ & \multicolumn{1}{c}{ Assignment } \\
\hline 1216 & N-N stretching, TAMRA \\
1354 & $\mathrm{CH}_{3}$ bend, TAMRA \\
1377 & CC ring stretching, NT \\
1445 & Ring stretch, NT \\
1510 & ring vibration, C=C in plane vibration, TAMRA \\
1534 & amide II, TAMRA \\
1584 & CC ring stretching, ATP \\
1615 & Ring stretch, NT \\
1631 & aromatic CC stretching vibrations, biotin \\
1646 & C=O and C=C stretching, TAMRA \\
\hline
\end{tabular}

Table 8.1 Table of peak Assignment of Raman Spectra [215, 216, 218]

Conversely in Figure 8.4(c), ATP peak at $1584 \mathrm{~cm}^{-1}$ could be observed while NT peak is negligible in the presence of MMP-7 enzyme. As in the case of the 
enzyme mixture, both NT and ATP peaks could be observed (Figure 8.4(d)). Intuitively, due to the presence of the two types of enzymes, both types of peptides were cleaved and AuNP binding to substrate could occur. In order to understand the Raman peaks, we tentatively assigned all major peaks observed in our study, as shown in Table 8.1.

\subsection{Summary}

We successfully demonstrated a SERS based platform for sensitive multiplexed detection of MMP-2 and MMP-7 proteinase using cleave-and-bind mechanism. To the best of our knowledge, this is the first reported SERS based MMP enzyme detection. This platform demonstrated a wide detection range from $1 \mathrm{ng} / \mathrm{mL}$ to $40 \mu \mathrm{g} / \mathrm{mL}$ for individual enzyme detection, thus it is able to detect the enzyme concentration from various clinical samples with different stages of cancers. In comparison to previously reported methods, our detection platform is simple and fast, providing readings from a much wider detection range with higher sensitivity. As a proof-of-concept, we successfully demonstrated the multiplexed detection for MMP-2 and MMP-7 enzymes as many cancer types have a close correlation to both enzyme expression levels, the multiplexed detection capability of this platform therefore provides a robust way to analysis these diseases. More importantly, this platform can be developed to clinical diagnostic tool to 
detect other MMP proteinase using the cleave-and-bind mechanism in bio-fluids samples. 


\section{Chapter 9 Conclusion and Future Works}

\subsection{Conclusions}

In this report, we discussed the synthesis, functionalization, characterization, and bio-photonic applications of AuNPs.

In the first section, we investigated the cytotoxicity, colloidal stability, and optical properties of AuNRs before and after functionalizing them with PEG-SH and Pluronic triblock copolymer (PEO-PPO-PEO) molecules. When using AuNRs for biological studies, it is important to eliminate or minimize the exposure of CTAB molecules from the AuNRs surface to the local environment such as cells or tissues. The complete removal of CTAB molecules from the AuNRs surface is unfeasible, as this will render the AuNRs structurally unstable, causing particle aggregation to occur. Here, we individually investigated the use of PEG-SH and PEO-PPO-PEO as capping agents to reduce the cytotoxicity of AuNR formulations while maintaining the optical, colloidal, and structural properties of AuNRs. We found that encapsulating AuNRs with PEG-SH or PEO-PPO-PEO molecules guarantees the stability and biocompatibility of the nano-formulation. However, excessive use of these molecules during the passivation process leads to a reduction in the overall cell viability. We also demonstrated the use of functionalized AuNRs as scattering probes for dark-field imaging of cancer 
cells, demonstrating their good biocompatibility. These results offer a unique solution for the future development of safe scattering color probes for clinical applications such as long-term imaging of cells and tissues.

In the second part of our work, the controlled synthesis of AuNRs and their toxicity concerns were reported and examined. More specifically, the encapsulation of AuNRs using Pluronic block copolymers was demonstrated. In comparison to CTAB stabilized AuNRs, UV-vis spectra indicated that the additional layering of Pluronic surfactants on the AuNRs surface did not change the shape or size of the particles. In addition, layering Pluronic surfactants on the particle surface was found to significantly improve the colloidal and optical stability of AuNRs formulations. To demonstrate the capability of Pluronic-coated AuNRs for in vitro imaging applications, cancer cells were treated with AuNRs formulations, directly imaged under dark-field imaging, and analyzed with a hyper-spectral system. The results of both the dark-field imaging and hyper-spectral analyses confirmed the uptake of Pluronic-encapsulated AuNRs by cancer cells. We envision that these engineered Pluronic-coated AuNRs formulations will open up new avenues for the development of real-time in vivo imaging probes for photo-thermal and photodynamic therapy.

In the third section, we showed that antibody-conjugated AuNSs and AuNRs can be used as label free plasmon scattering probes for multiplex dark-field imaging of cancer cells. Because AuNSs and AuNRs have their own unique 
SPR absorption spectra that can be tuned by modifying their shape and size, they can be easily identified using a hyper-spectral analysis system integrated with a dark-field microscopy imaging system. AuNSs formulations with an SPR peak at $537 \mathrm{~nm}$ and AuNRs formulations with longitudinal SPR peaks at $626 \mathrm{~nm}$ and $754 \mathrm{~nm}$ were prepared and conjugated with anti-EGFR antibodies for targeting and imaging OSCC cancer cell lines. From the hyper-spectral analysis, we were able to distinguish different types of AuNSs and AuNRs, even when they were internalized within cells. More importantly, in this work, we successfully demonstrated the concept of using AuNPs based plasmon scattering multiplexing probes for in vitro imaging applications. Based on the broadband white light excitation source and the tunability of AuNPs' longitudinal SPR range (ranging from 620 to $1300 \mathrm{~nm}$ ), we estimated that up to seven multiplex scattering probes with a wavelength resolution greater than $100 \mathrm{~nm}$ can be realized for specific molecular recognition.

Sandwiched SPR sensors formed by AuNPs and gold film have been reported to enhance the electrical field of the configuration, and thus increase the system sensitivity. However, to date, the effects of AuNR dimensions, the AuNR-to-film distance, and gold film thickness on SPR sensitivity remain poorly understood. In the fourth part of this work, we proposed an optimized sandwiched SPR bio-sensor for multiplex biomarker detection using gold sensing film coupled with AuNRs with different aspect ratios. The effects of AuNR dimensions, the AuNR-to-film distance, and gold film thickness on 
SPR signals were studied through FEM simulations. We optimized the film thickness and AuNR-to-film thickness to obtain the best sensing behavior. We also found that increasing the AuNR length resulted in a significant SPR red-shift, whereas the sensing signal responded much less to changes in AuNR width. Based on these results, we adopted three types of AuNRs with a length of 40,60 , or $80 \mathrm{~nm}$ and a width of $20 \mathrm{~nm}$ for the optimized sandwiched SPR enhanced sensing of multiplex IgG proteins. According to our simulation results, the bio-sensor was able to detect these proteins through different SPR absorption peaks corresponding to AuNRs with different aspect ratios. This concept shows great potential for the rapid, cost effective multiplex biomarker detection of many types of proteins.

To explore the application of AuNPs in SERS, we also developed a SERS-based fiber sensor to detect and quantify sialic acid on single cancer cell. This system adopted uniquely designed SC-PCF fiber together with 60 nm AuNPs to achieve strong “3D” SERS enhancement, and the DBA tag provided a non-interfered $2,000 \mathrm{~cm}^{-1}$ Raman peak. Given these advantages, this system was able to detect sialic acid on the single-cell level. We expect that this system can be further developed into a clinical diagnostic tool with broad applications in the highly sensitive detection of biomolecules from various types of liquid samples such as blood, serum, plasma, urine, saliva, sweat, and tears. 
In the last part of our work, we demonstrated a SERS-based assay for sensitive multiplexed detection of MMP-2 and MMP-7 proteinases. This assay adopted a cleave-and-bind mechanism by which the specific peptide chain was cleaved by its corresponding enzyme, resulting in the binding of corresponding tagged AuNPs. As a validation of the mechanism, the detection of individual enzymes was presented with a wide detection range, from 1 $\mathrm{ng} / \mathrm{mL}$ to $40 \mu \mathrm{g} / \mathrm{mL}$. Then, as a proof-of-concept, the multiplexed detection of MMP-2 and MMP-7 enzymes was successfully demonstrated. Considering that many cancer types are closely correlated to the expression of multiple enzymes, the multiplexed detection capability of this platform provides a robust way to analyze these diseases. More importantly, this cleave-and-bind mechanism can be used with many other types of enzymes, thus enabling its development as a clinical diagnostic tool for other diseases of interest.

\subsection{Future Works}

After a careful analysis of the work we have achieved so far, we recommend some additional future studies on the use of AuNPs for bio-photonic applications.

\subsubsection{Tunable AuNPs Enhanced SERS Platform}

SERS is crucial for biomedical detection because it can provide "finger print" signals for specific molecules. It is well known that the intensity of the Raman signal can be significantly enhanced with silver/gold nanoparticles, and thus 
one can use the SERS technique to detect specific biomolecules at the single-molecule level. Thus, SERS is suitable for sensing biomolecules at low concentrations. It is important for clinicians to detect cancer biomarkers such as HER2, p53, etc. during the early stages of cancer to improve patients' chances of survival. Therefore, it is essential to further develop AuNPs-based SERS bio-sensors with ultra-high sensitivity.

The nonlinear relationship between the enhancement factor and the local electromagnetic field strength is given approximately by the following equation:

$$
E F=\frac{\left|E_{l o c}\left(\omega_{I}\right)\right|^{2}\left|E_{l o c}\left(\omega_{R}\right)\right|^{2}}{\left|E_{\text {inc }}\right|^{4}}
$$

where $E_{l o c}$ is the local field at a point in the geometry, $E_{i n c}$ is the incident field strength, and $\omega_{I}$ and $\omega_{R}$ are the incident and Raman-scattered frequencies, respectively. To obtain the maximum $\mathrm{EF}$, it is important to enhance the electromagnetic field both at the incident frequency (laser excitation frequency) and Raman-scattered frequencies. Conventionally, the laser is excited at 532, 633, and $785 \mathrm{~nm}$, whereas the Raman-scattered frequencies of most molecules of interest are between $800-1800 \mathrm{~cm}^{-1}(556-914 \mathrm{~nm})$. The entire wavelength range falls in the tunable LSPR range of AuNPs; therefore, they can be adopted by SERS to match both the excitation and scattered wavelengths with their highly tunable LSPR peaks to achieve ultrahigh detection sensitivity. Thus, the SERS enhancement factor strongly depends on 
AuNPs. It is important to study the SERS enhancement factor of AuNPs with different shapes and sizes and of nanoparticles with different assemblages. FEM simulations and experimental testing should be performed to design and optimize the SERS platform, and this platform should be developed with both substrates and optical fibers for different applications. This proposed study will provide insight into the design of a SERS platform with ultrahigh sensitivity for bio-applications such as single-molecule detection. 


\section{List of Publications}

\section{Journal Publications}

[1] T. Gong, N. Zhang, K.V.Kong, D.Goh, G. Humbert, P.P Shum, et al., "Rapid SERS monitoring of lipid-peroxidation-derived protein modifications in cells using photonic crystal fiber sensor", Journal of biophotonics, Accepted.

[2] T. Gong, K.V.Kong, D.Goh, M. Olivo and K.-T. Yong, "Sensitive surface enhanced Raman scattering multiplexed detection of matrix metalloproteinase 2 and 7 cancer markers", Biomedical optical express, vol. 6, pp. 2076-2087, 2015.

[3] T. Gong, Y. Cui, D. Goh, K. V. Kong, P. P. Shum, G. Humbert, et al., "Highly sensitive SERS detection and quantification of sialic acid on single cell using photonic-crystal fiber with gold Nanoparticles," Biosensors and bioelectronics, vol. 64, pp. 227-233, 2015.

[4] T. Gong, D. Goh, M. Olivo, and K.-T. Yong, "In vitro toxicity and bioimaging studies of gold nanorods formulations coated with biofunctional thiol-PEG molecules and Pluronic block copolymers," Beilstein journal of nanotechnology, vol. 5, pp. 546-553, 2014.

[5] J. Zhu, T. Gong, A. Kopwitthaya, R. Hu, W.-C. Law, I. Roy, et al., "Synthesis of PEGylated gold nanorods (Au NRs) as absorption nanoprobes for near-infrared optical imaging," RSC Adv., vol. 3, pp. 12280-12286, 2013.

[6] T. Gong, M. Olivo, U. Dinish, D. Goh, K. V. Kong, and K.-T. Yong, "Engineering bioconjugated gold nanospheres and gold nanorods as label-free plasmon scattering probes for ultrasensitive multiplex dark-field imaging of cancer cells," Journal of biomedical nanotechnology, vol. 9, pp. 985-991, 2013.

[7] D. Goh†, T. Gong†, U. Dinish, K. K. Maiti, C. Y. Fu, K.-T. Yong, et al., "Pluronic triblock copolymer encapsulated gold nanorods as biocompatible localized plasmon resonance-enhanced scattering probes for dark-field imaging of cancer cells," Plasmonics, vol. 7, pp. 595-601, 2012. (†D. Goh and T. Gong contributed equally to this work.) 


\section{Conference Publications}

[1] T. Gong, U. Dinish, S. Zeng, M. Olivo, and K.-T. Yong, "Optimized sandwiched surface plasmon resonance enhanced biosensor for multiplex biomarker detection," in Photonics Global Conference (PGC), 2012, 2012, pp. 1 - 4. (Best poster award for Photonics Global Conference 2012) 


\section{References}

[1] K. L. Wustholz, A.-I. Henry, J. M. McMahon, R. G. Freeman, N. Valley, M. E. Piotti, et al., "Structure-Activity Relationships in Gold Nanoparticle Dimers and Trimers for Surface-Enhanced Raman Spectroscopy," Journal of the American Chemical Society, vol. 132, pp. 10903-10910, 2010/08/11 2010.

[2] L. Jing-Liang and M. Gu, "Gold-Nanoparticle-Enhanced Cancer Photothermal Therapy," Selected Topics in Quantum Electronics, IEEE Journal of, vol. 16, pp. 989-996, 2010.

[3] K. Eunjung, Y. Jaemoon, C. Jihye, S. Jin-Suck, H. Yong-Min, and H. Seungjoo, "Synthesis of gold nanorod-embedded polymeric nanoparticles by a nanoprecipitation method for use as photothermal agents," Nanotechnology, vol. 20, p. 365602, 2009.

[4] W. I. Choi, J.-Y. Kim, C. Kang, C. C. Byeon, Y. H. Kim, and G. Tae, "Tumor Regression In Vivo by Photothermal Therapy Based on Gold-Nanorod-Loaded, Functional Nanocarriers," ACS Nano, vol. 5, pp. 1995-2003, 2011/03/22 2011.

[5] M. E. Wieder, D. C. Hone, M. J. Cook, M. M. Handsley, J. Gavrilovic, and D. A. Russell, "Intracellular photodynamic therapy with photosensitizer-nanoparticle conjugates: cancer therapy using a "Trojan horse'," Photochemical \& Photobiological Sciences, vol. 5, pp. 727-734, 2006.

[6] S. Hsieh, S. Meltzer, C. R. C. Wang, A. A. G. Requicha, M. E. Thompson, and B. E. Koel, "Imaging and Manipulation of Gold Nanorods with an Atomic Force Microscope," The Journal of Physical Chemistry B, vol. 106, pp. 231-234, 2001.

[7] D. A. Schultz, "Plasmon resonant particles for biological detection," Current Opinion in Biotechnology, vol. 14, pp. 13-22, 2003.

[8] M. B. Mohamed, V. Volkov, S. Link, and M. A. El-Sayed, "The `lightning' gold nanorods: fluorescence enhancement of over a million compared to the gold metal," Chemical Physics Letters, vol. 317, pp. 517-523, 2000.

[9] B. Nikoobakht and M. A. El-Sayed, "Surface-Enhanced Raman Scattering Studies on Aggregated Gold Nanorods $\dagger$," The Journal of Physical Chemistry A, vol. 107, pp. 3372-3378, 2003/05/01 2003

[10] G. F. Paciotti, L. Myer, D. Weinreich, D. Goia, N. Pavel, R. E. McLaughlin, et al., "Colloidal Gold: A Novel Nanoparticle Vector for Tumor Directed Drug Delivery," Drug Delivery, vol. 11, pp. 169-183, 2004.

[11] M. Faraday, "The Bakerian Lecture: Experimental Relations of Gold (and Other Metals) to Light," Philosophical Transactions of the Royal Society of London, vol. 147, pp. 145-181, 1857.

[12] G. Mie, "Contributions to the optics of turbid media, particularly of colloidal metal solutions," Ann. Phys. , vol. 25, pp. 377-445, 1908.

[13] J. Turkevich, P. C. Stevenson, and J. Hillier, "A study of the nucleation and growth processes in the synthesis of colloidal gold," Discussions of the Faraday Society, vol. 11, pp. 55-75, 1951. 
[14] G. Frens, "Controlled Nucleation for the Regulation of the Particle Size in Monodisperse Gold Suspensions," Nature, vol. 241, pp. 20-22, 1973.

[15] M. Giersig and P. Mulvaney, "Preparation of ordered colloid monolayers by electrophoretic deposition," Langmuir, vol. 9, pp. 3408-3413, 1993/12/01 1993.

[16] M. Brust, M. Walker, D. Bethell, D. J. Schiffrin, and R. Whyman, "Synthesis of thiol-derivatised gold nanoparticles in a two-phase Liquid-Liquid system," Journal of the Chemical Society, Chemical Communications, vol. 0, pp. 801-802, 1994.

[17] N. R. Jana, L. Gearheart, and C. J. Murphy, "Seeding Growth for Size Control of 5-40 nm Diameter Gold Nanoparticles," Langmuir, vol. 17, pp. 6782-6786, 2001/10/01 2001 .

[18] B. Nikoobakht and M. A. El-Sayed, "Preparation and Growth Mechanism of Gold Nanorods (NRs) Using Seed-Mediated Growth Method," Chemistry of Materials, vol. 15, pp. 1957-1962, 2003.

[19] C. J. Johnson, E. Dujardin, S. A. Davis, C. J. Murphy, and S. Mann, "Growth and form of gold nanorods prepared by seed-mediated, surfactant-directed synthesis," Journal of Materials Chemistry, vol. 12, pp. 1765-1770, 2002.

[20] A. Gole and C. J. Murphy, "Seed-Mediated Synthesis of Gold Nanorods: Role of the Size and Nature of the Seed," Chemistry of Materials, vol. 16, pp. 3633-3640, 2004/09/01 2004

[21] B. D. Busbee, S. O. Obare, and C. J. Murphy, "An Improved Synthesis of High-Aspect-Ratio Gold Nanorods," Advanced Materials, vol. 15, pp. 414-416, 2003.

[22] F. Hao, C. L. Nehl, J. H. Hafner, and P. Nordlander, "Plasmon Resonances of a Gold Nanostar," Nano Letters, vol. 7, pp. 729-732, 2007/03/01 2007.

[23] S. Lal, S. E. Clare, and N. J. Halas, "Nanoshell-Enabled Photothermal Cancer Therapy: Impending Clinical Impact," Accounts of Chemical Research, vol. 41, pp. 1842-1851, 2008/12/16 2008.

[24] H.-L. Wu, C.-H. Kuo, and M. H. Huang, "Seed-Mediated Synthesis of Gold Nanocrystals with Systematic Shape Evolution from Cubic to Trisoctahedral and Rhombic Dodecahedral Structures," Langmuir, vol. 26, pp. 12307-12313, 2010/07/20 2010 .

[25] A. J. Haes, C. L. Haynes, A. D. McFarland, G. C. Schatz, R. P. Van Duyne, and S. Zou, "Plasmonic Materials for Surface-Enhanced Sensing and Spectroscopy," MRS Bulletin, vol. 30, pp. 368-375, 2005.

[26] K. L. Kelly, E. Coronado, L. L. Zhao, and G. C. Schatz, "The Optical Properties of Metal Nanoparticles: The Influence of Size, Shape, and Dielectric Environment," The Journal of Physical Chemistry B, vol. 107, pp. 668-677, 2003/01/01 2002.

[27] M. M. Miller and A. A. Lazarides, "Sensitivity of metal nanoparticle surface plasmon resonance to the dielectric environment," J Phys Chem B, vol. 109, pp. 21556-21565, 11/24 2005.

[28] J. J. Mock, M. Barbic, D. R. Smith, D. A. Schultz, and S. Schultz, "Shape effects in plasmon resonance of individual colloidal silver nanoparticles," The Journal of Chemical Physics, vol. 116, pp. 6755-6759, 04/15/ 2002. 
[29] C. L. Haynes, A. D. McFarland, M. T. Smith, J. C. Hulteen, and R. P. Van Duyne, "Angle-Resolved Nanosphere Lithography: Manipulation of Nanoparticle Size, Shape, and Interparticle Spacing," The Journal of Physical Chemistry B, vol. 106, pp. 1898-1902, 2002/02/01 2002.

[30] X. Huang, I. H. El-Sayed, W. Qian, and M. A. El-Sayed, "Cancer Cell Imaging and Photothermal Therapy in the Near-Infrared Region by Using Gold Nanorods," Journal of the American Chemical Society, vol. 128, pp. 2115-2120, 2006/02/01 2006.

[31] I. M. White and X. Fan, "On the performance quantification of resonant refractive index sensors," Optics Express, vol. 16, pp. 1020-1028, 2008/01/21 2008.

[32] D. W. Hahn, "Raman Scattering Theory," ed. Department of Mechanical and Aerospace Engineering, University of Florida, 2007.

[33] A. S. Haka, K. E. Shafer-Peltier, M. Fitzmaurice, J. Crowe, R. R. Dasari, and M. S. Feld, "Diagnosing breast cancer by using Raman spectroscopy," Proceedings of the National Academy of Sciences of the United States of America, vol. 102, pp. 12371-12376, August 30, 20052005.

[34] A. C. Ferrari, Basko, Denis M., "Raman spectroscopy as a versatile tool for studying the properties of graphene," Nat Nano, vol. 8, pp. 235-246, 2013.

[35] T. J. Römer, J. F. Brennan, M. Fitzmaurice, M. L. Feldstein, G. Deinum, J. L. Myles, et al., "Histopathology of Human Coronary Atherosclerosis by Quantifying Its Chemical Composition With Raman Spectroscopy," Circulation, vol. 97, pp. 878-885, March 10, 19981998.

[36] M. Fleischmann, P. J. Hendra, and A. J. McQuillan, "Raman spectra of pyridine adsorbed at a silver electrode," Chemical Physics Letters, vol. 26, pp. 163-166, 5/15/ 1974.

[37] D. L. Jeanmaire and R. P. Van Duyne, "Surface raman spectroelectrochemistry: Part I. Heterocyclic, aromatic, and aliphatic amines adsorbed on the anodized silver electrode," Journal of Electroanalytical Chemistry and Interfacial Electrochemistry, vol. 84, pp. 1-20, 11/10/ 1977.

[38] M. G. Albrecht and J. A. Creighton, "Anomalously intense Raman spectra of pyridine at a silver electrode," Journal of the American Chemical Society, vol. 99, pp. 5215-5217, 1977/06/01 1977.

[39] F. W. King, R. P. V. Duyne, and G. C. Schatz, "Theory of Raman scattering by molecules adsorbed on electrode surfaces," The Journal of Chemical Physics, vol. 69, pp. 4472-4481, 1978.

[40] S. Nie and S. R. Emory, "Probing Single Molecules and Single Nanoparticles by Surface-Enhanced Raman Scattering," Science, vol. 275, pp. 1102-1106, February 21, 19971997.

[41] E. Hao, G. Schatz, and J. Hupp, "Synthesis and Optical Properties of Anisotropic Metal Nanoparticles," Journal of Fluorescence, vol. 14, pp. 331-341, 2004/07/01 2004.

[42] G. von Maltzahn, A. Centrone, J.-H. Park, R. Ramanathan, M. J. Sailor, T. A. Hatton, et al., "SERS-Coded Gold Nanorods as a Multifunctional Platform for 
Densely Multiplexed Near-Infrared Imaging and Photothermal Heating," Advanced Materials, vol. 21, pp. 3175-3180, 2009.

[43] J. Song, J. Zhou, and H. Duan, "Self-Assembled Plasmonic Vesicles of SERS-Encoded Amphiphilic Gold Nanoparticles for Cancer Cell Targeting and Traceable Intracellular Drug Delivery," Journal of the American Chemical Society, vol. 134, pp. 13458-13469, 2012/08/15 2012.

[44] J. Ando, K. Fujita, N. I. Smith, and S. Kawata, "Dynamic SERS Imaging of Cellular Transport Pathways with Endocytosed Gold Nanoparticles," Nano Letters, vol. 11, pp. 5344-5348, 2011/12/14 2011.

[45] H. Xu, J. Aizpurua, M. Käll, and P. Apell, "Electromagnetic contributions to single-molecule sensitivity in surface-enhanced Raman scattering," Physical Review $E$, vol. 62, pp. 4318-4324, 09/01/ 2000.

[46] T. R. Jensen, G. C. Schatz, and R. P. Van Duyne, "Nanosphere Lithography: Surface Plasmon Resonance Spectrum of a Periodic Array of Silver Nanoparticles by Ultraviolet-Visible Extinction Spectroscopy and Electrodynamic Modeling," The Journal of Physical Chemistry B, vol. 103, pp. 2394-2401, 1999/04/01 1999.

[47] G. L. Liu and L. P. Lee, "Nanowell surface enhanced Raman scattering arrays fabricated by soft-lithography for label-free biomolecular detections in integrated microfluidics," Applied Physics Letters, vol. 87, p. 074101, 2005.

[48] F. J. García-Vidal and J. B. Pendry, "Collective Theory for Surface Enhanced Raman Scattering," Physical Review Letters, vol. 77, pp. 1163-1166, 08/05/ 1996.

[49] C. J. Orendorff, A. Gole, T. K. Sau, and C. J. Murphy, "Surface-Enhanced Raman Spectroscopy of Self-Assembled Monolayers: Sandwich Architecture and Nanoparticle Shape Dependence," Analytical Chemistry, vol. 77, pp. 3261-3266, 2005/05/01 2005.

[50] X. Hu, T. Wang, L. Wang, and S. Dong, "Surface-Enhanced Raman Scattering of 4-Aminothiophenol Self-Assembled Monolayers in Sandwich Structure with Nanoparticle Shape Dependence: Off-Surface Plasmon Resonance Condition," The Journal of Physical Chemistry C, vol. 111, pp. 6962-6969, 2007/05/01 2007.

[51] G. J. Kovacs, R. O. Loutfy, P. S. Vincett, C. Jennings, and R. Aroca, "Distance dependence of SERS enhancement factor from Langmuir-Blodgett monolayers on metal island films: evidence for the electromagnetic mechanism," Langmuir, vol. 2, pp. 689-694, 1986/11/01 1986.

[52] G. Chumanov, K. Sokolov, B. W. Gregory, and T. M. Cotton, "Colloidal metal films as a substrate for surface-enhanced spectroscopy," The Journal of Physical Chemistry, vol. 99, pp. 9466-9471, 1995/06/01 1995.

[53] E. Hao and G. C. Schatz, "Electromagnetic fields around silver nanoparticles and dimers," The Journal of Chemical Physics, vol. 120, pp. 357-366, 2004.

[54] Z. Chen, Z. Dai, N. Chen, S. Liu, F. Pang, B. Lu, et al., "Gold Nanoparticles-Modified Tapered Fiber Nanoprobe for Remote SERS Detection," IEEE Photonics Technology Letters, vol. 26, pp. 777-780, 2014.

[55] K. I. Mullen and K. T. Carron, "Surface-enhanced Raman spectroscopy with abrasively modified fiber optic probes," Analytical Chemistry, vol. 63, pp. 2196-2199, 1991/10/01 1991. 
[56] D. L. Stokes and T. Vo-Dinh, "Development of an integrated single-fiber SERS sensor," Sensors and Actuators B: Chemical, vol. 69, pp. 28-36, 9/10/ 2000.

[57] A. Lucotti and G. Zerbi, "Fiber-optic SERS sensor with optimized geometry," Sensors and Actuators B: Chemical, vol. 121, pp. 356-364, 2/20/ 2007.

[58] U. S. Dinish, G. Balasundaram, Y. T. Chang, and M. Olivo, "Sensitive multiplex detection of serological liver cancer biomarkers using SERS-active photonic crystal fiber probe," Journal of Biophotonics, 2013.

[59] H. Yan, C. Gu, C. Yang, J. Liu, G. Jin, J. Zhang, et al., "Hollow core photonic crystal fiber surface-enhanced Raman probe," Applied Physics Letters, vol. 89, p. 204101, 2006

[60] X. Yang, C. Shi, D. Wheeler, R. Newhouse, B. Chen, J. Z. Zhang, et al., "High-sensitivity molecular sensing using hollow-core photonic crystal fiber and surface-enhanced Raman scattering," Journal of the Optical Society of America A, vol. 27, pp. 977-984, 2010/05/01 2010.

[61] D. U. S, C. Y. Fu, K. S. Soh, B. Ramaswamy, A. Kumar, and M. Olivo, "Highly sensitive SERS detection of cancer proteins in low sample volume using hollow core photonic crystal fiber," Biosensors and Bioelectronics, vol. 33, pp. 293-298, 3/15/ 2012.

[62] Y. Zhang, C. Shi, C. Gu, L. Seballos, and J. Z. Zhang, "Liquid core photonic crystal fiber sensor based on surface enhanced Raman scattering," Applied Physics Letters, vol. 90, p. 193504, 2007.

[63] Knight and J. C., "Photonic crystal fibres," Nature, vol. 424, pp. 847-851, 2003.

[64] M. Skorobogatiy, "Microstructured and Photonic Bandgap Fibers for Applications in the Resonant Bio- and Chemical Sensors," Journal of Sensors, vol. 2009, 2009.

[65] C. J. Orendorff, T. K. Sau, and C. J. Murphy, "Shape-Dependent Plasmon-Resonant Gold Nanoparticles," Small, vol. 2, pp. 636-639, 2006.

[66] J. Zhou, J. Ralston, R. Sedev, and D. A. Beattie, "Functionalized gold nanoparticles: Synthesis, structure and colloid stability," Journal of Colloid and Interface Science, vol. 331, pp. 251-262, 3/15/ 2009.

[67] K. Sokolov, M. Follen, J. Aaron, I. Pavlova, A. Malpica, R. Lotan, et al., "Real-Time Vital Optical Imaging of Precancer Using Anti-Epidermal Growth Factor Receptor Antibodies Conjugated to Gold Nanoparticles," Cancer Research, vol. 63, pp. 1999-2004, May 1, 20032003.

[68] K. Sokolov, J. Aaron, B. Hsu, D. Nida, A. Gillenwater, M. Follen, et al., "Optical systems for in vivo molecular imaging of cancer," Technology in cancer research \& treatment, vol. 2, pp. 491-504, 12/ 2003.

[69] S. Eustis and M. El-Sayed, "Aspect Ratio Dependence of the Enhanced Fluorescence Intensity of Gold Nanorods: Experimental and Simulation Study," The Journal of Physical Chemistry B, vol. 109, pp. 16350-16356, 2005/09/01 2005.

[70] C.-Z. Li, K. B. Male, S. Hrapovic, and J. H. T. Luong, "Fluorescence properties of gold nanorods and their application for DNA biosensing," Chemical Communications, vol. 0, pp. 3924-3926, 2005.

[71] H. Wang, T. B. Huff, D. A. Zweifel, W. He, P. S. Low, A. Wei, et al., "In vitro and in vivo two-photon luminescence imaging of single gold nanorods," Proceedings of 
the National Academy of Sciences of the United States of America, vol. 102, pp. 15752-15756, November 1, 20052005.

[72] S. Link and M. A. El-Sayed, "Shape and size dependence of radiative, non-radiative and photothermal properties of gold nanocrystals," International Reviews in Physical Chemistry, vol. 19, pp. 409-453, 2000/07/01 2000.

[73] I. H. El-Sayed, X. Huang, and M. A. El-Sayed, "Selective laser photo-thermal therapy of epithelial carcinoma using anti-EGFR antibody conjugated gold nanoparticles," Cancer Letters, vol. 239, pp. 129-135, 7/28/ 2006.

[74] Y. C. Cao, R. Jin, and C. A. Mirkin, "Nanoparticles with Raman Spectroscopic Fingerprints for DNA and RNA Detection," Science, vol. 297, pp. 1536-1540, August 30, 20022002.

[75] X. Huang, I. H. El-Sayed, W. Qian, and M. A. El-Sayed, "Cancer Cells Assemble and Align Gold Nanorods Conjugated to Antibodies to Produce Highly Enhanced, Sharp, and Polarized Surface Raman Spectra: A Potential Cancer Diagnostic Marker," Nano Letters, vol. 7, pp. 1591-1597, 2007/06/01 2007.

[76] K. Kneipp, A. S. Haka, H. Kneipp, K. Badizadegan, N. Yoshizawa, C. Boone, et al., "Surface-Enhanced Raman Spectroscopy in Single Living Cells Using Gold Nanoparticles," Applied Spectroscopy, vol. 56, pp. 150-154, 2002/02/01 2002.

[77] X. Qian, X.-H. Peng, D. O. Ansari, Q. Yin-Goen, G. Z. Chen, D. M. Shin, et al., "In vivo tumor targeting and spectroscopic detection with surface-enhanced Raman nanoparticle tags," Nature biotechnology, vol. 26, pp. 83-90, 2008.

[78] J. Gao, C. M. Bender, and C. J. Murphy, "Dependence of the Gold Nanorod Aspect Ratio on the Nature of the Directing Surfactant in Aqueous Solution," Langmuir, vol. 19, pp. 9065-9070, 2003/10/01 2003.

[79] C. J. Murphy, A. M. Gole, J. W. Stone, P. N. Sisco, A. M. Alkilany, E. C. Goldsmith, et al., "Gold Nanoparticles in Biology: Beyond Toxicity to Cellular Imaging," Accounts of Chemical Research, vol. 41, pp. 1721-1730, 2008/12/16 2008.

[80] A. M. Alkilany, P. K. Nagaria, C. R. Hexel, T. J. Shaw, C. J. Murphy, and M. D. Wyatt, "Cellular Uptake and Cytotoxicity of Gold Nanorods: Molecular Origin of Cytotoxicity and Surface Effects," Small, vol. 5, pp. 701-708, 2009.

[81] A. K. Oyelere, P. C. Chen, X. Huang, I. H. El-Sayed, and M. A. El-Sayed, "Peptide-Conjugated Gold Nanorods for Nuclear Targeting," Bioconjugate Chemistry, vol. 18, pp. 1490-1497, 2007/09/01 2007.

[82] A. Agarwal, M. A. Mackey, M. A. El-Sayed, and R. V. Bellamkonda, "Remote Triggered Release of Doxorubicin in Tumors by Synergistic Application of Thermosensitive Liposomes and Gold Nanorods," ACS Nano, vol. 5, pp. 4919-4926, 2011/06/28 2011.

[83] J. D. Gibson, B. P. Khanal, and E. R. Zubarev, "Paclitaxel-Functionalized Gold Nanoparticles," Journal of the American Chemical Society, vol. 129, pp. 11653-11661, 2007/09/01 2007.

[84] T. Niidome, M. Yamagata, Y. Okamoto, Y. Akiyama, H. Takahashi, T. Kawano, et al., "PEG-modified gold nanorods with a stealth character for in vivo applications," Journal of Controlled Release, vol. 114, pp. 343-347, 9/12/ 2006. 
[85] H. N. Green, D. V. Martyshkin, C. M. Rodenburg, E. L. Rosenthal, and S. B. Mirov, "Gold Nanorod Bioconjugates for Active Tumor Targeting and Photothermal Therapy," Journal of Nanotechnology, vol. 2011, 2011.

[86] J. L. Li, D. Day, and M. Gu, "Ultra-Low Energy Threshold for Cancer Photothermal Therapy Using Transferrin-Conjugated Gold Nanorods," Advanced Materials, vol. 20, pp. 3866-3871, 2008.

[87] T. Gong, M. Olivo, U. S. Dinish, D. Goh, K. V. Kong, and K.-T. Yong, "Engineering Bioconjugated Gold Nanospheres and Gold Nanorods as Label-Free Plasmon Scattering Probes for Ultrasensitive Multiplex Dark-Field Imaging of Cancer Cells," Journal of Biomedical Nanotechnology, vol. 9, pp. 985-991, // 2013.

[88] L. H. Dubois and R. G. Nuzzo, "Synthesis, Structure, and Properties of Model Organic Surfaces," Annual Review of Physical Chemistry, vol. 43, pp. 437-463, 1992.

[89] P. Clarke. (2013). Reports: Globalfoundries hints at \$10-billion fab location.

[90] M. Iqbal, Y.-I. Chung, and G. Tae, "An enhanced synthesis of gold nanorods by the addition of Pluronic (F-127) via a seed mediated growth process," Journal of Materials Chemistry, vol. 17, pp. 335-342, 2007.

[91] D. Goh, T. Gong, U. S. Dinish, K. Maiti, C. Fu, K.-T. Yong, et al., "Pluronic Triblock Copolymer Encapsulated Gold Nanorods as Biocompatible Localized Plasmon Resonance-Enhanced Scattering Probes for Dark-Field Imaging of Cancer Cells," Plasmonics, vol. 7, pp. 595-601, 2012/12/01 2012.

[92] D. K. Smith and B. A. Korgel, "The Importance of the CTAB Surfactant on the Colloidal Seed-Mediated Synthesis of Gold Nanorods," Langmuir, vol. 24, pp. 644-649, 2008/02/01 2008.

[93] J. Pérez-Juste, I. Pastoriza-Santos, L. M. Liz-Marzán, and P. Mulvaney, "Gold nanorods: Synthesis, characterization and applications," Coordination Chemistry Reviews, vol. 249, pp. 1870-1901, 9// 2005.

[94] T. Sakai and P. Alexandridis, "Ag and Au Monometallic and Bimetallic Colloids: Morphogenesis in Amphiphilic Block Copolymer Solutions," Chemistry of Materials, vol. 18, pp. 2577-2583, 2006/05/01 2006.

[95] T. Sakai and P. Alexandridis, "Single-Step Synthesis and Stabilization of Metal Nanoparticles in Aqueous Pluronic Block Copolymer Solutions at Ambient Temperature," Langmuir, vol. 20, pp. 8426-8430, 2004/09/01 2004.

[96] C. J. van Oss, "Chapter Eight Stability Versus Flocculation of Aqueous Particle Suspensions," in Interface Science and Technology. vol. Volume 16, J. v. O. Carel, Ed., ed: Elsevier, 2008, pp. 113-130.

[97] S. Dunn, A. Brindley, S. Davis, M. Davies, and L. Illum, "Polystyrene-Poly (Ethylene Glycol) (PS-PEG2000) Particles as Model Systems for Site Specific Drug Delivery. 2. The Effect of PEG Surface Density on the in Vitro Cell Interaction and in VivoBiodistribution," Pharmaceutical Research, vol. 11, pp. 1016-1022, 1994/07/01 1994.

[98] S. Wang, W. Lu, O. Tovmachenko, U. S. Rai, H. Yu, and P. C. Ray, "Challenge in understanding size and shape dependent toxicity of gold nanomaterials in human skin keratinocytes," Chemical Physics Letters, vol. 463, pp. 145-149, 9/22/ 2008. 
[99] P. Sharma, S. Brown, G. Walter, S. Santra, and B. Moudgil, "Nanoparticles for bioimaging," Advances in Colloid and Interface Science, vol. 123-126, pp. 471-485, 11/16/ 2006.

[100] B. D. Chithrani, A. A. Ghazani, and W. C. W. Chan, "Determining the Size and Shape Dependence of Gold Nanoparticle Uptake into Mammalian Cells," Nano Letters, vol. 6, pp. 662-668, 2006/04/01 2006.

[101] A. Sanda CBS, "Detoxification of gold nanorods by conjugation with thiolated poly(ethylene glycol) and their assessment as SERS-active carriers of Raman tags," Nanotechnology, vol. 21, 2010.

[102] H. Takahashi, T. Niidome, T. Kawano, S. Yamada, and Y. Niidome, "Surface modification of gold nanorods using layer-by-layer technique for cellular uptake," Journal of Nanoparticle Research, vol. 10, pp. 221-228, 2008/01/01 2008.

[103] C. J. Murphy, L. B. Thompson, D. J. Chernak, J. A. Yang, S. T. Sivapalan, S. P. Boulos, et al., "Gold nanorod crystal growth: From seed-mediated synthesis to nanoscale sculpting," Current Opinion in Colloid \& Interface Science, vol. 16, pp. 128-134, 4// 2011.

[104] S. R. B. Sarwat F. Khattak, Susan C. Roberts, "Pluronic F127 as a cell encapsulation material: utilization of membrane-stabilizing agents," Tissue engineering, vol. 11, pp. 974-983, 2005.

[105] S. T. Reddy, A. Rehor, H. G. Schmoekel, J. A. Hubbell, and M. A. Swartz, "In vivo targeting of dendritic cells in lymph nodes with poly(propylene sulfide) nanoparticles," Journal of Controlled Release, vol. 112, pp. 26-34, 5/1/ 2006.

[106] J. J. Mock, D. R. Smith, and S. Schultz, "Local Refractive Index Dependence of Plasmon Resonance Spectra from Individual Nanoparticles," Nano Letters, vol. 3, pp. 485-491, 2003/04/01 2003.

[107] J. Yguerabide and E. E. Yguerabide, "Light-Scattering Submicroscopic Particles as Highly Fluorescent Analogs and Their Use as Tracer Labels in Clinical and Biological Applications: I. Theory," Analytical Biochemistry, vol. 262, pp. 137-156, 1998.

[108] J. Turkevich, G. Garton, and P. C. Stevenson, "The color of colloidal gold," Journal of Colloid Science, vol. 9, Supplement 1, pp. 26-35, 1954.

[109] O. Ehlert, R. Thomann, M. Darbandi, and T. Nann, "A Four-Color Colloidal Multiplexing Nanoparticle System," ACS Nano, vol. 2, pp. 120-124, 2008/01/01 2008.

[110] R. Hu, K.-T. Yong, I. Roy, H. Ding, S. He, and P. N. Prasad, "Metallic Nanostructures as Localized Plasmon Resonance Enhanced Scattering Probes for Multiplex Dark-Field Targeted Imaging of Cancer Cells," The Journal of Physical Chemistry C, vol. 113, pp. 2676-2684, 2009/02/19 2009.

[111] Z. Li, Y. Zhang, and S. Jiang, "Multicolor Core/Shell-Structured Upconversion Fluorescent Nanoparticles," Advanced Materials, vol. 20, pp. 4765-4769, 2008.

[112] C. L. Zavaleta, B. R. Smith, I. Walton, W. Doering, G. Davis, B. Shojaei, et al., "Multiplexed imaging of surface enhanced Raman scattering nanotags in living mice using noninvasive Raman spectroscopy," Proceedings of the National Academy of Sciences, vol. 106, pp. 13511-13516, August 11, 20092009. 
[113] K. K. Maiti, U. S. Dinish, A. Samanta, M. Vendrell, K.-S. Soh, S.-J. Park, et al., "Multiplex targeted in vivo cancer detection using sensitive near-infrared SERS nanotags," Nano Today, vol. 7, pp. 85-93, 2012.

[114] Y.-H. Chen, C.-Y. Tsai, P.-Y. Huang, M.-Y. Chang, P.-C. Cheng, C.-H. Chou, et al., "Methotrexate Conjugated to Gold Nanoparticles Inhibits Tumor Growth in a Syngeneic Lung Tumor Model," Molecular Pharmaceutics, vol. 4, pp. 713-722, 2007/10/01 2007.

[115] G. Burygin, B. Khlebtsov, A. Shantrokha, L. Dykman, V. Bogatyrev, and N. Khlebtsov, "On the Enhanced Antibacterial Activity of Antibiotics Mixed with Gold Nanoparticles," Nanoscale Research Letters, vol. 4, pp. 794 - 801, 2009.

[116] R. Bhattacharya, C. R. Patra, A. Earl, S. Wang, A. Katarya, L. Lu, et al., "Attaching folic acid on gold nanoparticles using noncovalent interaction via different polyethylene glycol backbones and targeting of cancer cells," Nanomedicine : nanotechnology, biology, and medicine, vol. 3, pp. 224-238, 2007.

[117] Y.-J. Gu, J. Cheng, C.-C. Lin, Y. W. Lam, S. H. Cheng, and W.-T. Wong, "Nuclear penetration of surface functionalized gold nanoparticles," Toxicology and Applied Pharmacology, vol. 237, pp. 196-204, 2009.

[118] G. Han, C. T. Martin, and V. M. Rotello, "Stability of Gold Nanoparticle-Bound DNA toward Biological, Physical, and Chemical Agents," Chemical Biology \& Drug Design, vol. 67, pp. 78-82, 2006.

[119] A. Wijaya, S. B. Schaffer, I. G. Pallares, and K. Hamad-Schifferli, "Selective Release of Multiple DNA Oligonucleotides from Gold Nanorods," ACS Nano, vol. 3 , pp. 80-86, 2009/01/27 2008.

[120] H. R. E. Kretschmann, "Radiative decay of nonradiative surface plasmons excited by light," Z. Naturforsch. A, vol. 23, 1968.

[121] S. Roh, T. Chung, and B. Lee, "Overview of the Characteristics of Micro- and Nano-Structured Surface Plasmon Resonance Sensors," Sensors, vol. 11, pp. 1565-1588, 2011.

[122] C. T. Campbell and G. Kim, "SPR microscopy and its applications to high-throughput analyses of biomolecular binding events and their kinetics," Biomaterials, vol. 28, pp. 2380-2392, 5// 2007.

[123] M. Piliarik, H. Vaisocherová, and J. Homola, "A new surface plasmon resonance sensor for high-throughput screening applications," Biosensors and Bioelectronics, vol. 20, pp. 2104-2110, 4/15/ 2005.

[124] J. Homola, "Surface plasmon resonance sensors for detection of chemical and biological species," Chemical reviews, vol. 108, p. 462, 2008.

[125] M. Piliarik, M. Vala, I. Tichý, and J. Homola, "Compact and low-cost biosensor based on novel approach to spectroscopy of surface plasmons," Biosensors and Bioelectronics, vol. 24, pp. 3430-3435, 8/15/ 2009.

[126] X. Fan, I. M. White, S. I. Shopova, H. Zhu, J. D. Suter, and Y. Sun, "Sensitive optical biosensors for unlabeled targets: A review," Analytica Chimica Acta, vol. 620, pp. 8-26, 7/14/ 2008. 
[127] L. A. Lyon, M. D. Musick, P. C. Smith, B. D. Reiss, D. J. Peña, and M. J. Natan, "Surface plasmon resonance of colloidal Au-modified gold films," Sensors and Actuators B: Chemical, vol. 54, pp. 118-124, 1999.

[128] L. A. Lyon, M. D. Musick, and M. J. Natan, "Colloidal Au-enhanced surface plasmon resonance immunosensing," Analytical Chemistry, vol. 70, pp. 5177-5183, 1998.

[129] X. Huang, H. Tu, D. Zhu, D. Du, and A. Zhang, "A gold nanoparticle labeling strategy for the sensitive kinetic assay of the carbamate-acetylcholinesterase interaction by surface plasmon resonance," Talanta, vol. 78, pp. 1036-1042, 2009.

[130] W.-C. Law, K.-T. Yong, A. Baev, and P. N. Prasad, "Sensitivity improved surface plasmon resonance biosensor for cancer biomarker detection based on plasmonic enhancement," ACS nano, vol. 5, pp. 4858-4864, 2011.

[131] J. H. a. M. Piliarik, Surface Plasmon Resonance Based Sensors vol. 4, 2006.

[132] G. G. Nenninger, M. Piliarik, and J. Homola, "Data analysis for optical sensors based on spectroscopy of surface plasmons," Measurement Science and Technology, vol. 13, p. 2038, 2002.

[133] L. Gou and C. J. Murphy, "Fine-Tuning the Shape of Gold Nanorods," Chemistry of Materials, vol. 17, pp. 3668-3672, 2005/07/01 2005.

[134] J. S. Mitchell, Y. Wu, C. J. Cook, and L. Main, "Sensitivity enhancement of surface plasmon resonance biosensing of small molecules," Analytical Biochemistry, vol. 343, pp. 125-135, 8/1/ 2005.

[135] A. Varki, "Glycan-based interactions involving vertebrate sialic-acid-recognizing proteins," Nature, vol. 446, p. 7, 2007.

[136] M. Li, L. Song, and X. Qin, "Glycan changes: cancer metastasis and anti-cancer vaccines," Journal of Biosciences, vol. 35, pp. 665-673, 2010/12/01 2010.

[137] A. Cazet, S. Julien, M. Bobowski, J. Burchell, and P. Delannoy, "Tumour-associated carbohydrate antigens in breast cancer," Breast Cancer Research, vol. 12, p. 204, 2010.

[138] Y.-Y. Zhao, M. Takahashi, J.-G. Gu, E. Miyoshi, A. Matsumoto, S. Kitazume, et al., "Functional roles of $\mathrm{N}$-glycans in cell signaling and cell adhesion in cancer," Cancer Science, vol. 99, pp. 1304-1310, 2008.

[139] S. N. CHARI and N. NATH, "Sialic Acid Content and Sialidase Activity of Polymorphonuclear Leucocytes in Diabetes Mellitus," The American Journal of the Medical Sciences, vol. 288, pp. 18-20, 1984.

[140] M. M. E. Fuster, Jeffrey D., "The sweet and sour of cancer: glycans as novel therapeutic targets," Nat Rev Cancer, vol. 5, pp. 526-542, 2005.

[141] C. Warren, "Leonard. The thiobarbituric acid assay of sialic acids," Journal of biological chemistry, vol. 234, pp. 1971-5, 1959.

[142] L. Svennerholm, "Quantitive estimation of sialic acids: II. A colorimetric resorcinol-hydrochloric acid method," Biochimica et Biophysica Acta, vol. 24, pp. 604-611, // 1957.

[143] N. Katopodis, Y. Hirshaut, N. L. Geller, and C. C. Stock, "Lipid-associated Sialic Acid Test for the Detection of Human Cancer," Cancer Research, vol. 42, pp. 5270-5275, December 1, 19821982. 
[144] K. R. Anumula, "Rapid Quantitative Determination of Sialic Acids in Glycoproteins by High-Performance Liquid Chromatography with a Sensitive Fluorescence Detection," Analytical Biochemistry, vol. 230, pp. 24-30, 9// 1995.

[145] T. Kawai, A. Kato, H. Higashi, S. Kato, and M. Naiki, "Quantitative Determination of N-Glycolylneuraminic Acid Expression in Human Cancerous Tissues and Avian Lymphoma Cell Lines as a Tumor-associated Sialic Acid by Gas Chromatography-Mass Spectrometry," Cancer Research, vol. 51, pp. 1242-1246, February 15, 19911991.

[146] A. E. Manzi, S. Diaz, and A. Varki, "High-pressure liquid chromatography of sialic acids on a pellicular resin anion-exchange column with pulsed amperometric detection: A comparison with six other systems," Analytical Biochemistry, vol. 188, pp. 20-32, 7// 1990.

[147] A. Matsumoto, N. Sato, K. Kataoka, and Y. Miyahara, "Noninvasive Sialic Acid Detection at Cell Membrane by Using Phenylboronic Acid Modified Self-Assembled Monolayer Gold Electrode," Journal of the American Chemical Society, vol. 131, pp. 12022-12023, 2009/09/02 2009.

[148] J. E. Freebersyser, M. T. Drake, L. K. Riley, M. H. Myles, and R. S. Livingston, "Evaluation of a commercial colorimetric fecal dipstick assay for the detection of Helicobacter hepaticus infections in laboratory mice," Journal of the American Association for Laboratory Animal Science, vol. 49, pp. 312-315, // 2010.

[149] K. König, "Multiphoton microscopy in life sciences," Journal of Microscopy, vol. 200, pp. 83-104, 2000.

[150] W. R. Zipfel, R. M. Williams, R. Christie, A. Y. Nikitin, B. T. Hyman, and W. W. Webb, "Live tissue intrinsic emission microscopy using multiphoton-excited native fluorescence and second harmonic generation," Proceedings of the National Academy of Sciences, vol. 100, pp. 7075-7080, June 10, 20032003.

[151] J. Widengren and R. Rigler, "Mechanisms of photobleaching investigated by fluorescence correlation spectroscopy," Bioimaging, vol. 4, pp. 149-157, 1996.

[152] P. K. Ray and R. L. Simmons, "Differential release of sialic acid from normal and malignant cells by Vibrio cholerae neuraminidase or influenza virus neuraminidase," Cancer research, vol. 33, pp. 936-939, 1973.

[153] S. J. Stray, R. D. Cummings, and G. M. Air, "Influenza virus infection of desialylated cells," Glycobiology, vol. 10, pp. 649-658, July 1, 20002000.

[154] S. P. Galuska, H. Geyer, B. Weinhold, M. Kontou, R. C. Röhrich, U. Bernard, et al., "Quantification of Nucleotide-Activated Sialic Acids by a Combination of Reduction and Fluorescent Labeling," Analytical Chemistry, vol. 82, pp. 4591-4598, 2010/06/01 2010.

[155] J. Kneipp, H. Kneipp, and K. Kneipp, "SERS-a single-molecule and nanoscale tool for bioanalytics," Chemical Society Reviews, vol. 37, pp. 1052-1060, 2008.

[156] E. C. Le Ru, M. Meyer, and P. G. Etchegoin, "Proof of Single-Molecule Sensitivity in Surface Enhanced Raman Scattering (SERS) by Means of a Two-Analyte Technique," The Journal of Physical Chemistry B, vol. 110, pp. 1944-1948, 2006/02/01 2006 
[157] A. Campion and P. Kambhampati, "Surface-enhanced Raman scattering," Chemical Society Reviews, vol. 27, pp. 241-250, 1998.

[158] S. S. R. Dasary, A. K. Singh, D. Senapati, H. Yu, and P. C. Ray, "Gold Nanoparticle Based Label-Free SERS Probe for Ultrasensitive and Selective Detection of Trinitrotoluene," Journal of the American Chemical Society, vol. 131, pp. 13806-13812, 2009/09/302009.

[159] Z. Xie, J. Tao, Y. Lu, K. Lin, J. Yan, P. Wang, et al., "Polymer optical fiber SERS sensor with gold nanorods," Optics Communications, vol. 282, pp. 439-442, 2/1/ 2009.

[160] J. Scaffidi, M. Gregas, V. Seewaldt, and T. Vo-Dinh, "SERS-based plasmonic nanobiosensing in single living cells," Analytical and Bioanalytical Chemistry, vol. 393, pp. 1135-1141, 2009/02/01 2009.

[161] J. D. Love and W. M. Henry, "Quantifying loss minimisation in single-mode fibre tapers," Electronics Letters, vol. 22, pp. 912-914, 1986.

[162] H. Yan, C. Gu, C. Yang, J. Liu, G. Jin, J. Zhang, et al., "Hollow core photonic crystal fiber surface-enhanced Raman probe," Applied Physics Letters, vol. 89, pp. -, 2006.

[163] J. De Gelder, K. De Gussem, P. Vandenabeele, and L. Moens, "Reference database of Raman spectra of biological molecules," Journal of Raman Spectroscopy, vol. 38, pp. 1133-1147, 2007.

[164] G. J. Puppels, F. F. M. de Mul, C. G. Otto, J., M. Robert-Nicoud, D. J. Arndt-Jovin, and T. M. Jovin, "Studying single living cells and chromosomes by confocal Raman microspectroscopy," Nature, vol. 347, pp. 301-303, 1990.

[165] D. C. Kennedy, C. S. McKay, L.-l. Tay, Y. Rouleau, and J. P. Pezacki, "Carbon-bonded silver nanoparticles: alkyne-functionalized ligands for SERS imaging of mammalian cells," Chemical Communications, vol. 47, pp. 3156-3158, 2011.

[166] L. J. Bellamy, "Alkynes and Allenes," in The Infra-red Spectra of Complex Molecules, ed: Springer Netherlands, 1975, pp. 64-71.

[167] H. Otsuka, E. Uchimura, H. Koshino, T. Okano, and K. Kataoka, "Anomalous Binding Profile of Phenylboronic Acid with N-Acetylneuraminic Acid (Neu5Ac) in Aqueous Solution with Varying pH," Journal of the American Chemical Society, vol. 125, pp. 3493-3502, 2003/03/01 2003.

[168] A. Liu, S. Peng, J. C. Soo, M. Kuang, P. Chen, and H. Duan, "Quantum Dots with Phenylboronic Acid Tags for Specific Labeling of Sialic Acids on Living Cells," Analytical Chemistry, vol. 83, pp. 1124-1130, 2011/02/01 2010.

[169] M. Mareel, L. Ridder, and J. Deman, "Histochemical study of the distribution of sialic acid on the surface of HeLa cells in culture," Histochemie, vol. 32, pp. 335-341, 1972/12/01 1972.

[170] D. W. Tan, K. B. Jensen, M. W. Trotter, J. T. Connelly, S. Broad, and F. M. Watt, "Single-cell gene expression profiling reveals functional heterogeneity of undifferentiated human epidermal cells," Development, vol. 140, pp. 1433-1444, 2013. 
[171] E. D. Palik, "Handbook of Optical Constants of Solids." vol. I - III E. D. Palik, Ed., ed Burlington: Academic Press, 1997.

[172] N. Felidj, J. Aubard, G. Levi, J. R. Krenn, A. Hohenau, G. Schider, et al., "Optimized surface-enhanced Raman scattering on gold nanoparticle arrays," Applied Physics Letters, vol. 82, pp. 3095-3097, 05/05/ 2003.

[173] Y. Cui, G. Humbert, Q. X. Dinh, F. Luan, N. H. Vu, T. Q. Tien, et al., "Design and fabrication of side-channel photonic crystal fiber," in Photonics Global Conference (PGC), 2012, 2012, pp. 1-3.

[174] K. Kneipp, H. Kneipp, I. Itzkan, R. R. Dasari, and M. S. Feld, "Ultrasensitive Chemical Analysis by Raman Spectroscopy," Chemical Reviews, vol. 99, pp. 2957-2976, 1999/10/01 1999.

[175] J. Fontana, J. Livenere, F. J. Bezares, J. D. Caldwell, R. Rendell, and B. R. Ratna, "Large surface-enhanced Raman scattering from self-assembled gold nanosphere monolayers," Applied Physics Letters, vol. 102, pp. 201606-201606-5, 2013.

[176] V. Joseph, A. Matschulat, J. Polte, S. Rolf, F. Emmerling, and J. Kneipp, "SERS enhancement of gold nanospheres of defined size," Journal of Raman Spectroscopy, vol. 42, pp. 1736-1742, 2011.

[177] A. K. Sinha and G. Melnykovych, "Sialic acid in prednisolone treated Hela cells; suggested mechanism for elevated levels at the cell surface," Biochemical and Biophysical Research Communications, vol. 49, pp. 984-991, 11/15/ 1972.

[178] L. Warren, "The Thiobarbituric Acid Assay of Sialic Acids," Journal of Biological Chemistry, vol. 234, pp. 1971-1975, August 1, 19591959.

[179] J. A. Key, C. Li, and C. W. Cairo, "Detection of Cellular Sialic Acid Content Using Nitrobenzoxadiazole Carbonyl-Reactive Chromophores," Bioconjugate Chemistry, vol. 23, pp. 363-371, 2012/03/21 2012.

[180] Y. Zhou, H. Dong, L. Liu, J. Liu, and M. Xu, "A novel potentiometric sensor based on a poly (anilineboronic acid)/graphene modified electrode for probing sialic acid through boronic acid-diol recognition," Biosensors and Bioelectronics, vol. 60, pp. 231-236, 2014.

[181] H. Sato, T. Takino, Y. Okada, J. Cao, A. Shinagawa, E. Yamamoto, et al., "A matrix metalloproteinase expressed on the surface of invasive tumour cells," Nature, vol. 370, pp. 61-65, 1994.

[182] C. Gialeli, A. D. Theocharis, and N. K. Karamanos, "Roles of matrix metalloproteinases in cancer progression and their pharmacological targeting," $\mathrm{FeBS}$ Journal, vol. 278, pp. 16-27, 2011.

[183] B. Schmalfeldt, D. Prechtel, K. Härting, K. Späthe, S. Rutke, E. Konik, et al., "Increased expression of matrix metalloproteinases (MMP)-2, MMP-9, and the urokinase-type plasminogen activator is associated with progression from benign to advanced ovarian cancer," Clinical Cancer Research, vol. 7, pp. 2396-2404, 2001.

[184] H. A. Kenny and E. Lengyel, "MMP-2 functions as an early response protein in ovarian cancer metastasis," Cell cycle (Georgetown, Tex.), vol. 8, pp. 683-688, 03/25 2009.

[185] B. Davidson, I. Goldberg, W. Gotlieb, J. Kopolovic, G. Ben-Baruch, J. Nesland, et al., "High levels of MMP-2, MMP-9, MT1-MMP and TIMP-2 mRNA correlate with 
poor survival in ovarian carcinoma," Clinical \& Experimental Metastasis, vol. 17, pp. 799-808, 1999/10/01 1999.

[186] A. Köhrmann, U. Kammerer, M. Kapp, J. Dietl, and J. Anacker, "Expression of matrix metalloproteinases (MMPs) in primary human breast cancer and breast cancer cell lines: New findings and review of the literature," BMC cancer, vol. 9, p. 188, 2009.

[187] H. J. Zhang, W. Zhao, S. Venkataraman, M. E. Robbins, G. R. Buettner, K. C. Kregel, et al., "Activation of matrix metalloproteinase-2 by overexpression of manganese superoxide dismutase in human breast cancer MCF-7 cells involves reactive oxygen species," Journal of Biological Chemistry, vol. 277, pp. 20919-20926, 2002.

[188] C. Gilles, J. A. Bassuk, H. Pulyaeva, E. H. Sage, J.-M. Foidart, and E. W. Thompson, "SPARC/osteonectin induces matrix metalloproteinase 2 activation in human breast cancer cell lines," Cancer Research, vol. 58, pp. 5529-5536, 1998.

[189] K. Gohji, N. Fujimoto, T. Komiyama, A. Fujii, J. Ohkawa, S. Kamidono, et al., "Elevation of serum levels of matrix metalloproteinase - 2 and - 3 as new predictors of recurrence in patients with urothelial carcinoma," Cancer, vol. 78, pp. 2379-2387, 1996.

[190] K. Kanda, M. Takahashi, Y. Murakami, H. Kanayama, and S. Kagawa, "The role of the activated form of matrix metalloproteinase-2 in urothelial cancer," $B J U$ International, vol. 86, pp. 553-557, 2000.

[191] K. Ohashi, T. Nemoto, K. Nakamura, and R. Nemori, "Increased expression of matrix metalloproteinase 7 and 9 and membrane type 1 - matrix metalloproteinase in esophageal squamous cell carcinomas," Cancer, vol. 88, pp. 2201-2209, 2000.

[192] Y. Tanioka, T. Yoshida, T. Yagawa, Y. Saiki, S. Takeo, T. Harada, et al., "Matrix metalloproteinase-7 and matrix metalloproteinase-9 are associated with unfavourable prognosis in superficial oesophageal cancer," British journal of cancer, vol. 89, pp. 2116-2121, 2003

[193] K. Yamashita, M. Mori, T. Shiraishi, K. Shibuta, and K. Sugimachi, "Clinical Significance of Matrix Metalloproteinase-7 Expression in Esophageal Carcinoma," Clinical Cancer Research, vol. 6, pp. 1169-1174, March 1, 20002000.

[194] H. Sasaki, H. Yukiue, S. Moiriyama, Y. Kobayashi, Y. Nakashima, M. Kaji, et al., "Clinical significance of matrix metalloproteinase-7 and Ets-1 gene expression in patients with lung cancer," Journal of Surgical Research, vol. 101, pp. 242-247, 2001.

[195] B. Passlick, W. Sienel, R. Seen-Hibler, W. Wöckel, O. Thetter, W. Mutschler, et al., "Overexpression of matrix metalloproteinase 2 predicts unfavorable outcome in early-stage non-small cell lung cancer," Clinical Cancer Research, vol. 6, pp. 3944-3948, 2000.

[196] C.-B. Guo, S. Wang, C. Deng, D.-L. Zhang, F.-L. Wang, and X.-Q. Jin, "Relationship between Matrix Metalloproteinase 2 and Lung Cancer Progression," Molecular Diagnosis \& Therapy, vol. 11, pp. 183-192, 2007/05/01 2007.

[197] L. E. Jones, M. J. Humphreys, F. Campbell, J. P. Neoptolemos, and M. T. Boyd, "Comprehensive Analysis of Matrix Metalloproteinase and Tissue Inhibitor 
Expression in Pancreatic Cancer: Increased Expression of Matrix Metalloproteinase-7 Predicts Poor Survival," Clinical Cancer Research, vol. 10, pp. 2832-2845, April 15, 20042004.

[198] V. Ellenrieder, B. Alber, U. Lacher, S. F. Hendler, A. Menke, W. Boeck, et al., "Role of MT-MMPs and MMP-2 in pancreatic cancer progression," International Journal of Cancer, vol. 85, pp. 14-20, 2000.

[199] S. McDonnell, M. Navre, R. J. Coffey, and L. M. Matrisian, "Expression and localization of the matrix metalloproteinase pump-1 (MMP-7) in human gastric and colon carcinomas," Molecular Carcinogenesis, vol. 4, pp. 527-533, 1991.

[200] C. F. Sier, F. J. Kubben, S. Ganesh, M. M. Heerding, G. Griffioen, R. Hanemaaijer, et al., "Tissue levels of matrix metalloproteinases MMP-2 and MMP-9 are related to the overall survival of patients with gastric carcinoma," British Journal of Cancer, vol. 74, pp. 413-417, 1996.

[201] M. Hilska, P. J. Roberts, Y. U. Collan, V. J. O. Laine, J. Kössi, P. Hirsimäki, et al., "Prognostic significance of matrix metalloproteinases - 1, - 2, - 7 and - 13 and tissue inhibitors of metalloproteinases - 1, - 2,-3 and - 4 in colorectal cancer," International journal of cancer, vol. 121, pp. 714-723, 2007.

[202] M. Pesta, L. Holubec, O. Topolcan, M. Cerna, K. Rupert, L. H. SEN, et al. "Quantitative estimation of matrix metalloproteinases 2 and 7 (MMP-2, MMP-7) and tissue inhibitors of matrix metalloproteinases 1 and 2 (TIMP-1, TIMP-2) in colorectal carcinoma tissue samples," Anticancer research, vol. 25, pp. 3387-3391, 2005.

[203] A. Polistena, A. Cucina, S. Dinicola, C. Stene, G. Cavallaro, A. Ciardi, et al., "MMP7 expression in colorectal tumours of different stages," in vivo, vol. 28, pp. 105-110, 2014.

[204] G. La Rocca, I. Pucci-Minafra, A. Marrazzo, P. Taormina, and S. Minafra, "Zymographic detection and clinical correlations of MMP-2 and MMP-9 in breast cancer sera," British journal of cancer, vol. 90, pp. 1414-1421, 2004.

[205] H. Frankowski, Y.-H. Gu, J. H. Heo, R. Milner, and G. J. del Zoppo, "Use of gel zymography to examine matrix metalloproteinase (gelatinase) expression in brain tissue or in primary glial cultures," in Astrocytes, ed: Springer, 2012, pp. 221-233

[206] S. Eissa, R. Ali-Labib, M. Swellam, M. Bassiony, F. Tash, and T. M. El-Zayat, "Noninvasive Diagnosis of Bladder Cancer by Detection of Matrix Metalloproteinases (MMP-2 and MMP-9) and Their Inhibitor (TIMP-2) in Urine," European Urology, vol. 52, pp. 1388-1397, 11// 2007.

[207] L. Shi, V. De Paoli, N. Rosenzweig, and Z. Rosenzweig, "Synthesis and Application of Quantum Dots FRET-Based Protease Sensors," Journal of the American Chemical Society, vol. 128, pp. 10378-10379, 2006/08/01 2006

[208] P. Chen, R. Selegard, D. Aili, and B. Liedberg, "Peptide functionalized gold nanoparticles for colorimetric detection of matrilysin (MMP-7) activity," Nanoscale, vol. 5, pp. 8973-8976, 2013.

[209] K. Jung, A. Ramankulov, M. Schrader, K. Miller, and M. Lein, "Circulating matrix metalloproteinase-7: an early or metastatic marker for renal cell carcinoma?," Clinical chemistry, vol. 54, pp. 1927-1929, 2008. 
[210] M. G. Tutton, M. L. George, S. A. Eccles, S. Burton, R. I. Swift, and A. M. Abulafi, "Use of plasma MMP-2 and MMP-9 levels as a surrogate for tumour expression in colorectal cancer patients," International Journal of Cancer, vol. 107, pp. 541-550, 2003.

[211] C. Y. Fu, K. W. Kho, U. S. Dinish, Z. Y. Koh, and O. Malini, "Enhancement in SERS intensity with hierarchical nanostructures by bimetallic deposition approach," Journal of Raman Spectroscopy, vol. 43, pp. 977-985, 2012.

[212] J. L. Seltzer, K. T. Akers, H. Weingarten, G. A. Grant, D. W. McCourt, and A. Z. Eisen, "Cleavage specificity of human skin type IV collagenase (gelatinase). Identification of cleavage sites in type I gelatin, with confirmation using synthetic peptides," Journal of Biological Chemistry, vol. 265, pp. 20409-13, November 25, 19901990.

[213] B. E. Turk, L. L. Huang, E. T. Piro, and L. C. Cantley, "Determination of protease cleavage site motifs using mixture-based oriented peptide libraries," Nature biotechnology, vol. 19, pp. 661-667, 2001.

[214] Y. Xu, Q. Cao, F. Svec, and J. M. J. Fréchet, "Porous Polymer Monolithic Column with Surface-Bound Gold Nanoparticles for the Capture and Separation of Cysteine-Containing Peptides," Analytical Chemistry, vol. 82, pp. 3352-3358, 2010/04/15 2010 .

[215] J. Zheng, Y. Zhou, X. Li, Y. Ji, T. Lu, and R. Gu, "Surface-Enhanced Raman Scattering of 4-Aminothiophenol in Assemblies of Nanosized Particles and the Macroscopic Surface of Silver," Langmuir, vol. 19, pp. 632-636, 2003/02/01 2003.

[216] R. A. Alvarez-Puebla, D. S. Dos Santos Jr, and R. F. Aroca, "Surface-enhanced Raman scattering for ultrasensitive chemical analysis of 1 and 2-naphthalenethiols," Analyst, vol. 129, pp. 1251-1256, 2004.

[217] K. Gohji, N. Fujimoto, T. Komiyama, A. Fujii, J. Ohkawa, S. Kamidono, et al., "Elevation of serum levels of matrix metalloproteinase-2 and -3 as new predictors of recurrence in patients with urothelial carcinoma," Cancer, vol. 78, pp. 2379-2387, 1996.

[218] P. Negri, R. J. Flaherty, O. O. Dada, and Z. D. Schultz, "Ultrasensitive online SERS detection of structural isomers separated by capillary zone electrophoresis," Chemical Communications, vol. 50, pp. 2707-2710, 2014. 